\title{
KAM theorem with normal frequencies of finite limit-points for some shallow water equations*
}

\author{
Xiaoping Yuan ${ }^{\dagger}$ \\ School of Mathematical Sciences \\ Fudan University \\ Shanghai 200433, China
}

\begin{abstract}
By constructing an infinite dimensional KAM theorem of the normal frequencies being dense at finitepoint, we show that some shallow water equations such as Benjamin-Bona-Mahony equation and the generalized $d$-Dim. Pochhammer-Chree equation subject to some boundary conditions possess many (a family of initial values of positive Lebesgue measure of finite dimension) smooth solutions which are quasi-periodic in time.
\end{abstract}

\section{Contents}

1 Statement of the main result 2

2 Solution of linear equation for the first Melnikov conditions 8

3 Solution of linear equation for the second Melnikov conditions

4 Iterative Lemma 31

5 Derivation of homological equations

6 Solutions to the homological equations

7 Estimates for new perturbation

8 Proof of the main Theorems

9 Application to Benjamin-Bona-Mahony (BBM) equation 48

10 Application to $d$-dimensional generalized Pochhammer-Chree equation 5

11 Final Remark on global solutions to BBM and gPC

12 Appendices 66

${ }^{*}$ Supported by NNSF of China. (No.11790272 and No.11421061)

†Email:xpyuan@fudan.edu.cn; yuanxiaoping@ hotmail.com 


\section{Statement of the main result}

The general problem discussed here is the persistency of quasi-periodic solutions of linear or integrable partial differential equations (PDEs) after Hamiltonian perturbation, which is closely related to the well-known Kolmogorov-Arnold-Moser (KAM) theory of finite (lower) dimensional invariant tori in smooth infinite dimensional dynamical systems. In this setting the considered PDEs can be written as an infinite dimensional Hamiltonian in the (exterior or interior ) parameter dependent normal form $H_{0}$ subject to a Hamiltonian perturbation $R$

$$
H=H_{0}+R=\left(\omega^{0}(\xi), y\right)+\sum_{j \in \mathbb{Z}^{d}} \lambda_{j}(\xi) z_{j} \bar{z}_{j}+\left\langle B^{0}(\xi) z, \bar{z}\right\rangle+R(x, y, z, \bar{z} ; \xi)
$$

with the symplectic structure

$$
d y \wedge d x+\mathbf{i} \sum_{j \in \mathbb{Z}^{d}} d \bar{z}_{j} \wedge d z_{j}, \mathbf{i}^{2}=-1
$$

where $\xi$ is in some parameter set $\mathscr{O}_{0}$ of positive Lebesgue measure, and $(x, y, z, \bar{z})$ is in the domain $D_{p}\left(s_{0}, r_{0}\right)$ which is to be specified later. The tangent frequencies $\omega^{0}=\left(\omega_{1}^{0}, \cdots, \omega_{N}^{0}\right)$ and the normal frequencies $\lambda_{j}\left(j \in \mathbb{Z}^{d}\right)$ and the linear operator $B^{0}$ depend on $N$ dimensional parameter vector $\xi \in \mathscr{O}_{0} \subset \mathbb{R}^{N}$. Let

$$
\Lambda=\Lambda(\xi)=\operatorname{diag}\left(\lambda_{j}(\xi): j \in \mathbb{Z}^{d}\right) .
$$

Then the Hamiltonian equations of motion of $H_{0}$ with symplectic structure $d y \wedge d x+\mathbf{i} d \bar{z} \wedge d z$ are

$$
\dot{x}=\partial_{y} H_{0}=\omega^{0}(\xi), \dot{y}=-\partial_{x} H_{0}=0, \dot{z}=\mathbf{i}\left(\Lambda(\xi)+B^{0}(\xi)\right) z, \dot{\bar{z}}=-\mathbf{i}\left(\Lambda(\xi)+B^{0}(\xi)\right) \bar{z}
$$

Hence, for each $\xi \in \mathscr{O}_{0}$, there is an invariant $N$-dimensional torus

$$
\mathscr{T}^{N}=\mathbb{T}^{N} \times\{y=0\} \times\{z=0\} \times\{\bar{z}=0\}
$$

with rotational frequencies $\omega^{0}(\xi)$. The main aim of KAM theory is to prove the persistence of the torus $\mathscr{T}_{0}^{N}$, for "most" (in the sense of Lebesgue measure) parameter vector $\xi \in \mathscr{O}_{0}$, under small perturbation $R$ of the Hamiltonian $H_{0}$. Here we give a brief, but not complete at all, history for the infinite dimensional KAM theory to deal with lower dimensional invariant tori. In that direction, Kuksin [29]-[32] and Wayne [44] initiated KAM theory to deal with some partial differential equations of spatial dimension $d=1$ such as 1 dimensional nonlinear Schrödinger equation ([30],[33]) and 1 dimensional nonlinear wave equation ( [42], [44]). Bourgain[11]-[16] developed a new method initiated by Craig-Wayne[19] to deal with the KAM tori for the PDEs in high spatial dimension, based on the Newton iteration, Fröhlich-Spencer techniques, harmonic analysis and semi-algebraic set theory (see [16]). This is called Craig-Wayne-Bourgain (C-W-B) method. We also mention the work by Eliasson-Kuksin[22] and EliassonGrébert-Kuksin[23] where the classical KAM theorem is extended in the direction of [21], [29]-[32], [41] and [44] to deal with higher spatial dimensional nonlinear Schrödinger equation by introducing elegant analysis of TöplitzLipschitz operator. The obtained KAM tori by [22] is linear stable. In addition, the KAM theory is also developed to deal some 1 dimensional PDEs of unbounded perturbation. See, for example, [2, 3, 4, 7, 8, 24, 27, 31, 35, 36 and [46], for the details. In all works mentioned as the above, a basic assumption is that the normal frequencies $\lambda_{j}$ 's cluster to infinity, that is, for some $\kappa>0$,

$$
\lambda_{j} \approx|j|^{\kappa} \rightarrow \infty, \text { as }|j| \rightarrow \infty
$$

We will construct a KAM theorem for

$$
\lambda_{j} \approx \varpi+|j|^{-\kappa} \rightarrow \varpi \neq \infty, \text { as }|j| \rightarrow \infty
$$

where $\varpi$ is a finite real number. 
The present work will contain the first result on the persistency of quasi-periodic solutions and KAM tori for those PDEs with $\lambda_{j}$ 's clustering to a finite limit-point. As for totally new applications of the KAM theorem, we will show that there are many (finite dimensional initial value set of positive Lebesgue measure) KAM tori and quasi-periodic solutions for some kinds of shallow water equations such as Benjamin-Bona-Mahony (BBM) equation (with $\varpi=0$ ):

$$
u_{t}+u_{x}+u u_{x}-u_{x x t}=0, \quad x \in \text { some compact domain in } \mathbb{R}
$$

and the generalized Pochhammer-Chree (gPC) equation (with $\varpi=1$ )

$$
u_{t t}-\Delta u_{t t}-\Delta u+\Delta u^{3}=0, x \in \text { some compact domain in } \mathbb{R}^{d}, d \geq 1 .
$$

In order to state our results, let us introduce some notations. Denote by $d \geq 1$ the spatial dimension of those partial differential equations to be considered. For a $d$-dimensional integer vector $j=\left(j_{1}, \ldots, j_{d}\right) \in \mathbb{Z}^{d}$, define $|j|=\left|j_{1}\right|+\cdots+\left|j_{d}\right|$. For $x \in \mathbb{R}^{N}$ or $x \in \mathbb{C}^{N}$, we also by $|x|$ denote the Euclidean norm of $x$. Given $p>d / 2, \kappa>0$, let $q=p+\kappa$. For $\tilde{p} \in\{p, q\}$, define

$$
h_{\tilde{p}}=\left\{z=\left(z_{j} \in \mathbb{C}: j \in \mathbb{Z}^{d}\right): \quad\|z\|_{\tilde{p}}^{2}=\sum_{j \in \mathbb{Z}^{d}}\left|z_{j}\right|^{2}|j|^{2 \tilde{p}}\right\},
$$

where we take $|j|=1$ as $j=0$ for convenience. This is a Hilbert space with a natural inner product corresponding to the norm $\|\cdot\|_{\tilde{p}}$. Given an integer $N>0$, let $\mathbb{T}_{s}^{N}$ be the complexization of $\mathbb{T}^{N}=\mathbb{R}^{N} /(2 \pi \mathbb{Z})^{N}$ with width $s>0$ :

$$
\mathbb{T}_{s}^{N}=\left\{x \in \mathbb{C}^{N} /\left(2 \pi \mathbb{Z}^{N}\right): \quad|\mathfrak{I} x| \leq s\right\} .
$$

For given $s_{0}>0$ define

$$
\mathscr{P} \tilde{p}=\mathbb{T}_{s_{0}}^{N} \times \mathbb{C}^{N} \times h_{\tilde{p}} \times h_{\tilde{p}}, \quad \tilde{p} \in\{p, q\} .
$$

Take $\mathscr{P}^{p}$ as phase spaces. Denote by $\mathscr{L}\left(h_{\tilde{p}}, h_{\tilde{q}}\right)$ the set consisting of all bounded linear operator from $h_{\tilde{p}} \rightarrow h_{\tilde{q}}$ where $\tilde{p}, \tilde{q} \in\{p, q\}$. Introduce a $D_{p}\left(s_{0}, r_{0}\right)$-neighborhood of the torus $\mathbb{T}^{N} \times\{0\} \times\{0\} \times\{0\}$ in the phase space $\mathscr{P}^{p}$ :

$$
D_{p}\left(s_{0}, r_{0}\right)=\mathbb{T}_{s_{0}}^{N} \times\left\{y \in \mathbb{C}^{N}:|y|<r_{0}^{2}\right\} \times\left\{z \in h_{p}:\|z\|_{p}<r_{0}\right\} \times\left\{\bar{z} \in h_{p}:\|\bar{z}\|_{p}<r_{0}\right\} .
$$

By $X_{\mathscr{G}}$ denote the Hamiltonian vector field for a Hamiltonian function $\mathscr{G}$ defined in $D_{p}\left(s_{0}, r_{0}\right)$ or some sub-domain of $D_{p}\left(s_{0}, r_{0}\right)$ The phase space $\mathscr{P}^{p}$ endowed with $(1.2)$ is a symplectic space. For two vectors $b, c \in \mathbb{C}^{l}$ or $\mathbb{R}^{l}$, we write $(b, c)=\sum_{j=1}^{l} b_{j} c_{j}$ if $\imath<\infty$. If the index $j \in \mathbb{Z}^{d}$, we write $\langle b, c\rangle=\sum_{j \in \mathbb{Z}^{d}} b_{j} c_{j}$.

Assumption A: (Non-degeneracy.) Assume that $\omega^{0}(\xi)=\left(\omega_{1}^{0}(\xi), \ldots, \omega_{N}^{0}(\xi)\right): \mathscr{O}_{0} \subset \mathbb{R}^{N} \rightarrow \mathbb{R}^{N}$ is real continuously differentiable in $\xi \in \mathscr{O}_{0}$ in the sense of Whitney 1 And assume there are two absolute constants $c_{1}, c_{2}>0$ such that

$$
\begin{gathered}
\inf _{\xi \in \mathscr{O}_{0}}\left|\operatorname{det} \partial_{\xi} \omega^{0}(\xi)\right| \geq c_{1}, \\
\sup _{\xi \in \mathscr{O}_{0}}\left|\partial_{\xi} \omega^{0}(\xi)\right| \leq c_{2} .
\end{gathered}
$$

Remark 1. Let $\omega^{0}(\xi)=\omega$. By Assumption $\mathbf{A}$, we can regard $\omega$ as parameter, and $\xi=\left(\omega^{0}\right)^{-1}(\omega): \omega^{0}\left(\mathscr{O}_{0}\right) \rightarrow \mathscr{O}_{0}$. Or assuming $\omega(\xi) \equiv \xi$ without loss of generality.

\footnotetext{
${ }^{1}$ In the following arguments, the differentiability with respect to the parameter $\xi$ is always in the sense of Whitney. We will not mention it again. An alternative way is replacing the Whitney smoothness by Lipschitzian continuity.
} 
Assumption B (The normal frequencies clustering at the origin $0 \in \mathbb{R}$, i.e. $\varpi=0$.) Assume $\lambda_{j}=\lambda_{j}(\xi)$ 's are real and continuously differentiable in $\xi \in \mathscr{O}_{0}$. Assume that there exist constants $c_{11}, c_{12}, c_{13}>0$ and $\kappa>0$ such that

$$
c_{11}|j|^{-\kappa} \leq \lambda_{j}(\xi) \leq c_{12}|j|^{-\kappa}, \quad \forall \xi \in \mathscr{O}_{0}, \quad j \in \mathbb{Z}^{d}
$$

and

$$
\sup _{\xi \in \mathscr{O}_{0}}\left|\partial_{\xi} \lambda_{j}(\xi)\right| \leq c_{13}|j|^{-\kappa}, j \in \mathbb{Z}^{d}
$$

And assume for every $0 \neq k \in \mathbb{Z}^{n}$ and $i, j \in \mathbb{Z}^{d}$,

$$
\frac{d^{*}}{d \omega}\left((k, \omega) \pm \lambda_{i}\right)>0, \frac{d^{*}}{d \omega}\left((k, \omega) \pm\left(\lambda_{i}+\lambda_{j}\right)>0,\right.
$$

where $\lambda_{i}=\lambda_{i}(\xi(\omega))=\lambda_{i}\left(\left(\omega^{0}\right)^{-1}(\omega)\right)$ and $\frac{d^{*}}{d \omega}$ is the directional derivative along the direction such that $\frac{d^{*}}{d \omega}(k, \omega) \geq$ 1.

Remark 2. Note that $\frac{d^{*}}{d \omega}(k, \omega) \geq 1$. By Assumption $\mathbf{A}$ and $(1.8)$, there is $j_{0}>0$ such that, for all $i, j \in \mathbb{Z}^{d}$ with $|i|,|j| \geq j_{0}$, the inequality (1.26) holds true automatically, and there is a constant $c^{*}>0$ such that

$$
\frac{d^{*}}{d \omega}\left((k, \omega) \pm \lambda_{i}\right)>c^{*}, \frac{d^{*}}{d \omega}\left((k, \omega) \pm \lambda_{i} \pm \lambda_{j}\right)>c^{*} .
$$

In addition, if $c_{13}$ is small enough, the inequality $(1.26)$ holds true automatically, too.

Remark 3. By (1.7) and $q=\kappa+p$, we have that for any linear operator $\mathscr{A}: h_{p} \rightarrow h_{q}$

$$
\|\mathscr{A}\|_{h_{p} \rightarrow h_{q}} \leq C|| \Lambda^{-1} \mathscr{A}\left\|_{h_{p} \rightarrow h_{p}},\right\| \Lambda^{-1} \mathscr{A}\left\|_{h_{p} \rightarrow h_{p}} \leq C\right\| \mathscr{A}\left\|_{h_{p} \rightarrow h_{q}},\right\| \mathscr{A} \Lambda^{-1}\left\|_{h_{q} \rightarrow h_{q}} \leq C\right\| \mathscr{A} \|_{h_{p} \rightarrow h_{q}} .
$$

We will consider a Hamiltonian perturbation $R^{0}$ of the integrable Hamiltonian $H_{0}$. In order to define the size of the perturbation $R^{0}$, we need to introduce some norms. Arbitrarily take $s$ and $r$ with $s_{0} \geq s>0, r_{0}>r>0$, $\tilde{p}, \tilde{q} \in\{p, q\}$, and arbitrary take a subset $\mathscr{O} \subset \mathscr{O}_{0}$. For a map $f(x, \xi): \mathbb{T}_{s}^{N} \times \mathscr{O} \rightarrow \mathbb{C}^{N}$, define

$$
|f|_{s, \mathscr{O}}^{2}=\sup _{\xi \in \mathscr{O}} \sum_{k \in \mathbb{Z}^{N}}|\widehat{f}(k, \xi)|^{2} e^{2|k| s},
$$

where $\widehat{f}(k, \xi)$ is the $k$-Fourier coefficient of $f(x, \xi)$ in $x \in \mathbb{T}^{N}$. For a map $f: \mathbb{T}_{s}^{N} \times \mathscr{O} \rightarrow h_{\tilde{p}}$, define

$$
\|f\|_{\tilde{p}, s, \mathscr{O}}^{2}=\sup _{\xi \in \mathscr{O}} \sum_{k \in \mathbb{Z}^{N}}\|\widehat{f}(k, \xi)\|_{\tilde{p}}^{2} e^{2|k| s}, \tilde{p} \in\{p, q\} .
$$

Consider a map $f: D_{\tilde{p}}(s, r) \times \mathscr{O} \rightarrow h_{\tilde{q}}$ with $\tilde{p}, \tilde{q} \in\{p, q\}$. For $(x, y, z, \bar{z}) \in D_{\tilde{p}}(s, r)$, write $f$ into Fourier series in $x$ :

$$
f(x, y, z, \bar{z} ; \xi)=\sum_{k \in \mathbb{Z}^{N}} \widehat{f}(k ; y, z, \bar{z} ; \xi) e^{\mathbf{i}(k, x)} .
$$

Define

$$
\|f(\cdot, y, z, \bar{z} ; \xi)\|_{\tilde{q}, s}^{2}:=\sum_{k \in \mathbb{Z}^{N}} e^{2|k| s}\|\widehat{f}(k ; y, z, \bar{z} ; \xi)\|_{\tilde{q}}^{2} .
$$

The bound $\|f(\cdot, y, z, \bar{z} ; \xi)\|_{\tilde{q}, s}<\infty$ implies that $f(x, y, z, \bar{z} ; \xi)$ as a function of $x$ with its range in $h_{\tilde{q}}$ is analytic in $|\mathfrak{I} x|<s$ and continuous in $|\mathfrak{I} x|=s$. Let

$$
\|f\|_{\tilde{p}, \tilde{q}, s, r, \mathscr{O}}:=\sup _{\xi \in \mathscr{O},|y|<r^{2},\|z\|\left\|_{\tilde{p}}<r,\right\| z \|_{\tilde{p}}<r}\|f(\cdot, y, z, \bar{z} ; \xi)\| \|_{\tilde{q}, s} .
$$


For $f: D_{\tilde{p}}(s, r) \times \mathscr{O} \rightarrow \mathbb{C}^{N}$, define

$$
|f|_{\tilde{p}, s, r, \mathscr{O}}:=\sup _{\xi \in \mathscr{O},|y|<r^{2},\|z\| \mid \tilde{p}<r,\|z\|} \sqrt{\sum_{\tilde{p} \in \mathbb{Z}^{N}}|\widehat{f}(k ; y, z, \bar{z} ; \xi)|^{2} e^{2|k| s}} .
$$

For a map

$$
W=(X, Y, Z, \bar{Z}): D_{\tilde{p}}(s, r) \times \mathscr{O} \subset \mathscr{P} \tilde{p} \times \mathscr{O} \rightarrow \mathscr{P} \tilde{q},
$$

define

$$
|W|_{\tilde{q}, D_{\tilde{p}}(s, r) \times \mathscr{O}}:=\sqrt{|X|_{\tilde{p}, s, r, \mathscr{O}}^{2}+|Y|_{\tilde{p}, s, r, \mathscr{O}}^{2}+\left.|| Z\right|_{\tilde{p}, \tilde{q}, s, r, \mathscr{O}} ^{2}+|| \bar{Z}||_{\tilde{p}, \tilde{q}, s, r, \mathscr{O}}^{2}} .
$$

Following [5] with a minor modification, we introduce the modulus of a function ( vector, matrix or operator). For a scalar complex value function defined on $D_{\tilde{p}}\left(s_{0}, r_{0}\right) \times \mathscr{O}_{0}, \tilde{p} \in\{p, q\}$,

$$
f(x, y, z, \bar{z} ; \xi)=\sum_{k \in \mathbb{Z}^{N}, \gamma \in \mathbb{Z}_{+}^{N} \alpha, \beta \in \mathbb{Z}_{+}^{\mathbb{Z}^{d}}} f_{k, \gamma, \alpha, \beta}(\xi) e^{\mathbf{i}(k, x)} y^{\gamma} z^{\alpha} \bar{z}^{\beta},
$$

we define

$$
\lfloor f\rceil=\lfloor f(x, y, z, \bar{z} ; \xi)\rceil=\sum_{k \in \mathbb{Z}^{N}, \gamma \in \mathbb{Z}_{+}^{N} \alpha, \beta \in \mathbb{Z}_{+}^{\mathbb{Z}^{d}}}\left|f_{k, \gamma, \alpha, \beta}(\xi)\right| e^{\mathbf{i}(k, x)} y^{\gamma} z^{\alpha} \bar{z}^{\beta},
$$

where $\mathbb{Z}_{+}$consists of all non-negative integers. For a vector

$$
f(x, y, z, \bar{z} ; \xi)=\left(f_{j}(x, y, z, \bar{z} ; \xi) \in \mathbb{C}: j \in \mathbb{Z}^{d} \text { or a subset of } \mathbb{Z}^{d}\right),
$$

define

$$
\left.\lfloor f\rceil=\left(\left\lfloor f_{j}(x, y, z, \bar{z} ; \xi)\right\rceil: j \in \mathbb{Z}^{d} \text { or a subset of } \mathbb{Z}^{d}\right)\right) .
$$

For an operator or matrix

$$
f(x, y, z, \bar{z} ; \xi)=\left(f_{i j}(x, y, z, \bar{z} ; \xi) \in \mathbb{C}: i, j \in \mathbb{Z}^{d} \text { or a subset of } \mathbb{Z}^{d}\right),
$$

define

$$
\lfloor f(x, y, z, \bar{z} ; \xi)\rceil=\left(\left\lfloor f_{i j}(x, y, z, \bar{z} ; \xi)\right\rceil: i, j \in \mathbb{Z}^{d} \text { or a subset of } \mathbb{Z}^{d}\right) .
$$

In the whole of this paper, we denote by $C$ a universal constant which may be different in different places and which is independent of the steps of KAM iterations.

Assumption C: (Regularity.) Assume the perturbation term $R^{0}(x, y, z, \bar{z} ; \xi)$ which is defined on the domain $D_{p}\left(s_{0}, r_{0}\right) \times$ $\mathscr{O}_{0}$ is analytic in the space coordinates $(x, y, z, \bar{z})$ and $C^{1}$-smooth in $\xi$ of the parameter vector $\xi \in \mathscr{O}_{0}$, and for each $\xi \in \mathscr{O}_{0}$, the modulus $\left\lfloor X_{R^{0}}\right\rceil$ of its Hamiltonian vector field

$$
X_{R^{0}}:=\left(R_{y}^{0},-R_{x}^{0}, \mathbf{i} \partial_{\bar{z}} R^{0},-\mathbf{i} \partial_{z} R^{0}\right),
$$

defines a analytic map

$$
\left\lfloor X_{R^{0}}\right\rceil: D_{p}\left(s_{0}, r_{0}\right) \subset \mathscr{P}^{p} \rightarrow \mathscr{P}^{q}
$$

satisfying

$$
\left|\left\lfloor X_{R^{0}}\right\rceil\right|_{q, D_{p}\left(s_{0}, r_{0}\right) \times \mathscr{O}_{0}} \leq C \varepsilon_{0},\left|\left\lfloor\partial_{\xi} X_{R^{0}}\right\rceil\right|_{q, D_{p}\left(s_{0}, r_{0}\right) \times \mathscr{O}_{0}} \leq C \varepsilon_{0} .
$$

Assumption D: (Reality.) The Hamiltonian functions $H_{0}(x, y, z, \bar{z} ; \xi)$ and $R^{0}(x, y, z, \bar{z} ; \xi)$ are real when $x$ and $y$ are real and $\bar{z}$ is the complex conjugate of $z$. 
Remark 4. This condition implies that $\omega_{0}(\xi)$ and $\lambda_{j}(\xi)$ 's are real and that $B^{0}(\xi)$ is self-adjoint in the squaresummable space $\ell_{2}$ and

$$
\overline{R^{0}(x, y, z, \bar{z} ; \xi)}=R^{0}(x, y, \bar{z}, z ; \xi) .
$$

Assumption E: For any $\xi \in \mathscr{O}_{0}$, the modulus of the operator $B_{0}$ is relatively small with respect to $q=p+\kappa$ in the following sense:

$$
\sup _{\xi \in \mathscr{O}_{0}}\left\|\left[B^{0}(\xi)\right\rceil\right\|_{h_{p} \rightarrow h_{q}} \leq C \varepsilon_{0}, \sup _{\xi \in \mathscr{O}_{0}}\left\|\left\lfloor\partial_{\xi} B^{0}(\xi)\right\rceil\right\|_{h_{p} \rightarrow h_{q}} \leq C \varepsilon_{0} .
$$

Remark 5. If the modulus $\lfloor B\rceil=\left(\left|B_{i j}\right|: i, j \in \mathbb{Z}^{d}\right)$ of a linear operator $B$ is bounded from $h_{p} \rightarrow h_{q}$, it implies that $B$ is absolutely bounded. See [25] for the notation "absolute boundedness".

Theorem 1.1. $(\varpi=0)$. Suppose that the Hamiltonian $H=H_{0}+R^{0}$ obeys the assumptions $\mathbf{A}, \mathbf{B}, \mathbf{C}, \mathbf{D}, \mathbf{E}$. Then there is a sufficiently small $\varepsilon^{*}=\varepsilon^{*}\left(N, p, q, d, \mathscr{O}_{0}\right)>0$ such that for any $0<\varepsilon_{0}<\varepsilon^{*}$ there is a subset $\mathscr{O} \subset \mathscr{O}_{0}$ and a function $\gamma=\gamma\left(\varepsilon_{0}\right)$ with

$$
\text { Meas } \mathscr{O} \geq\left(\text { Meas } \mathscr{O}_{0}\right)\left(1-O\left(\gamma\left(\varepsilon_{0}\right)\right)\right), \quad \lim _{\varepsilon_{0} \rightarrow 0} \gamma\left(\varepsilon_{0}\right)=0,
$$

and there is a symplectic coordinate change

$$
\Phi: D_{p}\left(\frac{s_{0}}{2}, \frac{r_{0}}{2}\right) \times \mathscr{O} \subset \mathscr{P}^{p} \rightarrow D_{p}\left(s_{0}, r_{0}\right) \times \mathscr{O}_{0} \subset \mathscr{P}^{p},
$$

such that $H=H_{0}+R^{0}$ is changed into

$$
H^{\infty}:=H \circ \Phi=(\omega(\xi), y)+\sum_{j \in \mathbb{Z}^{d}} \lambda_{j}(\xi) z_{j} \bar{z}_{j}+\left\langle B^{\infty}(\xi) z, \bar{z}\right\rangle+R,
$$

where

$$
\begin{gathered}
R=O\left(|y|^{2}+|y|\|z\|_{p}+\|z\|_{p}^{3}\right), \\
\|\left.\left\lfloor X_{R}\right\rceil\right|_{q, D_{p}\left(s_{0} / 2, r_{0} / 2\right) \times \mathscr{O}} \leq C \varepsilon_{0},\left|\left\lfloor\partial_{\xi} X_{R}\right\rceil\right|_{q, D_{p}\left(s_{0} / 2, r_{0} / 2\right) \times \mathscr{O}} \leq C \varepsilon_{0},
\end{gathered}
$$

and for any $\xi \in \mathscr{O}$, the operator $B^{\infty}(\xi)$ obeys

$$
\left\|\left\lfloor B^{\infty}(\xi)-B^{0}(\xi)\right\rceil\right\|_{h_{p} \rightarrow h_{q}} \leq C \varepsilon_{0},\left\|\left\lfloor\partial_{\xi}\left(B^{\infty}(\xi)-B^{0}(\xi)\right)\right\rceil\right\|_{h_{p} \rightarrow h_{q}} \leq C \varepsilon_{0},
$$

and $\omega: \mathscr{O} \rightarrow \mathbb{R}^{N}$ with

$$
\sup _{\xi \in \mathscr{O}}\left|\omega-\omega^{0}\right| \leq C \varepsilon_{0}, \quad \sup _{\xi \in \mathscr{O}}\left|\partial_{\xi}\left(\omega-\omega^{0}\right)\right| \leq C \varepsilon_{0}
$$

Remark 6. The function $\gamma=\gamma\left(\varepsilon_{0}\right)$ can be improved to $\gamma\left(\varepsilon_{0}\right)=\varepsilon_{0}^{1 / 3}$ when the measure of the set $\mathscr{O}_{0}$ is independent of $\varepsilon_{0}$. When $\mathscr{O}_{0}$ depends on $\varepsilon_{0}$, we consider $\mathscr{O}_{0}=\left[0, \sqrt{\varepsilon_{0}}\right]^{N}$, for example. Then $\gamma\left(\varepsilon_{0}\right)=1 /\left|\log \varepsilon_{0}\right|$, and at the same time, (1.20) should be replaced by

$$
\left|\left\lfloor X_{R}\right\rceil\right|_{q, D_{p}\left(s_{0} / 2, r_{0} / 2\right) \times \mathscr{O}} \leq C \varepsilon_{0},\left|\left\lfloor\partial_{\xi} X_{R}\right\rceil\right|_{q, D_{p}\left(s_{0} / 2, r_{0} / 2\right) \times \mathscr{O}} \leq C \sqrt{\varepsilon_{0}},
$$

Thus we always have that $\lim _{\varepsilon_{0} \rightarrow 0}$ Meas $\mathscr{O}=$ Meas $\mathscr{O}_{0}$.

Assumption $\mathbf{B}^{\star}$ Let $\varpi \neq 0$ be a finite real number. Assume $\lambda_{j}=\lambda_{j}(\xi)$ 's are real and continuously differentiable in $\xi \in \mathscr{O}_{0}$. Assume that there exist constants $c_{11}, c_{12}, c_{13}>0$ and $\kappa>0$ such that

$$
c_{11}|j|^{-\kappa} \leq\left|\lambda_{j}(\xi)-\varpi\right| \leq c_{12}|j|^{-\kappa}, \quad \forall \xi \in \mathscr{O}_{0}, \quad j \in \mathbb{Z}^{d}
$$


and

$$
\sup _{\xi \in \mathscr{O}_{0}}\left|\partial_{\xi}\left(\lambda_{j}(\xi)-\varpi\right)\right| \leq c_{13}|j|^{-\kappa}, j \in \mathbb{Z}^{d} .
$$

And assume for every $0 \neq k \in \mathbb{Z}^{n}$ and $i, j \in \mathbb{Z}^{d}$, there is a constant $c^{\star}$ such that

$$
\left|\frac{d^{*}}{d \omega}\left((k, \omega) \pm \lambda_{i}\right)\right|>c^{\star}, \mid \frac{d^{*}}{d \omega}\left((k, \omega) \pm\left(\lambda_{i}+\lambda_{j}\right) \mid>c^{\star},\right.
$$

where $\lambda_{i}=\lambda_{i}(\xi(\omega))=\lambda_{i}\left(\left(\omega^{0}\right)^{-1}(\omega)\right)$ and $\frac{d^{*}}{d \omega}$ is the directional derivative along the direction such that $\frac{d^{*}}{d \omega}(k, \omega) \geq$ 1.

Theorem 1.2. $(\varpi \neq 0)$. Suppose that the Hamiltonian $H=H_{0}+R^{0}$ obeys the assumptions $\mathbf{A}, \mathbf{C}, \mathbf{D}, \mathbf{E}$ and $\mathbf{B}^{\star}$. Then the result of Theorem 1.1 holds true.

Corollary 1. For any $\xi \in \mathscr{O}, \Phi(\mathscr{T})=\Phi\left(\mathbb{T}^{N} \times\{y=0\} \times\{z=0\} \times\{\bar{z}=0\}\right)$ is an invariant torus with rotational frequency $\omega$ for the original Hamiltonian $H=H_{0}+R^{0}$. The torus carries quasi-periodic solutions with frequency $\omega$ for $H$.

Corollary 2. The obtained KAM tori and quasi-periodic solutions are linearly stable.

Remark 7. Since we can by the symplectic transformation $\Phi$ eliminate the quadratic terms (linear part in vector field $\left\langle R^{z z}(x, \xi) z, z\right\rangle+\left\langle R^{\bar{z} \bar{z}}(x, \xi) \bar{z}, \bar{z}\right\rangle$ and reduce $\left\langle R^{z \bar{z}}(x, \xi) z, \bar{z}\right\rangle$ to $\left\langle B^{\infty}(\xi) z, \bar{z}\right\rangle$ where $B^{\infty}(\xi)$ is independent of angle variable $x$, all the obtained KAM tori are linearly stable. However, Not all the persisted tori by KAM technique are linearly stable for PDEs of high spatial dimension $d \geq 2$ when $\lambda_{j} \rightarrow \infty$. Recently, Eliasson-Grebert-Kuksin[23] construct explicit examples of partially hyperbolic KAM tori for $d \geq 2$ dimensional beam equation where the nonlinear perturbation is of the very general form, especially depending on the spatial variable $x \in \mathbb{T}^{d}$. Those hyperbolic KAM tori are situated in the neighborhood of the origin and create around them some local instabilities. It seems possible that the local instabilities lead global instabilities (chaotic motion) by searching for Smale horseshoes via the partially hyperbolic KAM tori. tions

While constructing the (classical) lower dimensional KAM tori, one always needs the first Melnikov condi-

$$
\Delta_{k j}:=\langle k, \omega\rangle+\lambda_{j} \neq 0, \quad \forall k \in \mathbb{Z}^{N}, \forall j \in \mathbb{Z}^{d}
$$

and the second Melnikov conditions

$$
\Delta_{k i j}:=\langle k, \omega\rangle+\lambda_{j}-\lambda_{i} \neq 0, \quad \forall k \in \mathbb{Z}^{N}, \forall i \neq j \in \mathbb{Z}^{d} .
$$

Refer to [37] for the Melnikov conditions. Due to the analyticity of the perturbation, one can assume

$$
|k| \leq K=K_{m} \approx 2^{m}
$$

where $m$ is the step number of the Newton iteration.

- For nonlinear Shrödinger equation, the normal frequencies

$$
\lambda_{j}=\lambda_{j}^{N L S}=|j|^{2}, j \in \mathbb{Z}^{d},
$$

for example. When $|j|>C K$ with $C \gg|\omega|$, we have

$$
\left|\Delta_{k j}\right|>\lambda_{j}^{N L S}-|\langle k, \omega\rangle|>C|j|^{2}-|\omega| K>1,
$$

which is not small.Thus the number of small divisors $\Delta_{k j}$ is finite in the first Melnikov conditions. It is worth to point out that the first Melnikov conditions are unavoidable in both the classical KAM theory for the lower 
dimensional invariant tori and the KAM developed by Craig-Wayne-Bourgain. On the other hand, for BBM and gPC equations, $\lambda_{j}=\lambda_{j}^{\text {shallow }}=\Phi+|j|^{-\kappa}$ with $\kappa>0$. At this time, the normal frequencies $\lambda_{j}^{\text {shallow }}$ cluster to a finite point $\varpi$. We have

$$
\Delta_{k j}=\langle k, \omega\rangle+\lambda_{j}^{\text {shallow }} \rightarrow\langle k, \omega\rangle+\varpi, \text { as }|j| \rightarrow \infty .
$$

Note that zero is a limit point of the set $\left\{\langle k, \omega\rangle+\varpi: k \in \mathbb{Z}^{N}\right\}$. Thus, the number of small divisors $\Delta_{k j}$ is infinite in the first Melnikov conditions for BBM and $\mathrm{gPC}$ equations.

- For NLS, the frequencies $\lambda_{j}^{N L S}$ has the gap property in sense that there is a constant $C$ such that $\mid \lambda_{i}^{N L S}$ $\lambda_{j}^{N L S} \mid>C$ when $\lambda_{i}^{N L S} \neq \lambda_{j}^{N L S}$. Clearly, for BBM and gPC, the gap property does not hold true, since

$$
\lambda_{j}^{\text {shallow }}-\lambda_{i}^{\text {shallow }} \rightarrow 0, \text { as }|i|,|j| \rightarrow \infty .
$$

Incidentally, the gap property does not hold true for nonlinear wave equation of spatial dimension $d \geq 2$. Thus it is an open problem that whether or not there is classical KAM tori which are linearly stable for the nonlinear wave equation with $d \geq 2$.

\section{Solution of linear equation for the first Melnikov conditions}

For a vector (or matrix) value function $f$ defined in $\mathbb{T}_{s}^{N}$ and a large number $K>0$, introduce a cut-off operator $\Gamma=\Gamma_{K}$ as follows:

$$
(\Gamma f)(x)=\left(\Gamma_{K} f\right)(x):=\sum_{|k| \leq K} \widehat{f}(k) e^{\mathbf{i}(k, x)}
$$

where $\widehat{f}(k)$ is the $k$-Fourier coefficient of $f(x)$.

Lemma 2.1. Replacing $\mathscr{O}_{0}, \omega^{0}$ and $B^{0}$ by $\mathscr{O}$, $\omega$ and $B$. Assume $\omega=\omega(\xi), \lambda_{j}=\lambda_{j}(\xi)$ (here $j \in \mathbb{Z}^{d}$ ) defined in $\mathscr{O}$ satisfy Assumptions $\mathbf{A}, \mathbf{B}$, respectively. And assume B satisfies Assumptions $\mathbf{E}$. In addition, assume

$$
R(x, \xi): \mathbb{T}_{s}^{N} \times \mathscr{O} \rightarrow h_{q}
$$

is analytic in $x \in \mathbb{T}_{s}^{N}, C^{1}$ in $\xi \in \mathscr{O}$, and the average

$$
\int_{\mathbb{T}^{N}} R(x, \xi) d x=0, \quad \forall \xi \in \mathscr{O} .
$$

Then there is a subset $\mathscr{O}_{1} \subset \mathscr{O}$ with

$$
\text { Meas } \mathscr{O}_{1}=(\text { Meas } \mathscr{O})\left(1-O\left(K^{-C}\right)\right)
$$

such that for any $\xi \in \mathscr{O}_{1}$, the homological equation

$$
\Gamma\left(\left(\mathbf{i} \omega \cdot \partial_{x}+\Lambda+B(\xi)\right) F(x, \xi)\right)=(\Gamma R)(x, \xi)
$$

has unique solution

$$
F(x, \xi): \mathbb{T}_{s^{\prime}}^{N} \times \mathscr{O}_{1} \rightarrow h_{q}
$$

with $\Gamma F=F$ and

$$
\|\lfloor F\rceil\|_{q, s^{\prime}, \mathscr{O}_{1}} \leq K^{C}\|\lfloor R\rceil\|_{q, s, \mathscr{O}}
$$

and

$$
\left\|\left\lfloor\partial_{\xi} F\right\rceil\right\|_{q, s^{\prime \prime}, \mathscr{O}_{1}} \leq K^{C}\left(\|\left\lfloor[R\rceil\left\|_{q, s, \mathscr{O}}+\right\|\left\lfloor\partial_{\xi} R\right\rceil \|_{q, s, \mathscr{O}}\right),\right.
$$

where $0<s^{\prime \prime}<s^{\prime} \leq s$. 
Proof: By passing (2.5) to Fourier coefficients, we have

$$
(-(k, \omega)+\Lambda+B) \widehat{F}(k)=\widehat{R}(k), \quad \forall k \in \mathbb{Z}^{N}, 0<|k| \leq K,
$$

where $(k, \omega)=(k, \omega) E$ with $E$ being the identity from $h_{q} \rightarrow h_{q}$. In the following, we always by 1 instead of $E$ denote the identity from $h_{\tilde{p}}$ to $h_{\tilde{p}}(\tilde{p} \in\{p, q\})$ or from some finite dimensional space to itself, and write $E x=1 x=x$.

According to Assumption $\mathbf{A}$, assume $\omega(\xi)=\xi$ without loss of generality. In standard procedure, it can be easily proved that there is a subset $\mathscr{O}_{1} \subset \mathscr{O}$ of $\operatorname{Meas}\left(\mathscr{O}_{1}\right) \leq K^{-c_{20}}$ with constant $c_{20}>0$ such that

$$
|(k, \omega(\xi))| \geq K^{-c_{21}}, \quad 0<|k| \leq K, \xi \in \mathscr{O} \backslash \mathscr{O}_{1},
$$

with constant $c_{21}=c_{20}+N$. Recall the Assumption B:

$$
c_{11}|j|^{-\kappa} \leq \lambda_{j} \leq c_{12}|j|^{-\kappa} .
$$

So we choose a constant $c_{22}$ with $c_{22} \gg c_{21}$ such that

$$
\left|\lambda_{j}\right| \leq c_{12} K^{-\kappa c_{22}} \ll K^{-c_{21}}, \quad \text { for }|j| \geq K^{c_{22}} .
$$

Using $K^{c_{22}}$, we partition $\Lambda$ as follows:

$$
\Lambda=\Lambda^{(1)} \oplus \Lambda^{(2)}=\left(\begin{array}{ll}
\Lambda^{(1)} & 0 \\
0 & \Lambda^{(2)}
\end{array}\right)
$$

where $\Lambda^{(1)}=\operatorname{diag}\left(\Lambda_{j}:|j|<K^{c_{22}}\right), \Lambda^{(2)}=\operatorname{diag}\left(\Lambda_{j}:|j| \geq K^{c_{22}}\right)$. In such way, we partition $B$ :

$$
B=\left(\begin{array}{ll}
B^{(11)} & B^{(12)} \\
B^{(21)} & B^{(22)}
\end{array}\right)
$$

where

$$
\begin{array}{ll}
B^{(11)}=\left(B_{i j}:|i|<K^{c_{22}},|j|<K^{c_{22}}\right), & B^{(21)}=\left(B_{i j}:|i| \geq K^{c_{22}},|j|<K^{c_{22}}\right), \\
B^{(12)}=\left(B_{i j}:|i|<K^{c_{22}},|j| \geq K^{c_{22}}\right), & B^{(22)}=\left(B_{i j}:|i| \geq K^{c_{22}},|j| \geq K^{c_{22}}\right)
\end{array}
$$

with $B_{i j}=B_{i j}(\xi)$ being the elements of matrix $B=B(\xi)$. Again in such way, partition the $k$-Fourier coefficients $\widehat{F}(k)$ of $F(x)$ and $\widehat{R}(k)$ of $R(x)$ :

$$
\widehat{F}(k)=\left(\begin{array}{c}
F^{(1)} \\
F^{(2)}
\end{array}\right), \widehat{R}(k)=\left(\begin{array}{c}
R^{(1)} \\
R^{(2)}
\end{array}\right),
$$

where $F^{(1)}=\left(\widehat{F}_{j}(k):|j|<K^{c_{22}}\right)$ and $F^{(2)}=\left(\widehat{F}_{j}(k):|j| \geq K^{c_{22}}\right)$ and so on. And we split $h_{p}=h_{p}^{01} \oplus h_{p}^{02}, h_{q}=$ $h_{q}^{01} \oplus h_{q}^{02}$ and $\ell_{2}=\ell_{2}^{01} \oplus \ell_{2}^{02}$ by advantage of $K^{c_{22}}$. For example,

$$
h_{p}^{01}=\left\{z=\left(z_{j} \in \mathbb{C}\right): j \in \mathbb{Z}^{d},|j|<K^{c_{22}}\right\}
$$

with

$$
\|z\|_{h_{p}^{01}}^{2}=\sum_{j \in \mathbb{Z}^{d},|j|<K^{c 22}}\left|z_{j}\right|^{2}|j|^{2 p}
$$

and

$$
h_{p}^{02}=\left\{z=\left(z_{j} \in \mathbb{C}\right): j \in \mathbb{Z}^{d},|j| \geq K^{c_{22}}\right\}
$$

with

$$
\|z\|_{h_{p}^{02}}^{2}=\sum_{j \in \mathbb{Z}^{d},|j|>K^{c_{22}}}\left|z_{j}\right|^{2}|j|^{2 p} .
$$


Therefore, by the partition as the above, we write

$$
-(k, \omega)+(\Lambda+\widehat{B}(k))=\left(\begin{array}{ll}
-(k, \omega)+\Lambda^{(1)}+B^{(11)} & B^{(12)} \\
B^{(21)} & -(k, \omega)+\Lambda^{(2)}+B^{(22)}
\end{array}\right) .
$$

Let

$$
\begin{aligned}
\mathscr{B}_{1} & :=B^{(11)}-B^{(12)} \mathscr{B}_{2}^{-1} B^{(21)}, \\
\mathscr{B}_{2} & :=-(k, \omega)+\Lambda^{(2)}+B^{(22)}
\end{aligned}
$$

Then by (2.9)

$$
\begin{gathered}
F^{(1)}=\left(-(k, \omega)+\Lambda^{(1)}+\mathscr{B}_{1}\right)^{-1}\left(R^{(1)}-B^{(12)} \mathscr{B}_{2}^{-1} R^{(2)}\right), \\
F^{(2)}=\mathscr{B}_{2}^{-1} R^{(2)}-\mathscr{B}_{2}^{-1} B^{(21)} F^{(1)} .
\end{gathered}
$$

In view of Assumption $\mathbf{E}$ by replacing $B^{0}$ by $B$ and using Remark 3 , one has

$$
\sup _{\xi \in \mathscr{O}}\left\|\Lambda^{-1} B\right\|_{h_{p} \rightarrow h_{p}} \leq \sup _{\xi \in \mathscr{O}}\left\|\Lambda^{-1}\lfloor B\rceil\right\|_{h_{p} \rightarrow h_{p}} \leq C \varepsilon_{0} .
$$

Similarly,

$$
\sup _{\xi \in \mathscr{O}}\left\|\Lambda^{-1} \partial_{\xi} B\right\|_{h_{p} \rightarrow h_{p}} \leq \sup _{\xi \in \mathscr{O}}\left\|\Lambda^{-1}\left\lfloor\partial_{\xi} B\right\rceil\right\|_{h_{p} \rightarrow h_{p}} \leq C \varepsilon_{0} .
$$

It follows by Lemma12.1 in the Appendices, that for $i, j \in\{1,2\}$,

$$
\begin{gathered}
\sup _{\xi \in \mathscr{O}}\left\|\left(\Lambda^{(i)}\right)^{-1} B^{(i j)}\right\| \|_{h_{p}^{0 i} \rightarrow h_{p}^{0 j}} \leq C \varepsilon_{0}, \\
\sup _{\xi \in \mathscr{O}}\left\|\left(\Lambda^{(i)}\right)^{-1} \partial_{\xi} B^{(i j)}\right\|_{h_{p}^{0 i} \rightarrow h_{p}^{0 j}} \leq C \varepsilon_{0} .
\end{gathered}
$$

By (2.12) and (2.20), we have

$$
\begin{aligned}
\left\|\Lambda^{(2)}+B^{(22)}\right\|_{h_{p}^{02} \rightarrow h_{p}^{02}} & =\left\|\Lambda^{(2)}\left(1+\left(\Lambda^{(2)}\right)^{-1} B^{(22)}\right)\right\|_{h_{p}^{02} \rightarrow h_{p}^{02}} \\
& \leq\left\|\Lambda^{(2)}\right\|_{h_{p}^{02} \rightarrow h_{p}^{02}}\left(1+\left\|\left(\Lambda^{(2)}\right)^{-1} B^{(22)}\right\|_{h_{p}^{02} \rightarrow h_{p}^{02}}\right) \\
& \leq C K^{-\kappa c_{22}\left(1+\varepsilon_{0}\right) .}
\end{aligned}
$$

By $\kappa c_{22} \gg c_{21}$ and $|(k, \omega)| \geq K^{-c_{21}}$, it follows that there does exist the inverse of $\mathscr{B}_{2}$ :

$$
\mathscr{B}_{2}^{-1}=\left(-(k, \omega)+\Lambda^{(2)}+B^{(22)}\right)^{-1}=\frac{-1}{(k, \omega)}\left(1-\frac{1}{(k, \omega)}\left(\Lambda^{(2)}+B^{(22)}\right)\right)^{-1}
$$

and

$$
\left\|\mathscr{B}_{2}^{-1}\right\|_{h_{p}^{02} \rightarrow h_{p}^{02}} \leq C \frac{1}{|(k, \omega)|} \leq C K^{c_{21}} .
$$

Moreover, by noting $\left\|\left(\Lambda^{(2)}\right)^{-1} B^{(22)}\right\|_{h_{p}^{02} \rightarrow h_{p}^{02}} \leq C \varepsilon_{0}$ and $\left\|\Lambda^{(2)}\right\|_{h_{p}^{02} \rightarrow h_{p}^{02}} \leq C K^{-\kappa c_{22}} \ll K^{-c_{21}}$,

$$
\begin{aligned}
\left\|\left(\left(\Lambda^{(2)}\right)^{-1} \mathscr{B}_{2}^{-1} \Lambda^{(2)}\right)\right\|_{h_{p}^{02} \rightarrow h_{p}^{02}} & =\left\|\frac{-1}{(k, \omega)}\left(1-\frac{1}{(k, \omega)}\left(1+\left(\Lambda^{(2)}\right)^{-1} B^{(22)}\right) \Lambda^{(2)}\right)^{-1}\right\|_{h_{p}^{02} \rightarrow h_{p}^{02}} \\
& \leq C K^{c_{21}} .
\end{aligned}
$$

By (1.8) in Assumption B,

$$
\left\|\partial_{\xi} \Lambda^{(2)}\right\|_{h_{p}^{02} \rightarrow h_{p}^{02}} \leq C\left\|\Lambda^{(2)}\right\|_{h_{p}^{02} \rightarrow h_{p}^{02}} \leq K^{-\kappa c_{22}} \ll K^{-c_{21}} .
$$


Note

$$
\left|\partial_{\xi}(k, \omega)\right| \leq K
$$

By (2.21), 2.23), 2.24) and 2.26), we have

$$
\left\|\partial_{\xi} \mathscr{B}_{2}^{-1}\right\|_{h_{p}^{02} \rightarrow h_{p}^{02}}=\left\|\mathscr{B}_{2}^{-1}\left(\partial_{\xi} \mathscr{B}_{2}\right) \mathscr{B}_{2}^{-1}\right\|_{h_{p}^{02} \rightarrow h_{p}^{02}} \leq C K^{2 c_{21}+1} .
$$

In addition,

$$
\left\|B^{(12)}\right\|_{h_{p}^{02} \rightarrow h_{p}^{01}} \leq C \varepsilon_{0},\left\|B^{(21)}||_{h_{p}^{01} \rightarrow h_{p}^{02}} \leq\right\| \Lambda^{(2)}\left\|_{h_{p}^{02} \rightarrow h_{p}^{02}}\right\|\left(\Lambda^{(2)}\right)^{-1} B^{(21)} \|_{h_{p}^{01} \rightarrow h_{p}^{02}} \leq C \varepsilon_{0} K^{-\kappa c_{22}}
$$

and

$$
\left\|\partial_{\xi} B^{(12)}\right\|_{h_{p}^{02} \rightarrow h_{p}^{01}} \leq C \varepsilon_{0},\left\|\partial_{\xi} B^{(21)}\right\|_{h_{p}^{01} \rightarrow h_{p}^{02}} \leq\left\|\Lambda^{(2)}\right\|_{h_{p}^{02} \rightarrow h_{p}^{02}}\left\|\left(\Lambda^{(2)}\right)^{-1} \partial_{\xi} B^{(21)}\right\|_{h_{p}^{01} \rightarrow h_{p}^{02}} \leq C \varepsilon_{0} K^{-\kappa c_{22}} .
$$

Applying (2.20), 2.21), 2.23), 2.28) and (2.29) to 2.14), we have

$$
\sup _{\xi \in \mathscr{O}}\left\|\partial_{\xi}^{t} \mathscr{B}_{1}\right\|_{h_{p}^{01} \rightarrow h_{p}^{01}} \leq C \varepsilon_{0}, t=0,1 .
$$

Since $B$ is self-adjoint in $\ell_{2}$, it is easy to see that $\mathscr{B}_{1}$ is Hermitian. Thus, by 12.1 in Appendices,

$$
\left\|\mathscr{B}_{1}\right\|_{\ell_{2}^{01} \rightarrow \ell_{2}^{01}} \leq\left\|\mathscr{B}_{1}\right\|_{h_{p}^{01} \rightarrow h_{p}^{01}} \leq C \varepsilon_{0}
$$

and

$$
\left\|\partial_{\xi} \mathscr{B}_{1}\right\|_{\ell_{2}^{01} \rightarrow \ell_{2}^{11}} \leq\left\|\partial_{\xi} \mathscr{B}_{1}\right\|_{h_{p}^{01} \rightarrow h_{p}^{01}} \leq C \varepsilon_{0}, \quad \xi \in \mathscr{O} \backslash \mathscr{O}_{1}
$$

Choose $\hat{\xi}$ to be a direction such that the directional derivative $\partial_{\hat{\xi}}(k, \omega)=|k|$. (Recall that we have assumed that $\omega(\xi) \equiv \xi)$. Then by 1.10 ,

$$
-\partial_{\hat{\xi}}\left(-(k, \omega)+\Lambda^{(1)}+\mathscr{B}_{1}\right) \geq c^{*}-C \varepsilon_{0} \geq c^{*} / 2,
$$

where $X \geq Y$ means that $X-Y$ is positive for Hermitian matrices $X$ and $Y$. By the variation principle of eigenvalue of matrix (See [28]), any eigenvalue, say $\mu=\mu(\xi)$, of $\left(-\langle k, \omega\rangle+\Lambda^{(1)}+\mathscr{B}_{1}\right)$ satisfies

$$
\left|\partial_{\hat{\xi}} \mu(\xi)\right| \geq c^{*}-C \varepsilon_{0} \geq c^{*} / 2,
$$

where and in the following $C$ is a general constant which might depends on $N, s_{0}, \tau$ and which may be different in different places. Therefore, there is a subset $\mathscr{O}_{2} \subset \mathscr{O} \backslash \mathscr{O}_{1}$ with meas $\mathscr{O}_{2}=O\left(K^{-C_{0} / 2}\right)$ such that for $\forall \xi \in$ $\mathscr{O} \backslash\left(\mathscr{O}_{1} \cup \mathscr{O}_{2}\right)$,

$$
\left\|\left(\left(-(k, \omega)+\Lambda^{(1)}+\mathscr{B}_{1}\right)\right)^{-1}\right\|_{\ell_{2}^{01} \rightarrow \ell_{2}^{01}} \leq K^{C_{0}},
$$

where $C_{0}$ is chosen such that $C_{0}-c_{22}>C_{0} / 2$. By (2.11) and (2.12), we can write $\mathscr{B}_{1}=\left(\mathscr{B}_{1}(i, j):|i|,|j| \leq K^{c_{22}}\right)$, where $\mathscr{B}_{1}(i, j)$ is the matrix elements of $\mathscr{B}_{1}$. Therefore,

$$
\left\|\left(\left(-(k, \omega)+\Lambda^{(1)}+\mathscr{B}_{1}\right)\right)^{-1}\right\|_{h_{p}^{01} \rightarrow h_{p}^{01}} \leq K^{p c_{22}} K^{C_{0}}:=K^{C_{1}} .
$$

Note that $\left\|\left(\Lambda^{(1)}\right)^{-1}\right\|_{h_{p}^{01} \rightarrow h_{p}^{01}} \leq K^{\kappa c_{22}}$. It follows from (2.35), (2.24) and (2.16) that

$$
\left\|\left(\Lambda^{(1)}\right)^{-1} F^{(1)}\right\|_{h_{p}^{01}} \leq K^{C}\left(\left\|\left(\Lambda^{(1)}\right)^{-1} R^{(1)}\right\|_{h_{p}^{01}}+\left\|\left(\Lambda^{(2)}\right)^{-1} R^{(2)}\right\|_{h_{p}^{02}}\right) \leq K^{C}\left\|\Lambda^{-1} \hat{R}(k)\right\|_{h_{p}},
$$


where $C \gg c_{22}$ and $\hat{R}(k)=\hat{R}(k, \xi)$ depends $\xi$. Moreover, by (2.17), 2.25), 2.20) and (2.36),

$$
\begin{aligned}
\left\|\left(\Lambda^{(2)}\right)^{-1} F^{(2)}\right\|_{h_{p}^{02}}= & \left\|\left(\left(\Lambda^{(2)}\right)^{-1} \mathscr{B}_{2}^{-1} \Lambda^{(2)}\right)\left(\Lambda^{(2)}\right)^{-1} R^{(2)}\right\|_{h_{p}^{02}} \\
& +\left\|\left(\left(\Lambda^{(2)}\right)^{-1} \mathscr{B}_{2}^{-1} \Lambda^{(2)}\right)\left(\left(\Lambda^{(2)}\right)^{-1} B^{(21)}\right)\left(\Lambda^{(1)}\right)\left(\left(\Lambda^{(1)}\right)^{-1} F^{(1)}\right)\right\|_{h_{p}^{02}} \\
\leq & C_{1} K^{c_{21}}\left\|\left(\Lambda^{(2)}\right)^{-1} R^{(2)}\right\|_{h_{p}^{2}}+C_{2} K^{c_{21}} \cdot \varepsilon_{0} \cdot\left\|\left(\Lambda^{(1)}\right)^{-1} F^{(1)}\right\|_{h_{p}^{01}} \\
\leq & K^{C}\left\|\Lambda^{-1} \hat{R}(k)\right\|_{h_{p} .}
\end{aligned}
$$

By (2.36) and 2.37),

$$
\|\hat{F}(k)\|_{h_{q}}=\left\|\Lambda^{-1} \hat{F}(k)\right\|_{h_{p}} \leq K^{C}|| \Lambda^{-1} \hat{R}(k) \|_{h_{p}}, \quad \xi \in \mathscr{O}_{m} \backslash\left(\mathscr{O}_{1} \cup \mathscr{O}_{2}\right), \quad 0<|k| \leq K .
$$

Note $\|\widehat{F}(k)\|_{h_{q}}=\|\lfloor\widehat{F}(k)\rceil\|_{h_{q}}$ and $\|\widehat{R}(k)\|_{q}=\|\lfloor\widehat{R}(k)\rceil\|_{q}$. By $(2.38)$,

$$
\begin{aligned}
\| L F\rceil \|_{q, s^{\prime}, \mathscr{O}_{1}}^{2} & =\sum_{0 \neq k \in \mathbb{Z}^{N}}\|\widehat{F}(k)\|_{q}^{2} \cdot e^{2|k| s^{\prime}} \\
& \leq K^{2 C} \sum_{0 \neq k \in \mathbb{Z}^{N}}\|\lfloor\widehat{R}(k)\rceil\|_{q}^{2} \cdot e^{2|k| s} \\
& =\left(K^{C}\|\lfloor R\rceil\|_{q, s, \mathscr{O}}\right)^{2} .
\end{aligned}
$$

It follows that

$$
\|\lfloor F\rceil\|_{q, s^{\prime}, \mathscr{O}_{1}} \leq K^{C}\|\lfloor R\rceil\|_{q, s, \mathscr{O}}
$$

This proves 2.77.

Applying $\partial_{\xi}$ to both sides of (2.5), we have

$$
\Gamma\left(\left(\mathbf{i} \omega \cdot \partial_{x}+\Lambda+B\right) \partial_{\xi} F\right)=\Gamma\left(\partial_{\xi} R\right)-\Gamma\left(\left(\partial_{\xi}\left(\mathbf{i} \omega \cdot \partial_{x}+\Lambda+B\right)\right) F\right) .
$$

Repeating the previous procedure, we can prove 2.8 .

\section{Solution of linear equation for the second Melnikov conditions}

Lemma 3.1. Assume $\mathscr{O}, \omega=\omega(\xi), \lambda_{j}$ 's satisfy Assumptions $\mathbf{A}, \mathbf{B}$, accordingly, by replacing $\mathscr{O}_{0}, \omega^{0}$ by $\mathscr{O}, \omega$. Assume both $B$ and $\breve{B}$ are self-adjoint in the square summable space $\ell_{2}\left(\mathbb{Z}^{d}\right)$ and satisfy Assumption $\mathbf{E}$. And assume the operator-value function $\Lambda^{-1} R$ obeys that

$$
\Lambda^{-1}\lfloor R(x, \xi)\rceil: \mathbb{T}_{s}^{N} \times \mathscr{O} \rightarrow \mathscr{L}\left(h_{p}, h_{p}\right)
$$

is analytic in $x \in \mathbb{T}_{s}^{N}, C^{1}$ in $\xi \in \mathscr{O}$. Then there is a subset $\mathscr{O}_{2} \subset \mathscr{O}$ with

$$
\text { Meas } \mathscr{O}_{2}=(\text { Meas } \mathscr{O})\left(1-O\left(K^{-N}\right)\right)
$$

such that for any $\xi \in \mathscr{O}_{2}$, the homological equation

$$
\Gamma\left(\left(-\mathbf{i} \omega \cdot \partial_{x} \pm(\Lambda+B)\right) F\right) \pm \Gamma(F(\Lambda+\breve{B}))=(\Gamma R)(x, \xi)
$$

has unique solution

$$
F=F(x, \xi)=\widehat{F}(0, \xi)+\sum_{0 \neq k \in \mathbb{Z}^{N},|k| \leq K} \widehat{F}(k, \xi) e^{\mathbf{i}(k, x)}:=\widehat{F}(0, \xi)+\tilde{F}(x, \xi)
$$


fulfilling

$$
\tilde{F}: \mathbb{T}_{s}^{N} \times \mathscr{O}_{2} \rightarrow \mathscr{L}\left(h_{p}, h_{q}\right)
$$

and

$$
\begin{aligned}
& \sup _{x \in \mathbb{T}_{\tilde{s}}^{N}, \xi \in \mathscr{O}_{2}}\|[\tilde{F}\rceil\|_{h_{p} \rightarrow h_{q}} \leq \frac{1}{(s-\tilde{s})^{N}} K^{C} \sup _{x \in \mathbb{T}_{s}^{N}, \xi \in \mathscr{O}}\|\lfloor R\rceil\|_{h_{p} \rightarrow h_{q}}, 0<\tilde{s}<s, \\
& \sup _{x \in \mathbb{T}_{\tilde{s}}^{N}, \xi \in \mathscr{O}_{2}}\left\|\left\lfloor\partial_{\xi} \tilde{F}\right\rceil\right\|_{h_{p} \rightarrow h_{q}} \leq \frac{1}{(s-\tilde{s})^{N}} K^{C} \sup _{x \in \mathbb{T}_{s}^{N}, \xi \in \mathscr{O}}\left(\|\lfloor R\rceil\| h_{h_{p} \rightarrow h_{q}}+\left\|\left\lfloor\partial_{\xi} R\right\rceil\right\|_{h_{p} \rightarrow h_{q}}\right), 0<\tilde{s}<s, \\
& \sup _{\xi \in \mathscr{O}_{2}}\|[\widehat{F}(0, \xi)]\|_{h_{\tilde{p}} \rightarrow h_{\tilde{p}}} \leq K^{C} \sup _{\xi \in \mathscr{O}}\|[\widehat{R}(0, \xi)\rceil\|_{h_{p} \rightarrow h_{q}}, \tilde{p} \in\{p, q\}, \\
& \sup _{\xi \in \mathscr{O}_{2}}\left\|\left\lfloor\partial_{\xi} \widehat{F}(0, \xi)\right\rceil\right\|_{h_{\tilde{p}} \rightarrow h_{\tilde{p}}} \leq K^{C} \sup _{\xi \in \mathscr{O}}\left(\|\lfloor\widehat{R}(0, \xi)\rceil\|_{h_{p} \rightarrow h_{q}}+\left\|\left\lfloor\partial_{\xi} \widehat{R}(0, \xi)\right\rceil\right\|_{h_{p} \rightarrow h_{q}}\right), \tilde{p} \in\{p, q\},
\end{aligned}
$$

where we require that

$$
\int_{\mathbb{T}^{N}} R(x, \xi) d x=0, \quad \forall \xi \in \mathscr{O}
$$

when the sign \pm in (3.3) appears in the form

$$
\pm(\Lambda+B) F \pm F(\Lambda+\breve{B})= \pm((\Lambda+B) F-F(\Lambda+\breve{B})) .
$$

Proof: By passing to Fourier coefficients, we get

$$
((k, \omega) \pm(\Lambda+B)) \widehat{F}(k) \pm \widehat{F}(k)(\Lambda+\breve{B})=\widehat{R}(k), \quad k \in \mathbb{Z}^{N},|k| \leq K .
$$

Case 1. $k \neq 0$.

In order to partition the operator $B$, we need the following lemma.

Lemma 3.2. There are a large constant $c$ and a subset $\mathscr{O}_{4} \subset \mathscr{O}$ with meas $\left(\mathscr{O}_{4}\right) \leq K^{-N}$ such that

$$
\left|(k, \omega) \pm \lambda_{j}\right| \geq \frac{1}{2} K^{-c}, \quad \text { for } k \in \mathbb{Z}^{N}, 0<|k| \leq K, j \in \mathbb{Z}^{d}, \xi \in \mathscr{O} \backslash \mathscr{O}_{4}
$$

Proof. By Assumption A, assume $\omega(\xi) \equiv \xi$ without loss of generality.

Part I: Let $y=(3 d / \kappa)+3>3$. Choose a constant $c>0$ such that

$$
c>\frac{c}{y}>100 N(1+\kappa+d)
$$

and

$$
c\left(1-\frac{3 d}{y \kappa}\right)>100 N(1+\kappa+d) .
$$

By (3.12), there is a subset $\mathscr{O}_{41} \subset \mathscr{O}$ with

$$
\operatorname{meas}\left(\mathscr{O}_{41}\right) \leq K^{-N}
$$

such that for any $\xi \in \mathscr{O} \backslash \mathscr{O}_{41}$,

$$
|(k, \omega)| \geq K^{-\frac{c}{y}}>K^{-c} .
$$

Note $c_{11}|j|^{-\kappa} \leq \lambda_{j} \leq c_{12}|j|^{-\kappa}$. For simplicity, we assume $c_{12}=1$ here. So we have

$$
\begin{aligned}
\left|(k, \omega) \pm \lambda_{j}\right| & \geq K^{-\frac{c}{y}}-\left|\lambda_{j}\right| \\
& \geq K^{-\frac{c}{y}}-|j|^{-\kappa} \\
& \geq(1 / 2) K^{-c}
\end{aligned}
$$


if

$$
|j| \geq\left(2 K^{c / y}\right)^{3 / \kappa}=\left(2^{\frac{1}{\kappa}} K^{\frac{c}{\kappa y}}\right)^{3}:=K_{2} .
$$

In addition, by the definition of $K_{2}$,

$$
\left|\lambda_{j}\right|<\left(\frac{1}{2} K^{-c / y}\right)^{3}, \quad|j|>K_{2} .
$$

Remark 8. If without assuming $c_{12}=1$, one can take $K_{2}=\left(2 c_{12}^{-1} K^{c / y}\right)^{3 / \kappa}$.

Part II: Consider $j$ with $|j|<K_{2}$. Let

$$
\mathscr{O}_{42}:=\bigcup_{\substack{0<|k|<K \\ j j \mid<K_{2}}}\left\{\xi \in \mathscr{O}:\left|(k, \omega) \pm \lambda_{j}\right|<K^{-c}\right\} .
$$

Then by (1.10)

$$
\begin{aligned}
\operatorname{meas} \mathscr{O}_{42} & \leq C K^{-c} K^{N} \cdot\left(2^{\frac{1}{\kappa}} K^{\frac{c}{\kappa y}}\right)^{3 d} \\
& =C 2^{\frac{3 d}{\kappa}} K^{-\left(1-\frac{3 d}{k y}\right) c+N} \\
(\text { by }[3.13) & \leq C K^{-N} .
\end{aligned}
$$

By (3.14) and (3.19), letting $\mathscr{O}_{4}=\mathscr{O}_{41} \cup \mathscr{O}_{42}$, then

$$
\operatorname{meas} \mathscr{O}_{4} \leq C K^{-N}
$$

and using (3.16) and 3.18), one has

$$
\left|(k, \omega) \pm \lambda_{j}\right| \geq \frac{1}{2} K^{-c}, \quad \text { for } \xi \in \mathscr{O} \backslash \mathscr{O}_{4}, j \in \mathbb{Z}^{d},|k| \leq K .
$$

This completes the proof.

Lemma 3.3. Assume that there are real numbers $\mu_{j}=\mu_{j}(\xi)$ 's with $j \in \mathbb{Z}^{d}$ and $|j| \leq K_{2}$ which satisfy

$$
\left|\partial_{\xi} \mu_{j}(\xi)\right| \ll 1 .
$$

Then there is a subset $\mathscr{O}_{4}^{\prime} \subset \mathscr{O}$ with meas $\left(\mathscr{O}_{4}^{\prime}\right) \leq K^{-N}$ such that

$$
\left|(k, \omega) \pm \mu_{j}\right| \geq K^{-c}, \quad \text { for } k \in \mathbb{Z}^{N}, 0<|k| \leq K, j \in \mathbb{Z}^{d},|j| \leq K_{2}, \xi \in \mathscr{O} \backslash \mathscr{O}_{4}^{\prime} .
$$

Proof. The proof is the same as Part II in the proof of Lemma3.2

We are now prepared to partition the matrices $\Lambda$ and $B$. To this end, let

$$
K_{3}=\max \left\{K_{2}^{100(N+1) \kappa y}, 2^{1 / \kappa} K^{100\left(1+\frac{p+\kappa}{\kappa y}\right) \frac{c}{\kappa}}\right\},
$$

where $K_{2}$ is defined in 3.17). By the definition of $K_{3}$, we have

$$
\left|\lambda_{j}\right|<\min \left\{K^{-100 c}, K_{2}^{-10}\right\} \text {, if }|j|>K_{3} .
$$

Recall $\Lambda=\operatorname{diag}\left(\lambda_{j}: j \in \mathbb{Z}^{d}\right)$. Write

$$
\Lambda=\Lambda^{(1)} \oplus \Lambda^{(2)}=\left(\begin{array}{ll}
\Lambda^{(1)} & 0 \\
0 & \Lambda^{(2)}
\end{array}\right)
$$


with $\Lambda^{(1)}=\operatorname{diag}\left(\lambda_{j}:|j| \leq K_{3}\right), \Lambda^{(2)}=\operatorname{diag}\left(\lambda_{j}:|j|>K_{3}\right)$.

According to the partition of $\Lambda$, we partition $B$ as follows

$$
B=\left(\begin{array}{ll}
B^{(11)} & B^{(12)} \\
B^{(21)} & B^{(22)}
\end{array}\right)
$$

where $B^{(11)}=\left(B_{i j}:|i| \leq K_{3},|j| \leq K_{3}\right), B^{(12)}=\left(B_{i j}:|i| \leq K_{3},|j|>K_{3}\right), B^{(21)}=\left(B_{i j}:|i|>K_{3},|j| \leq K_{3}\right)$ and $B^{(22)}=\left(B_{i j}:|i|>K_{3},|j|>K_{3}\right)$. Similarly,

$$
\breve{B}=\left(\begin{array}{cc}
\breve{B}^{(11)} & \breve{B}^{(12)} \\
\breve{B}^{(21)} & \breve{B}^{(22)}
\end{array}\right), \quad \widehat{R}(k)=\left(\begin{array}{ll}
R^{(11)} & R^{(12)} \\
R^{(21)} & R^{(22)}
\end{array}\right), \quad \widehat{F}(k)=\left(\begin{array}{cc}
F^{(11)} & F^{(12)} \\
F^{(21)} & F^{(22)}
\end{array}\right) .
$$

Again according to the partition of $\Lambda$, split $h_{p}=h_{p}^{1} \oplus h_{p}^{2}$, and $\ell_{2}\left(\mathbb{Z}^{d}\right)=\ell_{2}^{1} \oplus \ell_{2}^{2}$, corresponding. It follows, from Assumption $\mathbf{E}$ and Lemma 12.1 in the Appendices, that for $b \in\{B, \breve{B}\}$,

$$
\left\|\left(\Lambda^{(i)}\right)^{-1}\left\lfloor b^{(i j)}\right\rceil\right\|_{h_{p}^{i} \rightarrow h_{p}^{j}} \leq \varepsilon_{0}, \quad\left\|\partial_{\xi}\left(\left(\Lambda^{(i)}\right)^{-1}\left\lfloor b^{(i j)}\right\rceil\right)\right\|_{h_{p}^{i} \rightarrow h_{p}^{j}} \leq \varepsilon_{0}, \quad i, j \in\{1,2\} .
$$

And we have that for $i, j \in\{1,2\}$,

$$
\left\|\left(\Lambda^{(i)}\right)^{-1}\left\lfloor R^{(i j)}\right\rceil\right\|_{h_{p}^{i} \rightarrow h_{p}^{j}} \leq\left\|\Lambda^{-1}\lfloor R\rceil\right\|_{h_{p} \rightarrow h_{p}},\left\|\left(\Lambda^{(i)}\right)^{-1}\left\lfloor\partial_{\xi} R^{(i j)}\right\rceil\right\|_{h_{p}^{i} \rightarrow h_{p}^{j}} \leq\left\|\Lambda^{-1}\left\lfloor\partial_{\xi} R\right\rceil\right\| \|_{h_{p} \rightarrow h_{p}} .
$$

With the above notations, the homological equation 3.10 becomes

$$
\begin{aligned}
& \left(\begin{array}{ll}
(k, \omega) \pm\left(\Lambda^{(1)}+B^{(11)}\right) & B^{(12)} \\
B^{(21)} & (k, \omega) \pm\left(\Lambda^{(2)}+B^{(22)}\right)
\end{array}\right)\left(\begin{array}{ll}
F^{(11)} & F^{(12)} \\
F^{(21)} & F^{(22)}
\end{array}\right) \\
\pm & \left(\begin{array}{ll}
F^{(11)} & F^{(12)} \\
F^{(21)} & F^{(22)}
\end{array}\right)\left(\begin{array}{ll}
\Lambda^{(1)}+\breve{B}^{(11)} & \breve{B}^{(12)} \\
\breve{B}^{(21)} & \Lambda^{(2)}+\breve{B}^{(22)}
\end{array}\right)=\left(\begin{array}{ll}
R^{(11)} & R^{(12)} \\
R^{(21)} & R^{(22)}
\end{array}\right) .
\end{aligned}
$$

For brevity, let

$$
\begin{gathered}
M^{(11)}=(k, \omega) \pm\left(\Lambda^{(1)}+B^{(11)}\right), M^{(22)}=(k, \omega) \pm\left(\Lambda^{(2)}+B^{(22)}\right), M^{(12)}= \pm B^{(12)}, M^{(21)}= \pm B^{(21)} \\
N^{(11)}= \pm\left(\Lambda^{(1)}+\breve{B}^{(11)}\right), N^{(22)}= \pm\left(\Lambda^{(2)}+\breve{B}^{(22)}\right), N^{(12)}= \pm \breve{B}^{(12)}, N^{(21)}= \pm \breve{B}^{(21)} .
\end{gathered}
$$

Then (3.27) becomes

$$
\begin{aligned}
& M^{(11)} F^{(11)}+F^{(11)} N^{(11)}+M^{(12)} F^{(21)}+F^{(12)} N^{(21)}=R^{(11)}, \\
& M^{(22)} F^{(21)}+F^{(21)} N^{(11)}+M^{(21)} F^{(11)}+F^{(22)} N^{(21)}=R^{(21)}, \\
& M^{(11)} F^{(12)}+F^{(12)} N^{(22)}+M^{(12)} F^{(22)}+F^{(11)} N^{(12)}=R^{(12)}, \\
& M^{(22)} F^{(22)}+F^{(22)} N^{(22)}+M^{(21)} F^{(12)}+F^{(21)} N^{(12)}=R^{(22)} .
\end{aligned}
$$

Denote by $X=G_{22}(Y)$ the unique solution of the operator equation

$$
M^{(22)} X+X N^{(22)}=Y .
$$

That is to say, we formally denote by $G_{22}$ the Green function:

$$
G_{22}(\cdot)=\left(M^{(22)}(\cdot)+(\cdot) N^{(22)}\right)^{-1},
$$


where

$$
\left(M^{(22)}(\cdot)+(\cdot) N^{(22)}\right) X=M^{(22)}(X)+(X) N^{(22)} .
$$

Then by (3.33),

$$
F^{(22)}=G_{22}\left(R^{(22)}\right)-G_{22}\left(M^{(21)} F^{(12)}\right)-G_{22}\left(F^{(21)} N^{(12)}\right) .
$$

Similarly, let the Green function $G_{21}$ be formally defined by

$$
G_{21}(\cdot)=\left(M^{(22)}(\cdot)+(\cdot) N^{(11)}-G_{22}\left((\cdot) N^{(12)}\right) N^{(21)}\right)^{-1} .
$$

Then by inserting 3.35) into 3.31,

$$
F^{(21)}=-G_{21}\left(M^{(21)} F^{(11)}\right)+G_{21}\left(G_{22}\left(M^{(21)} F^{(12)}\right) N^{(21)}\right)+\tilde{R}^{(21)},
$$

where

$$
\tilde{R}^{(21)}=G_{21}\left(R^{(21)}-G_{22}\left(R^{(22)}\right) N^{(21)}\right) .
$$

Inserting 3.37) into 3.35, we get

$$
\begin{aligned}
F^{(22)}= & G_{22}\left(R^{(22)}\right)-G_{22}\left(M^{(21)} F^{(12)}\right)+G_{22}\left(G_{21}\left(M^{(21)} F^{(11)}\right) N^{(12)}\right) \\
& -G_{22}\left(G_{21}\left(G_{22}\left(M^{(21)} F^{(12)}\right) N^{(21)}\right) N^{(12)}\right)-G_{22}\left(\tilde{R}^{(21)} N^{(12)}\right) .
\end{aligned}
$$

Inserting 3.39) into 3.32), one has

$$
\begin{aligned}
& M^{(11)} F^{(12)}+F^{(12)} N^{(22)}+M^{(12)} G_{22}\left(G_{21}\left(M^{(21)} F^{(11)}\right) N^{(12)}\right)+F^{(11)} N^{(12)} \\
& -M^{(12)}\left(G_{22}\left(G_{21}\left(G_{22}\left(M^{(21)} F^{(12)}\right) N^{(21)}\right) N^{(12)}\right)+G_{22}\left(M^{(21)} F^{(12)}\right)\right) \\
& =-M^{(12)}\left(G_{22}\left(R^{(22)}\right)-G_{22}\left(\tilde{R}^{(21)} N^{(12)}\right)\right)+R^{(12) .}
\end{aligned}
$$

Let the Green function $G_{12}$ be formally defined by

$$
G_{12}(\cdot)=\left(M^{(11)}(\cdot)+(\cdot) N^{(22)}-M^{(12)}\left(G_{22}\left(G_{21}\left(G_{22}\left(M^{(21)}(\cdot)\right) N^{(21)}\right) N^{(12)}+\left(M^{(21)}(\cdot)\right)\right)\right)\right)^{-1} .
$$

It follows from $(3.40)$ that

$$
F^{(12)}=\mathscr{L}\left(F^{(11)}\right)+\tilde{R}^{(12)}
$$

where

$$
\begin{gathered}
\mathscr{L}\left(F^{(11)}\right):=-G_{12}\left(M^{(12)} G_{22}\left(G_{21}\left(M^{(21)} F^{(11)}\right) N^{(12)}\right)+F^{(11)} N^{(12)}\right), \\
\tilde{R}^{(12)}:=-G_{12}\left(M^{(12)}\left(G_{22}\left(R^{(22)}\right)-G_{22}\left(\tilde{R}^{(21)} N^{(12)}\right)\right)-R^{(12)}\right) .
\end{gathered}
$$

Inserting 3.42 into 3.37, we have

$$
\begin{aligned}
F^{(21)}= & -G_{21}\left(M^{(21)} F^{(11)}\right)+G_{21}\left(G_{22}\left(M^{(21)} \mathscr{L}\left(F^{(11)}\right)\right) N^{(21)}\right) \\
& +G_{21}\left(G_{22}\left(M^{(21)} \tilde{R}^{(12)}\right) N^{(21)}\right)+\tilde{R}^{(21)} .
\end{aligned}
$$

Finally, inserting (3.42) and (3.45) into (3.30), we have

$$
M^{(11)} F^{(11)}+F^{(11)} N^{(11)}+\mathscr{L}_{1}\left(F^{(11)}\right)=\mathscr{R}^{(11)},
$$


where

$$
\begin{aligned}
\mathscr{L}_{1}\left(F^{(11)}\right):=\quad & M^{(12)} G_{21}\left(-M^{(21)} F^{(11)}+G_{22}\left(M^{(21)} \mathscr{L}\left(F^{(11)}\right)\right) N^{(21)}\right) \\
& -G_{12}\left(M^{(12)}\left(G_{22}\left(G_{21}\left(M^{(21)} F^{(11)}\right) N^{(12)}\right)+F^{(11)} N^{(12)}\right)\right) N^{(21)}
\end{aligned}
$$

and

$$
\mathscr{R}^{(11)}:=R^{(11)}-M_{12} G_{21}\left(G_{22}\left(M^{(21)} \tilde{R}^{(12)}\right) N^{(21)}\right)-M^{(12)} \tilde{R}^{(21)}-\tilde{R}^{(12)} N^{(21)} .
$$

Our strategy is as follows:

1. to find $F^{(11)}$ by solving 3.46 ;

2. to find $F^{(12)}$ by solving 3.42 ;

3. to find $F^{(21)}$ by solving 3.37 ;

4. to find $F^{(22)}$ by solving 3.35 .

In order to solve (3.46), we need a more explicit form of $\mathscr{L}_{1}\left(F^{(11)}\right)$. To this end, we introduce the following notations.

Let $\mathscr{H}_{1}$ and $\mathscr{H}_{2}$ be Hilbert spaces. For each $\phi_{1} \in \mathscr{H}_{1}, \phi_{2} \in \mathscr{H}_{2}$, let $\phi_{1} \otimes \phi_{2}$ denote the conjugate bilinear form which acts on $\mathscr{H}_{1} \times \mathscr{H}_{2}$ by

$$
\left(\phi_{1} \otimes \phi_{2}\right)\left\langle\psi_{1}, \psi_{2}\right\rangle=\left(\psi_{1}, \phi_{1}\right)\left(\psi_{2}, \phi_{2}\right), \quad \forall \psi_{1} \in \mathscr{H}_{1}, \psi_{2} \in \mathscr{H}_{2},
$$

where $\left(\psi_{i}, \phi_{i}\right)$ is the inner product of $\phi_{i}$ and $\psi_{i}$ in $\mathscr{H}_{i}(i=1,2)$. Let $\mathscr{E}$ be the set of finite linear combinations of such conjugate linear forms. We define an inner product $(\cdot, \cdot)$ on $\mathscr{E}$ by

$$
(\phi \otimes \psi, \eta \otimes \mu)=(\phi, \eta)(\psi, \mu)
$$

and extending it by linearity to $\mathscr{E}$. By Proposition 1 in pp.49-50 of [43], $(\cdot, \cdot)$ is really an inner product. We define $\mathscr{H}_{1} \otimes \mathscr{H}_{2}$ to be the completion of $\mathscr{E}$ under the inner product $(\cdot, \cdot)$. Let $X$ and $Y$ be densely defined operators on Hilbert spaces $\mathscr{H}_{1}$ and $\mathscr{H}_{2}$ respectively. Let $D(X) \in \mathscr{H}_{1}$ and $D(Y) \in \mathscr{H}_{2}$ be the definition domains of $X$ and $Y$, respectively. Denote by $D(X) \otimes D(Y)$ the set of finite linear combinations of vectors of the form $\phi \otimes \psi$ where $\phi \in D(X)$ and $\psi \in D(Y)$. Then $D(X) \otimes D(Y)$ is dense in $\mathscr{H}_{1} \otimes \mathscr{H}_{2}$. We define $X \otimes Y$ on $D(X) \otimes D(Y)$ by

$$
(X \otimes Y)(\phi \otimes \psi)=X \phi \otimes Y \psi
$$

and extend it by linearity. Then the operator $X \otimes Y$ is well defined. If $X$ and $Y$ are bounded operators on Hilbert space $\mathscr{H}_{1}$ and $\mathscr{H}_{2}$, respectively, then

$$
\|X \otimes Y\|=\|X|\||| Y\|,
$$

where the $\|\cdot\|$ of $\|X \otimes Y\|$ is the operator norm from $\mathscr{H}_{1} \otimes \mathscr{H}_{2}$ to itself and the $\|\cdot\|$ of $\|X\|$ (and $\|Y\|$ ) is the operator norm from $\mathscr{H}_{1}$ (and $\mathscr{H}_{2}$ ) to itself. See Chapter VIII.10 in [43] for the details. By [3.51] one see that if write $X=\left(X_{i j}\right)$ then

$$
X \otimes Y=\left(X_{i j} Y\right),
$$

where $X_{i j}$ 's are the matrix elements of $X$ under the basis of $\mathscr{H}_{1}$. In partitioned multiplication of matrix, we immediately get

$$
(X \otimes Y)(U \otimes V)=(X U) \otimes(Y V)
$$

if the operation above is reasonable. Moreover,

$$
(X \otimes Y)^{-1}=X^{-1} \otimes Y^{-1}
$$


if the inverses exist. In addition, we see from (3.53) that

$$
(X \otimes Y)^{*}=X^{*} \otimes Y^{*}, \quad(\cdot)^{*}=\text { adjoint operator of }(\cdot) .
$$

For a matrix $X=\left(X_{i j} \in \mathbb{C}: i, j \in \mathbb{Z}^{d}\right)$, write $X=\left(\ldots, X_{1}, X_{2}, \ldots, X_{j}, \ldots\right)$ with $X_{j}$ 's are column vectors of $X$. Then formally,

$$
\operatorname{Vec} X=\left(\begin{array}{c}
\vdots \\
X_{1} \\
\vdots \\
X_{j} \\
\vdots
\end{array}\right)
$$

In partitioned multiplication of matrix, then the following holds true formally

$$
\operatorname{Vec}(X U Y)=\left(Y^{T} \otimes X\right) \operatorname{Vec} U
$$

for the three linear operators $X, Y, U$.

With those notations above, by applying "Vec" to 3.46 we get

$$
\left(1 \otimes M^{(11)}+N^{(11) T} \otimes 1+1 \otimes \mathscr{L}_{1}\right) \operatorname{Vec} F^{(11)}=\operatorname{Vec} \mathscr{R}^{(11)} .
$$

By (3.48),

$$
\operatorname{Vec} \mathscr{R}^{(11)}=\operatorname{Vec} R^{(11)}+\operatorname{Vec}\left(-M^{(12)} G_{21}\left(G_{22}\left(M^{(21)} \tilde{R}^{(12)}\right) N^{(21)}\right)-M^{(12)} \tilde{R}^{(21)}-\tilde{R}^{(12)} N^{(21)}\right) .
$$

By applying "Vec" to both left sides and right sides of 3.30)-3.33, we have formally that

$$
\begin{aligned}
& \left(\begin{array}{llll}
1 \otimes M^{(11)}+N^{(11) T} \otimes 1 & 1 \otimes M^{(12)} & N^{(21) T} \otimes 1 & 0 \\
1 \otimes M^{(21)} & 1 \otimes M^{(22)}+N^{(11) T} \otimes 1 & 0 & N^{(21) T} \otimes 1 \\
N^{(12) T} \otimes 1 & 0 & 1 \otimes M^{(11)}+N^{(22) T} \otimes 1 & 1 \otimes M^{(12)} \\
0 & N^{(12) T} \otimes 1 & 1 \otimes M^{(21)} & 1 \otimes M^{(22)}+N^{(22) T} \otimes 1
\end{array}\right) \\
& \left(\begin{array}{l}
\operatorname{Vec} F^{(11)} \\
\operatorname{Vec} F^{(21)} \\
\operatorname{Vec} F^{(12)} \\
\operatorname{Vec} F^{(22)}
\end{array}\right)=\left(\begin{array}{l}
\operatorname{Vec} R^{(11)} \\
\operatorname{Vec} R^{(21)} \\
\operatorname{Vec} R^{(12)} \\
\operatorname{Vec} R^{(22)}
\end{array}\right) .
\end{aligned}
$$

It follows that

$$
\left(1 \otimes M^{(11)}+N^{(11) T} \otimes 1+\mathscr{A}\right) \operatorname{Vec} F^{(11)}=\operatorname{Vec} R^{(11)}+\underline{R},
$$

where

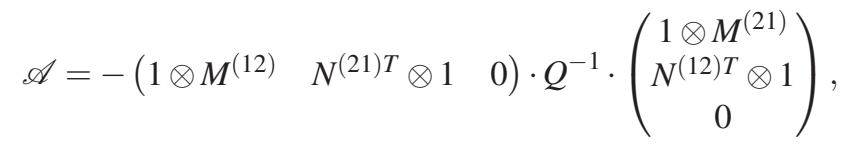

$$
\begin{aligned}
& Q=\left(\begin{array}{lll}
1 \otimes M^{(22)}+N^{(11) T} \otimes 1 & 0 & N^{(21) T} \otimes 1 \\
0 & 1 \otimes M^{(11)}+N^{(22) T} \otimes 1 & 1 \otimes M^{(12)} \\
N^{(12) T} \otimes 1 & 1 \otimes M^{(21)} & 1 \otimes M^{(22)}+N^{(22) T} \otimes 1
\end{array}\right)
\end{aligned}
$$


and

$$
\underline{R}=-\left(\begin{array}{lll}
1 \otimes M^{(12)} & N^{(21) T} \otimes 1 & 0
\end{array}\right) \cdot Q^{-1} \cdot\left(\begin{array}{c}
\operatorname{Vec} R^{(21)} \\
\operatorname{Vec} R^{(12)} \\
\operatorname{Vec} R^{(22)}
\end{array}\right) .
$$

In priori, assume that there exist the inverse of $1 \otimes M^{(11)}+N^{(11) T} \otimes 1+1 \otimes \mathscr{L}_{1}$ and $1 \otimes M^{(11)}+N^{(11) T} \otimes 1+\mathscr{A}$. Regard $R^{(i j)}(i, j \in\{1,2\})$ as "variables" in the left-hand sides of both (3.58) and (3.61). Then comparing (3.58) and (3.61), we get

$$
1 \otimes \mathscr{L}_{1}=\mathscr{A}=\left(\begin{array}{lll}
1 \otimes M^{(12)} & N^{(21) T} \otimes 1 & 0
\end{array}\right) \cdot Q^{-1} \cdot\left(\begin{array}{c}
1 \otimes M^{(21)} \\
N^{(21)} \otimes 1 \\
0
\end{array}\right) .
$$

Using $1 \otimes \mathscr{L}_{1}=\mathscr{A}$ and comparing 3.58 and 3.61 again, we get

$$
\underline{R}=\operatorname{Vec}\left(-M_{12} G_{21}\left(G_{22}\left(M^{(21)} \tilde{R}^{(12)}\right) N^{(21)}\right)-M^{(12)} \tilde{R}^{(21)}-\tilde{R}^{(12)} N^{(21)}\right) .
$$

We are now in position to prove that $\mathscr{A}$ is a self-adjoint operator (i.e., Hermitian matrix) from $\ell_{2}^{1}$ to $\ell_{2}^{1}$. Actually, it is obvious. Recall that $B$ is self-adjoint in $\ell_{2}$. So we have

$$
M^{(i j) *}=M^{(j i)}, \quad N^{(i j) *}=N^{(j i)}, \quad i, j \in\{1,2\},
$$

where the operation $*=$ the complex conjugate plus transpose of matrix. And note that $(X \otimes Y)^{*}=X^{*} \otimes Y^{*}$. It follows that $Q^{*}=Q$. Moreover, $\mathscr{A}^{*}=\mathscr{A}$ if there exists the inverse of $Q$. Now let us prove that there does exist the inverse of $Q$. Let

$$
\mathscr{H}=\left(\begin{array}{c}
h_{p}^{1} \otimes h_{p}^{2} \\
h_{p}^{2} \otimes h_{p}^{1} \\
h_{p}^{2} \otimes h_{p}^{2}
\end{array}\right)=\left(h_{p}^{1} \otimes h_{p}^{2}\right) \oplus\left(h_{p}^{2} \otimes h_{p}^{1}\right) \oplus\left(h_{p}^{2} \otimes h_{p}^{2}\right) .
$$

For $(x, y, z)^{T} \in \mathscr{H}$, define

$$
\left\|(x, y, z)^{T}\right\|_{\mathscr{H}}=\sqrt{\|x\|_{h_{p}^{1} \otimes h_{p}^{2}}^{2}+\|y\|_{h_{p}^{2} \otimes h_{p}^{1}}^{2}+\|z\|_{h_{p}^{2} \otimes h_{p}^{2}}^{2}}
$$

Then $\mathscr{H}$ is a Hilbert space with an inner product corresponding to $\|\cdot\| \mathscr{H}$.

By 3.25, (3.28), 3.29) and using Lemma 12.1 in the Appendices,

$$
\begin{gathered}
\left\|\left\lfloor M^{(i j)}\right\rceil\right\|_{h_{p}^{i} \rightarrow h_{p}^{j}} \leq\left\|\Lambda^{(i)}\right\|_{h_{p}^{i} \rightarrow h_{p}^{i}}\left\|\left(\Lambda^{(i)}\right)^{-1}\left\lfloor M^{(i j)}\right\rceil\right\|_{h_{p}^{i} \rightarrow h_{p}^{j}} \leq \varepsilon_{0}, i \neq j \in\{1,2\}, \\
\left\|\left\lfloor\partial_{\xi} M^{(i j)}\right\rceil\right\|_{h_{p}^{i} \rightarrow h_{p}^{j}} \leq\left\|\Lambda^{(i)}\right\|_{h_{p}^{i} \rightarrow h_{p}^{i}}\left\|\left(\Lambda^{(i)}\right)^{-1}\left\lfloor\partial_{\xi} M^{(i j)}\right\rceil\right\|_{h_{p}^{i} \rightarrow h_{p}^{j}} \leq \varepsilon_{0}, \quad i \neq j \in\{1,2\}, \\
\left\|\left\lfloor N^{(i j)}\right\rceil\right\|_{h_{p}^{i} \rightarrow h_{p}^{j}} \leq \varepsilon_{0},\left\|\left\lfloor\partial_{\xi} N^{(i j)}\right\rceil\right\|_{h_{p}^{i} \rightarrow h_{p}^{j}} \leq \varepsilon_{0}, i \neq j \in\{1,2\}, \\
\left\|\left\lfloor M^{(i i)}-\left((k, \omega) \pm \Lambda^{(i)}\right)\right\rceil\right\|_{h_{p}^{i} \rightarrow h_{p}^{i}} \leq \varepsilon_{0},\left\|\left\lfloor\partial_{\xi}\left(M^{(i i)}-\left((k, \omega) \pm \Lambda^{(i)}\right)\right)\right\rceil\right\|_{h_{p}^{i} \rightarrow h_{p}^{i}} \leq \varepsilon_{0}, \quad i \in\{1,2\}, \\
\left\|\left\lfloor N^{(i i)} \mp \Lambda^{(i)}\right\rceil\right\|_{h_{p}^{i} \rightarrow h_{p}^{i}} \leq \varepsilon_{0},\left\|\left\lfloor\partial_{\xi}\left(N^{(i i)} \mp \Lambda^{(i)}\right)\right\rceil\right\|_{h_{p}^{i} \rightarrow h_{p}^{i}} \leq \varepsilon_{0}, \quad i \in\{1,2\} .
\end{gathered}
$$

By Assumption B,

$$
\|\Lambda\|_{h_{p} \rightarrow h_{p}} \leq C .
$$

By (3.67)-(3.72) and noting that $\|X \otimes Y\|=\|X\|\|Y\|$, we get that each of $Q$ and $\partial_{\xi} Q$ is a bounded linear operator from $\mathscr{H}$ to $\mathscr{H}$. 
Lemma 3.4. (i). The operator

$$
1 \otimes M^{(22)}+N^{(11) T} \otimes 1: h_{p}^{1} \otimes h_{p}^{2} \rightarrow h_{p}^{1} \otimes h_{p}^{2}
$$

has a unique bounded inverse with

$$
\left\|\left\lfloor\left(1 \otimes M^{(22)}+N^{(11) T} \otimes 1\right)^{-1}\right\rceil\right\| \leq K_{2}^{p+\frac{d}{2}} \cdot K^{c} \cdot K^{2 c / y}:=K_{4},
$$

where $\|\cdot\|$ is the operator norm from $h_{p}^{1} \otimes h_{p}^{2}$ to $h_{p}^{1} \otimes h_{p}^{2}$.

(ii). The operator

$$
1 \otimes M^{(11)}+N^{(22) T} \otimes 1: h_{p}^{2} \otimes h_{p}^{1} \rightarrow h_{p}^{2} \otimes h_{p}^{1}
$$

has a unique bounded inverse with

$$
\left\|\left\lfloor\left(1 \otimes M^{(11)}+N^{(22) T} \otimes 1\right)^{-1}\right\rceil\right\| \leq K_{4},
$$

where $\|\cdot\|$ is the operator norm from $h_{p}^{2} \otimes h_{p}^{1}$ to $h_{p}^{2} \otimes h_{p}^{1}$.

(iii). The operator

$$
1 \otimes M^{(22)}+N^{(22) T} \otimes 1: h_{p}^{2} \otimes h_{p}^{2} \rightarrow h_{p}^{2} \otimes h_{p}^{2}
$$

has a unique bounded inverse with

$$
\left\|\left\lfloor\left(1 \otimes M^{(22)}+N^{(22) T} \otimes 1\right)^{-1}\right\rceil\right\| \leq K_{4},
$$

where $\|\cdot\|$ is the operator norm from $h_{p}^{2} \otimes h_{p}^{2}$ to $h_{p}^{2} \otimes h_{p}^{2}$.

Proof. We give the proof only for the case (ii). The remaining proofs are similar.

By (3.28) and 3.29),

$$
1 \otimes M^{(11)}+N^{(22) T} \otimes 1=1 \otimes\left((k, \omega) \pm\left(\Lambda^{(1)}+B^{(11)}\right)\right) \pm\left(\Lambda^{(2)}+\breve{B}^{(22)}\right) \otimes 1 .
$$

Since $B$ is self-adjoint operator from $\ell_{2}$ to $\ell_{2}$ and $\Lambda$ are real diagonal matrix, the matrix $\Lambda^{(1)}+B^{(11)}$ is Hermitian (or self-adjoint in $\ell_{2}^{1}$ ). Make a finer partition of $\Lambda^{(1)}+B^{(11)}$ as follows:

$$
\Lambda^{(1)}=\left(\begin{array}{cc}
\Lambda_{1}^{(1)} & 0 \\
0 & \Lambda_{2}^{(1)}
\end{array}\right)
$$

with $\Lambda_{1}^{(1)}=\operatorname{diag}\left(\lambda_{j}:|j| \leq K_{2}\right)$ and $\Lambda_{2}^{(1)}=\operatorname{diag}\left(\lambda_{j}: K_{2}<|j| \leq K_{3}\right)$. See (3.17) for $K_{2}$ and see (3.23) for $K_{3}$. In this principle as above, make partition

$$
B^{(11)}=\left(\begin{array}{ll}
B_{11}^{(11)} & B_{12}^{(11)} \\
B_{21}^{(11)} & B_{22}^{(11)}
\end{array}\right)
$$

and

$$
h_{p}^{1}=h_{p}^{11} \oplus h_{p}^{12}, \ell_{2}^{1}=\ell_{2}^{11} \oplus \ell_{2}^{12}, h_{q}^{1}=h_{q}^{11} \oplus h_{q}^{12} .
$$

With those partitions, one has a formal equality:

$$
\left(\begin{array}{cc}
1 & -B_{12}^{(11)} \mathscr{B}^{-1} \\
0 & 1
\end{array}\right)\left((k, \omega) \pm\left(\Lambda^{(1)}+B^{(11)}\right)\right)\left(\begin{array}{cc}
1 & 0 \\
-\mathscr{B}^{-1} B_{21}^{(11)} & 1
\end{array}\right)=\left(\begin{array}{cc}
(k, \omega)+\mathscr{C} & 0 \\
0 & \mathscr{B}
\end{array}\right)
$$

where

$$
\mathscr{B}=(k, \omega) \pm\left(\Lambda_{2}^{(1)}+B_{22}^{(11)}\right),
$$




$$
\mathscr{C}= \pm\left(\Lambda_{1}^{(1)}+B_{11}^{(11)}\right)-B_{12}^{(11)}\left((k, \omega) \pm\left(\Lambda_{2}^{(1)}+B_{22}^{(11)}\right)\right)^{-1} B_{21}^{(11)}
$$

By (3.25),

$$
\begin{aligned}
\left\|\left\lfloor B_{22}^{(11)}\right\rceil\right\|_{h_{p}^{12} \rightarrow h_{p}^{12}} & \leq\left\|\Lambda_{2}^{(1)}\right\|_{h_{p}^{12} \rightarrow h_{p}^{12}} \cdot\left\|\left(\Lambda_{2}^{(1)}\right)^{-1}\left\lfloor B_{22}^{(11)}\right\rceil\right\|_{h_{p}^{12} \rightarrow h_{p}^{12}} \\
& \leq \sup \left\{\lambda_{j}:|j| \geq K_{2}\right\}\left\|\Lambda^{-1}\lfloor B\rceil\right\|_{h_{p} \rightarrow h_{p}} \\
& \leq\left((1 / 2) K^{-c / y}\right)^{3} \varepsilon_{0} .
\end{aligned}
$$

Moreover,

$$
\left\|\left\lfloor\Lambda_{2}^{(1)}+B_{22}^{(11)}\right\rceil\right\|_{h_{p}^{12} \rightarrow h_{p}^{12}} \leq \sup _{|j|>K_{2}}\left\{\lambda_{j}\right\}+\left\|\left\lfloor B_{22}^{(11)}\right\rceil\right\|_{h_{p}^{12} \rightarrow h_{p}^{12}} \leq(1 / 8)\left(1+O\left(\varepsilon_{0}\right)\right) K^{-3 c / y} .
$$

Recall that $|(k, \omega)| \geq|K|^{-c / y}$. Using Neumann series and Lemma12.3, we get

$$
\begin{aligned}
\left\|\left\lfloor\mathscr{B}^{-1}\right\rceil\right\|_{h_{p}^{12} \rightarrow h_{p}^{12}} & =\left\|\left\lfloor\left((k, \omega) \pm\left(\Lambda_{2}^{(1)}+B_{22}^{(11)}\right)\right)^{-1}\right\rceil\right\|_{h_{p}^{12} \rightarrow h_{p}^{12}} \\
& =\left\|\frac{1}{|(k, \omega)|}\left\lfloor\left(1 \pm \frac{1}{(k, \omega)}\left(\Lambda_{2}^{(1)}+B_{22}^{(11)}\right)\right)^{-1}\right\rceil\right\|_{h_{p}^{12}} \\
& =\left\|\frac{1}{|(k, \omega)|}\left\lfloor\sum_{j=0}^{\infty}\left( \pm \frac{1}{(k, \omega)}\left(\Lambda_{2}^{(1)}+B_{22}^{(11)}\right)\right)^{j}\right\rceil\right\|_{h_{p}^{12}} \\
& \leq \frac{1}{|(k, \omega)|} \sum_{j=0}^{\infty}\left(\frac{1}{|(k, \omega)|}\left(\left\|\left\lfloor\Lambda_{2}^{(1)}+B_{22}^{(11)}\right\rceil\right\|_{h_{p}^{12}}\right)\right)^{j} \\
& \leq K^{c / y} \sum_{j=0}^{\infty}\left(\frac{1+O\left(\varepsilon_{0}\right)}{2}\right)^{j} \\
& \leq 3 K^{c / y} .
\end{aligned}
$$

Again by 3.25 for $t=0,1$,

$$
\begin{aligned}
\left\|\left\lfloor\partial_{\xi}^{t} B_{21}^{(11)}\right\rceil\right\|_{h_{p}^{11} \rightarrow h_{p}^{12}} & \leq\left\|\Lambda_{2}^{(1)}\right\|_{h_{p}^{12} \rightarrow h_{p}^{12}} \cdot\left\|\left(\Lambda_{2}^{(1)}\right)^{-1}\left\lfloor\partial_{\xi}^{t} B_{21}^{(11)}\right\rceil\right\|_{h_{p}^{11} \rightarrow h_{p}^{12}} \\
& \leq \sup _{|j| \geq K_{2}}\left\{\lambda_{j}\right\}\left\|\Lambda^{-1}\left\lfloor\partial_{\xi}^{t} B\right\rceil\right\| \|_{h_{p} \rightarrow h_{p}} \\
& \leq(1 / 8) \varepsilon_{0} K^{-3 c / y}
\end{aligned}
$$

and, similarly,

$$
\left\|\left\lfloor\partial_{\xi}^{t} B_{12}^{(11)}\right\rceil\right\|_{h_{p}^{12} \rightarrow h_{p}^{11}} \leq \sup _{j \in \mathbb{Z}^{d}}\left\{\lambda_{j}\right\}\left\|\Lambda^{-1}\left\lfloor\partial_{\xi}^{t} B\right\rceil\right\|_{h_{p} \rightarrow h_{p}} \leq C \varepsilon_{0}, t=0,1
$$

Therefore,

$$
\left\|\left\lfloor\partial_{\xi}^{t}\left(B_{12}^{(11)}\left((k, \omega) \pm\left(\Lambda_{2}^{(1)}+B_{22}^{(11)}\right)\right)^{-1} B_{21}^{(11)}\right)\right\rceil\right\|_{h_{p}^{11} \rightarrow h_{p}^{11}} \leq C \varepsilon_{0}^{2} K^{2 c / y} K^{-3 c / y} K \leq \varepsilon_{0} \ll 1 .
$$

By applying (3.25) and 3.84 to 3.79,

$$
\left\|\left\lfloor\partial_{\xi} \mathscr{C}\right\rceil\right\|_{h_{p}^{11} \rightarrow h_{p}^{11}} \leq C \varepsilon_{0} \ll 1 .
$$

Since $B$ is self-adjoint in $\ell_{2}$, it is easy to verify that $\mathscr{C}$ is Hermitian. Thus, by 12.1 ,

$$
\left\|\partial_{\xi} \mathscr{C}\right\|_{\ell_{2} \rightarrow \ell_{2}} \leq\left\|\partial_{\xi} \mathscr{C}\right\|_{h_{p}^{11} \rightarrow h_{p}^{11}} \leq \|\left[\partial_{\xi} \mathscr{C} 7 \|_{h_{p}^{11} \rightarrow h_{p}^{11}} \leq C \varepsilon_{0} \ll 1 .\right.
$$

And since $\mathscr{C}$ is Hermitian, there are a unitary matrix $U=U(\xi)$ and a diagonal matrix $\mathscr{M}=\operatorname{diag}\left(\mu_{j}(\xi): j \in\right.$ $\left.\mathbb{Z}^{d},|j| \leq K_{2}\right)$ such that

$$
\mathscr{C}=U^{*} \mathscr{M} U
$$

where the $\operatorname{star} *$ is the complex conjugate plus transpose. By variation principle of eigenvalues and 3.85 ,

$$
\left|\partial_{\xi} \mu_{j}\right| \leq C \varepsilon_{0} \ll 1
$$


By Lemma 3.3, therefore, there is a subset $\mathscr{O}_{4}^{\prime}$ with its measure $\leq K^{-N}$ such that for $\xi \in \mathscr{O} \backslash \mathscr{O}_{4}^{\prime}$,

$$
\left\|\operatorname{diag}\left((k, \omega)+\mu_{j}:|j| \leq K_{2}\right)^{-1}\right\|_{\ell_{2}^{11} \rightarrow \ell_{2}^{11}} \leq K^{c} .
$$

Note that $\left\|\left.U^{*}\right|_{\ell_{2}^{11} \rightarrow \ell_{2}^{11}}=\right\| U \|_{\ell_{2}^{11} \rightarrow \ell_{2}^{11}}=1$. It follows that

$$
\left\|((k, \omega)+\mathscr{C})^{-1}\right\|_{\ell_{2}^{11} \rightarrow \ell_{2}^{11}} \leq K^{c} .
$$

Let $\mathscr{C}_{i j}$ 's be the elements of $\mathscr{C}$. By the definition of $\mathscr{C}$,

$$
|i| \leq K_{2},|j| \leq K_{2} .
$$

Thus,

$$
\left\|((k, \omega)+\mathscr{C})^{-1}\right\|_{h_{p}^{11} \rightarrow h_{p}^{11}} \leq K_{2}^{p} K^{c} .
$$

Moreover, by Lemma 12.4

$$
\left\|\left\lfloor((k, \omega)+\mathscr{C})^{-1}\right\rceil\right\|_{h_{p}^{11} \rightarrow h_{p}^{11}} \leq K_{2}^{p} K_{2}^{d / 2} K^{c}<K_{4} .
$$

By applying (3.81, 3.82, 3.83) and (3.90) to 3.78), we have

$$
\left\|\left\lfloor\left((k, \omega) \pm\left(\Lambda^{(1)}+B^{(11)}\right)\right)^{-1}\right\rceil\right\|_{h_{p}^{1} \rightarrow h_{p}^{1}} \leq K_{4} .
$$

So

$$
\begin{aligned}
\left.\| L\left(1 \otimes M^{(11)}\right)^{-1}\right\rceil \|_{h_{p}^{2} \otimes h_{p}^{1} \rightarrow h_{p}^{2} \otimes h_{p}^{1}} & =\left\|\left\lfloor 1 \otimes\left(M^{(11)}\right)^{-1}\right\rceil\right\|_{h_{p}^{2} \otimes h_{p}^{1} \rightarrow h_{p}^{2} \otimes h_{p}^{1}} \\
& \left.=\| L\left(M^{(11)}\right)^{-1}\right\rceil \|_{h_{p}^{1} \rightarrow h_{p}^{1}} \\
& \left.=\| L\left((k, \omega) \pm\left(\Lambda^{(1)}+B^{(11)}\right)\right)^{-1}\right\rceil \|_{h_{p}^{1} \rightarrow h_{p}^{1}} \\
& \leq K_{4} .
\end{aligned}
$$

Noting $N^{(22) T}=N^{(22)}$. Again by (3.25) and (3.23),

$$
\begin{aligned}
\left.\|\left\lfloor N^{(22) T}\right) \otimes 1\right\rceil \|_{h_{p}^{2} \otimes h_{p}^{1} \rightarrow h_{p}^{2} \otimes h_{p}^{1}} & =\left\|\left\lfloor\Lambda^{(2)}+\breve{B}^{(22)}\right\rceil\right\|_{h_{p}^{2} \rightarrow h_{p}^{2}} \\
& \leq\left\|\Lambda^{(2)}\right\|_{h_{p}^{2} \rightarrow h_{p}^{2}} \cdot\left\|\left(\Lambda^{(2)}\right)^{-1}\left\lfloor\breve{B}^{(22)}\right\rceil\right\|_{h_{p}^{2} \rightarrow h_{p}^{2}} \\
& \leq \sup _{|j| \geq K_{3}}\left\{\lambda_{j}\right\}\left\|\Lambda^{-1}\lfloor\breve{B}\rceil\right\|_{h_{p} \rightarrow h_{p}} \\
& \leq \varepsilon_{0} K_{3}^{-\breve{K}} .
\end{aligned}
$$

Finally, the proof is finished by using Neumann series, (3.91) and (3.92):

$$
\begin{aligned}
\left.\| L\left(1 \otimes M^{(11)}+N^{(22) T} \otimes 1\right)^{-1}\right\rceil \| & \left.\left.\leq \| L\left(1 \otimes M^{(11)}\right)^{-1}\right\rceil\|\| \| L\left(1+\left(1 \otimes M^{(11)}\right)^{-1}\left(N^{(22) T} \otimes 1\right)\right)^{-1}\right\rceil \| \\
& \left.\left.\leq \| L\left(1 \otimes M^{(11)}\right)^{-1}\right\rceil\left\|\sum_{j=0}^{\infty}\right\| L\left(\left(1 \otimes M^{(11)}\right)^{-1}\left(N^{(22) T} \otimes 1\right)\right)\right\rceil \|^{j} \\
& \leq K_{4} \sum_{j=0}^{\infty}\left(K_{4} K_{3}^{-\kappa} \varepsilon_{0}\right)^{j} \\
& \leq 2 K_{4},
\end{aligned}
$$

where $\|\cdot\|=\|\cdot\|_{h_{p}^{2} \otimes h_{p}^{1} \rightarrow h_{p}^{2} \otimes h_{p}^{1}}$. This completes the proof of the lemma.

By 3.23) ( the definition of $K_{3}$ ) and (3.25),

$$
\begin{aligned}
\left.\| \mid N^{(12) T} \otimes 1\right\rceil \|_{h_{p}^{1} \otimes h_{p}^{2} \rightarrow h_{p}^{2} \otimes h_{p}^{2}} & =\left\|\left\lfloor N^{(12) T}\right\rceil\right\|_{h_{p}^{1} \rightarrow h_{p}^{2}}=\left\|\left\lfloor N^{(21)}\right\rceil\right\|_{h_{p}^{1} \rightarrow h_{p}^{2}} \\
& \leq\left\|\Lambda^{(2)}\right\|_{h_{p}^{2} \rightarrow h_{p}^{2}}\left\|\left(\Lambda^{(2)}\right)^{-1}\left\lfloor N^{(21)}\right\rceil\right\|_{h_{p}^{1} \rightarrow h_{p}^{2}} \\
& \leq \varepsilon_{0} K_{3}^{-\kappa} .
\end{aligned}
$$


Similarly,

$$
\left\|\left\lfloor 1 \otimes M^{(21)}\right\rceil\right\|_{h_{p}^{2} \otimes h_{p}^{1} \rightarrow h_{p}^{2} \otimes h_{p}^{2}} \leq\left\|\left\lfloor M^{(21)}\right\rceil\right\|_{h_{p}^{1} \rightarrow h_{p}^{2}} \leq \varepsilon_{0} K_{3}^{-\kappa} .
$$

By (3.25),

$$
\left\|\left\lfloor N^{(21) T} \otimes 1\right\rceil\right\|_{h_{p}^{2} \otimes h_{p}^{2} \rightarrow h_{p}^{1} \otimes h_{p}^{2}}=\left\|N^{(12)}\right\|_{h_{p}^{2} \rightarrow h_{p}^{1}} \leq \varepsilon_{0}
$$

and

$$
\left\|\left\lfloor 1 \otimes M^{(12)}\right\rceil\right\|_{h_{p}^{2} \otimes h_{p}^{2} \rightarrow h_{p}^{2} \otimes h_{p}^{1}} \leq\left\|\left\lfloor M^{(12)}\right\rceil\right\|_{h_{p}^{2} \rightarrow h_{p}^{1}} \leq \varepsilon_{0} .
$$

In order to see that $Q$ is invertible, we can partition $Q=Q_{1}+Q_{2}$ with

$$
Q_{1}=\left(\begin{array}{lll}
1 \otimes M^{(22)}+N^{(11) T} \otimes 1 & 0 & N^{(21) T} \otimes 1 \\
0 & 1 \otimes M^{(11)}+N^{(22) T} \otimes 1 & 1 \otimes M^{(12)} \\
0 & 0 & 1 \otimes M^{(22)}+N^{(22) T} \otimes 1
\end{array}\right)
$$

and

$$
Q_{2}=\left(\begin{array}{lll}
0 & 0 & 0 \\
0 & 0 & 0 \\
N^{(12) T} \otimes 1 & 1 \otimes M^{(21)} & 0
\end{array}\right)
$$

By (3.93) and (3.94),

$$
\left\|\left\lfloor Q_{2}\right\rceil\right\|_{\mathscr{H} \rightarrow \mathscr{H}} \leq \varepsilon_{0} K_{3}^{-\kappa}
$$

And note that $Q_{1}$ is upper triangle matrix and

$$
\mathscr{Q}_{0} Q_{1}=\left(\begin{array}{ccc}
1 \otimes M^{(22)}+N^{(11) T} \otimes 1 & 0 & 0 \\
0 & 1 \otimes M^{(11)}+N^{(22) T} \otimes 1 & 0 \\
0 & 0 & 1 \otimes M^{(22)}+N^{(22) T} \otimes 1
\end{array}\right),
$$

where

$$
\mathscr{Q}_{0}=\left(\begin{array}{ccc}
1 & 0 & -\left(1 \otimes M^{(22)}+N^{(22) T} \otimes 1\right)^{-1}\left(1 \otimes N^{(21) T}\right) \\
0 & 1 & -\left(1 \otimes M^{(22)}+N^{(22) T} \otimes 1\right)^{-1}\left(1 \otimes M^{(12)}\right) \\
0 & 0 & 1
\end{array}\right)
$$

Also note

$$
\mathscr{Q}_{0}^{-1}=\left(\begin{array}{ccc}
1 & 0 & \left(1 \otimes M^{(22)}+N^{(22) T} \otimes 1\right)^{-1}\left(1 \otimes N^{(21) T}\right) \\
0 & 1 & \left(1 \otimes M^{(22)}+N^{(22) T} \otimes 1\right)^{-1}\left(1 \otimes M^{(12)}\right) \\
0 & 0 & 1
\end{array}\right)
$$

In view of (3.95), 3.96) and Lemma 3.4,

$$
\left\|\left\lfloor Q_{1}^{-1}\right\rceil\right\|_{\mathscr{H} \rightarrow \mathscr{H}} \leq K_{4}^{2} .
$$

Note that $K_{4}^{2} \ll K_{3}^{K}$. In view of (3.93), (3.94) and using Neumann series, it is easy to get

$$
\left\|\left\lfloor Q^{-1}\right\rceil\right\|_{\mathscr{H} \rightarrow \mathscr{H}}=\left\|\left\lfloor Q_{1}^{-1}\left(1+Q_{1}^{-1} Q_{2}\right)^{-1}\right\rceil\right\|_{\mathscr{H} \rightarrow \mathscr{H}} \leq C K_{4}^{2} .
$$

By (3.25),

$$
\left\|\left\lfloor\partial_{\xi} Q\right\rceil\right\|_{\mathscr{H} \rightarrow \mathscr{H} \leq C K}
$$


In view of (3.25), (3.64), (3.97) and (3.98),

$$
\begin{aligned}
& \left\|\left\lfloor\partial_{\xi}\left(1 \otimes \mathscr{L}_{1}\right)\right\rceil\right\|_{h_{p}^{1} \otimes h_{p}^{1}}=\mid\left\|\left\lfloor\partial_{\xi} \mathscr{A}\right\rceil\right\|_{h_{p}^{1} \otimes h_{p}^{1}} \\
\leq & \left\|\left\lfloor\partial_{\xi} Q^{-1}\right\rceil\right\|_{\mathscr{H} \rightarrow \mathscr{H}}\left(\left\|\left(\Lambda^{2}\right)^{-1}\left\lfloor M^{(21)}\right\rceil\right\|_{h_{p}^{1} \rightarrow h_{p}^{2}}+\left\|\left(\Lambda^{2}\right)^{-1}\left\lfloor N^{(21) T}\right\rceil\right\|_{h_{p}^{1} \rightarrow h_{p}^{2}}\right) \sup _{|j|>K_{3}}\left|\lambda_{j}\right| \\
\leq & \varepsilon_{0} C K K_{4}^{4} K_{3}^{-\kappa} \ll K^{-c} .
\end{aligned}
$$

By (12.1) in Appendices,

$$
\left\|\partial_{\xi}\left(1 \otimes \mathscr{L}_{1}\right)\right\|_{\ell_{2}^{1} \rightarrow \ell_{2}^{1}}=\left\|\partial_{\xi} \mathscr{A}\right\|_{\ell_{2}^{1} \rightarrow \ell_{2}^{1}} \leq\left\|\left\lfloor\partial_{\xi} \mathscr{A}\right\rceil\right\|_{h_{p}^{1} \rightarrow h_{p}^{1}} \leq K^{-c} \ll \varepsilon_{0} .
$$

Note

$$
1 \otimes M^{(11)}+N^{(11) T} \otimes 1+\mathscr{A}=1 \otimes((k, \omega)) \pm 1 \otimes B^{(11)} \pm \breve{B}^{(11) T} \otimes 1+\mathscr{A} .
$$

By (1.10), (3.25) and (3.100), we have

$$
\partial_{k(\xi)}\left(1 \otimes M^{(11)}+N^{(11) T} \otimes 1+\mathscr{A}\right) \geq c^{*}-C \varepsilon_{0}>c^{*} / 2>0,
$$

where $k(\xi)$ is the direction derivative such that $\left(k(\xi) \cdot \partial_{\xi}\right)((k, \xi))=|k|$. (We have used the notation $X \geq Y$ when $X-Y$ is a Hermitian positive definite matrix for Hermitian matrices $X$ and $Y$. ) In addition, $1 \otimes M^{(11)}+N^{(11) T} \otimes$ $1+\mathscr{A}$ is obviously Hermitian, since $B$ is self-adjoint in $\ell_{2}$. Let $\mu=\mu(\xi)$ be any eigenvalue of $1 \otimes M^{(11)}+N^{(11) T} \otimes$ $1+\mathscr{A}$. Then

$$
\left|\partial_{k(\xi)} \mu(\xi)\right| \geq c^{*} / 2
$$

by the variation of eigenvalues for Hermitian matrix. Moreover, there is a subset $\mathscr{O}_{2} \subset \mathscr{O}$ with Meas $\mathscr{O}_{2} \leq K^{-N}$ such that for any $\xi \in \mathscr{O} \backslash \mathscr{O}_{2}$,

$$
\left\|\left(1 \otimes M^{(11)}+N^{(11) T} \otimes 1+\mathscr{A}\right)^{-1}\right\|_{\ell_{2}^{1} \otimes \ell_{2}^{1} \rightarrow \ell_{2}^{1} \otimes \ell_{2}^{1}} \leq K^{C},
$$

where $C$ is chosen large enough such that $K^{-C} \cdot K_{3}^{d}<K^{-N}$. Thus, by (3.58),

$$
\left\|\operatorname{Vec} F^{(11)}\right\|_{\ell_{2}^{1} \otimes \ell_{2}^{1}} \leq K^{C}\left\|\mathscr{R}^{(11)}\right\|_{\ell_{2}^{1} \otimes \ell_{2}^{1}} \text {. }
$$

Now we are in position to estimate $\left\|\mathscr{R}^{(11)}\right\|_{\ell_{2}}$. To that end, we introduce some notations. For $\tilde{p}, \tilde{q} \in\{p, q\}$, let $h_{\tilde{p}, \tilde{q}}^{i j}=\mathscr{L}\left(h_{\tilde{p}}^{i} \rightarrow h_{\tilde{q}}^{j}\right)$ be the set of all the bounded linear operators from $h_{\tilde{p}}^{i}$ to $h_{\tilde{q}}^{j}$, (recalling $\left.h_{\tilde{p}}=h_{\tilde{p}}^{1} \oplus h_{\tilde{p}}^{2}\right)$, and for any $X \in h_{\tilde{p}, \tilde{q}}^{i j}$, define

$$
\|X\|_{h_{\tilde{p}, \tilde{q}}^{i j}}=\|X\|_{h_{\tilde{p}}^{i} \rightarrow h_{\tilde{q}}^{j}}
$$

Then $\left(h_{\tilde{p}, \tilde{q}}^{i j},\|\cdot\|_{h_{\tilde{p}, \tilde{q}}^{i j}}\right)$ is a Banach space for $i, j \in\{1,2\}, \tilde{p}, \tilde{q} \in\{p, q\}$.

Lemma 3.5. (i). Arbitrarily take $\lfloor Y\rceil \in h_{p, q}^{22}$ (it follows $\left(\Lambda^{(2)}\right)^{-1}\lfloor Y\rceil \in h_{p, p}^{22}$ and $\lfloor Y\rceil\left(\Lambda^{(2)}\right)^{-1} \in h_{q, q}^{22}$ ). Then $\left\lfloor G_{22}(Y)\right\rceil \in h_{p, p}^{22} \cap h_{q, q}^{22}$ and

$$
\left\|\left(\Lambda^{(2)}\right)^{-1}\left\lfloor G_{22}(Y)\right\rceil\right\|_{h_{p, p}^{22}} \leq 2 K^{c}\left\|\left(\Lambda^{(2)}\right)^{-1}\lfloor Y\rceil\right\|_{h_{p, p}^{22}},\left\|\left\lfloor G_{22}(Y)\right\rceil\left(\Lambda^{(2)}\right)^{-1}\right\|_{h_{q, q}^{22}} \leq 2 K^{c}||\lfloor Y\rceil\left(\Lambda^{(2)}\right)^{-1} \|_{h_{q, q}^{22}}
$$

(ii). Arbitrarily take $\lfloor Y\rceil \in h_{p, q}^{12}$ (it follows $\left(\Lambda^{(2)}\right)^{-1}\lfloor Y\rceil \in h_{p, p}^{12}$ and $\lfloor Y\rceil\left(\Lambda^{(1)}\right)^{-1} \in h_{q, q}^{12}$ ). Then $\left\lfloor G_{21}(Y)\right\rceil \in$ $h_{p, p}^{12} \cap h_{q, q}^{12}$ and

$$
\left\|\left(\Lambda^{(2)}\right)^{-1}\left\lfloor G_{21}(Y)\right\rceil\right\|_{h_{p, p}^{12}} \leq K_{5}||\lfloor Y\rceil\left\|_{h_{p, q}^{12}},\right\|\left\lfloor G_{21}(Y)\right\rceil\left(\Lambda^{(1)}\right)^{-1}\left\|_{h_{q, q}^{12}} \leq K_{5}\right\|\lfloor Y\rceil \|_{h_{p, q}^{12}}, K_{5}:=K^{4 c q} .
$$


(iii). Arbitrarily take $\lfloor Y\rceil \in h_{p, q}^{21}$ (it follows $\left(\Lambda^{(1)}\right)^{-1}\lfloor Y\rceil \in h_{p, p}^{21}$ and $\lfloor Y\rceil\left(\Lambda^{(2)}\right)^{-1} \in h_{q, q}^{21}$ ). Then $\left\lfloor G_{12}(Y)\right\rceil \in h_{p, p}^{21} \cap$ $h_{q, q}^{21}$ and

$$
\left\|\left\lfloor G_{12}(Y)\right\rceil\left(\Lambda^{(2)}\right)^{-1}\right\|_{h_{p, p}^{21}} \leq K_{5}\|\lfloor Y\rceil\|_{h_{p, q}^{21}},\left\|\left(\Lambda^{(1)}\right)^{-1}\left\lfloor G_{12}(Y)\right\rceil\right\|_{h_{q, q}^{21}} \leq K_{5}\|\lfloor Y\rceil\|_{h_{p, q}^{21}}
$$

Proof. Recall $\left|\lambda_{j}\right| \leq K_{3}^{-1 / \kappa}$ for $|j|>K_{3}$. By Assumption $\mathbf{E}$,

$$
\begin{aligned}
& \left\|\left\lfloor b^{(22)}\right\rceil\right\|_{h_{p, p}^{22}} \leq \sup _{|j|>K_{3}}\left|\lambda_{j}\right|\left\|\left(\Lambda^{(2)}\right)^{-1}\lfloor b\rceil\right\|_{h_{p, p}^{22}} \leq \varepsilon_{0} K_{3}^{-\kappa}, b \in\{B, \breve{B}\}, \\
& \left\|\left[b^{(22)}\right\rceil\right\|_{h_{q, q}^{22}} \leq \sup _{|j|>K_{3}}\left|\lambda_{j}\right|||\lfloor b\rceil\left(\Lambda^{(2)}\right)^{-1} \|_{h_{q, q}^{22}} \leq \varepsilon_{0} K_{3}^{-\kappa}, b \in\{B, \breve{B}\}, \\
& \left\|\left\lfloor\Lambda^{(2)}+b^{(22)}\right\rceil\right\|_{h_{p, p}^{22}} \leq C K_{3}^{-\kappa},\left\|\left\lfloor\Lambda^{(2)}+b^{(22)}\right\rceil\right\|_{h_{q, q}^{22}} \leq C K_{3}^{-\kappa}, b \in\{B, \breve{B}\} .
\end{aligned}
$$

Proof of $(i)$. Consider the operator equation with the unknown variable $X$ :

$$
M^{(22)} X+X N^{(22)}=Y \text {. }
$$

Recall (3.28),

$$
M^{(22)}=(k, \omega) \pm\left(\Lambda^{(2)}+B^{(22)}\right), \quad N^{(22)}= \pm\left(\Lambda^{(22)}+\breve{B}^{(22)}\right) .
$$

Let

$$
\mathscr{N}_{l}= \pm\left(\Lambda^{(2)}+B^{(22)}\right), \mathscr{N}_{r}= \pm\left(\Lambda^{(22)}+\breve{B}^{(22)}\right) .
$$

Note that the $(k, \omega)$ in the linear operator $M^{(22)}$ is a product by a scalar $(k, \omega)$ and an identity map. So the equation (3.105) can be rewritten as

$$
\left(\frac{(k, \omega)}{2}+\mathscr{N}_{l}\right) X+X\left(\frac{(k, \omega)}{2}+\mathscr{N}_{r}\right)=Y
$$

Without loss of generality, we can assume $(k, \omega)>0$. Thus, by (3.15), we have $(k, \omega)>K^{-c / y}>0$. According to (3.104,

$$
\left\|\mathscr{N}_{l}\right\|_{h_{\tilde{p}, \tilde{p}} 2}^{22}<C K_{3}^{-\kappa},\left\|\mathscr{N}_{r}\right\|_{h_{\tilde{p}, \tilde{p}}}^{22}<C K_{3}^{-\kappa}, \tilde{p} \in\{p, q\} .
$$

Note $K_{3}^{-\kappa} \ll K^{-c / y}$. Thus for any $t>0$,

$$
\left\|\left\lfloor\exp \left(-t\left(\frac{(k, \omega)}{2}+\mathscr{N}_{l}\right)\right)\right\rceil\right\|_{h_{\tilde{p}, \tilde{p}}} \leq e^{-\frac{t}{2}\left(K^{-c / y}-K_{3}^{-\kappa}\right)} \leq e^{-(t / 4) K^{-c / y}}
$$

and

$$
\left\|\left\lfloor\exp \left(-t\left(\frac{(k, \omega)}{2}+\mathscr{N}_{r}\right)\right)\right\rceil\right\|_{h_{\tilde{p}, \tilde{p}}} \leq e^{-\frac{t}{2}\left(K^{-c / y}-K_{3}^{-\kappa}\right)} \leq e^{-(t / 4) K^{-c / y}}
$$

Thus

$$
X=\int_{0}^{\infty} \exp \left(-t\left(\frac{(k, \omega)}{2}+\mathscr{N}_{l}\right)\right) Y \exp \left(-t\left(\frac{(k, \omega)}{2}+\mathscr{N}_{r}\right)\right) d t
$$

is well-defines and solves (3.105). Moreover,

$$
\left(\Lambda^{(2)}\right)^{-1} X=\int_{0}^{\infty} \exp \left(-t\left(\frac{(k, \omega)}{2}+\left(\Lambda^{2)}\right)^{-1} \mathscr{N}_{l} \Lambda^{(2)}\right)\right)\left(\left(\Lambda^{(2)}\right)^{-1} Y\right) \exp \left(-t\left(\frac{(k, \omega)}{2}+\mathscr{N}_{r}\right)\right) d t .
$$

Thus,

$$
\left\|\left(\Lambda^{(2)}\right)^{-1}\lfloor X\rceil\right\|_{h_{p, p}} \leq\left\|\left(\Lambda^{2)}\right)^{-1}\lfloor Y\rceil\right\|_{h_{p, p}} \int_{0}^{\infty} e^{-\frac{t}{2} K^{-c / y}} d t=2 K^{c / y}\|\lfloor Y\rceil\|_{h_{p, q}} \leq 2 K^{c}\|\lfloor Y\rceil\|_{h_{p, q}} .
$$

Similarly,

$$
\left\|\lfloor X\rceil\left(\Lambda^{2)}\right)^{-1}\right\|_{h_{p, p}} \leq 2 K^{c}\|\lfloor Y\rceil\|_{h_{p, q}} .
$$


This completes the proof of (i).

Proof of (ii). By the definition of $G_{21}$ (See (3.36) $)$, the operator valued equation $G_{21}(Y)=X$ reads

$$
\pm\left(\Lambda^{(2)}+B^{(22)}\right) X+X\left((k, \omega) \pm\left(\Lambda^{(11)}+B^{(11)}\right)\right)-G_{22}\left(X\left( \pm \breve{B}^{(12)}\right)\right)\left( \pm \breve{B}^{(21)}\right)=Y,
$$

where we used $(k, \omega) X=X(k, \omega)$. Set $\mathscr{I}:=(k, \omega) \pm\left(\Lambda^{(11)} \pm \breve{B}^{(11)}\right)$. By (3.78) and (3.87), one has

$$
\mathscr{I}^{-1}=\left(\begin{array}{cc}
1 & \breve{B}_{12}^{(11)} \mathscr{B}^{-1} \\
0 & 1
\end{array}\right)\left(\begin{array}{cc}
U & 0 \\
0 & 1
\end{array}\right)\left(\begin{array}{cc}
(k, \omega)+\mathscr{M})^{-1} & 0 \\
0 & \mathscr{B}^{-1}
\end{array}\right)\left(\begin{array}{cc}
U^{*} & 0 \\
0 & 1
\end{array}\right)\left(\begin{array}{cc}
1 & 0 \\
\mathscr{B}^{-1} \breve{B}_{21}^{(11)} & 1
\end{array}\right),
$$

where $\mathscr{B}=(k, \omega) \pm\left(\Lambda_{2}^{(1)}+\breve{B}_{22}^{(11)}\right)$ and the star $*$ means complex conjugate plus transpose.

In view of the partition (3.76) and (3.77), and using Lemma 12.1 in the Appendices, and Assumption $\mathbf{E}$, we have that for $\tilde{p} \in\{p, q\}$ and $b \in\{B, \breve{B}\}$,

$$
\begin{aligned}
\left.\| \mid b_{22}^{(11)}\right\rceil \|_{h_{\tilde{p}}^{12} \rightarrow h_{\tilde{p}}^{12}} & \left.\left.\left.\| \mid b_{12}^{(11)}\right\rceil\left\|_{h_{\tilde{p}}^{12} \rightarrow h_{\tilde{p}}^{11}},\right\| \mid b_{21}^{(11)}\right\rceil\left\|_{h_{\tilde{p}}^{11} \rightarrow h_{\tilde{p}}^{12}} \leq\right\||| b^{(11)}\right\rceil \|_{h_{\tilde{p}}^{11} \rightarrow h_{\tilde{p}}^{11}} \leq \varepsilon_{0}, \\
\left\|\left\lfloor\Lambda_{2}^{(1)}+b_{22}^{(11)}\right\rceil\right\|_{h_{p}^{12} \rightarrow h_{p}^{12}} & \leq\left\|\Lambda_{2}^{(1)}\right\|\left\|_{h_{p}^{12} \rightarrow h_{p}^{12}}+\right\| \Lambda_{2}^{(1)}\|\|_{h_{p}^{12} \rightarrow h_{p}^{12}}\left\|\left(\Lambda_{2}^{(1)}\right)^{-1}\left\lfloor b_{22}^{(11)}\right\rceil\right\|_{h_{p}^{12} \rightarrow h_{p}^{12}} \\
& \leq\left(1+\varepsilon_{0}\right) \sup _{|j|>K_{2}}\left|\lambda_{j}\right| \leq\left(1+\varepsilon_{0}\right) K_{2}^{-K} \\
& \leq \frac{1}{2}\left(1+\varepsilon_{0}\right) K^{-c / y}
\end{aligned}
$$

and

$$
\begin{aligned}
\left\|\left\lfloor\Lambda_{2}^{(1)}+b_{22}^{(11)}\right\rceil\right\|_{h_{q}^{12} \rightarrow h_{q}^{12}} & \leq\left\|\Lambda_{2}^{(1)}\right\|_{h_{q}^{12} \rightarrow h_{q}^{12}}+\left\|\Lambda_{2}^{(1)}\right\|_{h_{q}^{12} \rightarrow h_{q}^{12}}\left\|\left\lfloor b_{22}^{(11)}\right\rceil\left(\Lambda_{2}^{(1)}\right)^{-1}\right\|_{h_{q}^{12} \rightarrow h_{q}^{12}} \\
& \leq\left(1+\varepsilon_{0}\right) \sup _{|j| \geq K_{2}}\left|\lambda_{j}\right| \\
& \leq\left(1+\varepsilon_{0}\right)\left(\frac{1}{2} K^{-c / y}\right) .
\end{aligned}
$$

Write

$$
\mathscr{B}=(k, \omega)\left(1+\frac{1}{ \pm(k, \omega)}\left(\Lambda_{2}^{(1)}+\breve{B}_{22}^{(11)}\right)\right) .
$$

Recall $|(k, \omega)| \geq K^{-c / y}$. By Neumann series and using (3.110),

$$
\left.\left\|\left\lfloor\mathscr{B}^{-1}\right\rceil\right\|_{h_{\tilde{p}}^{12} \rightarrow h_{\tilde{p}}^{12}} \leq K^{c / y} \sum_{j=0}^{\infty}\left(\| \frac{1}{|(k, \omega)|} L\left(\Lambda_{2}^{(1)}+\breve{B}_{22}^{(11)}\right)\right\rceil \|_{h_{\tilde{p}}^{12} \rightarrow h_{\tilde{p}}^{12}}\right)^{j} \leq 2 K^{c / y} .
$$

Since $U$ is unitary, so $\|U\|_{\ell_{2}^{12} \rightarrow \ell_{0}^{12}}=1$, and furthermore,

$$
\|U\|_{h_{\tilde{p}}^{12} \rightarrow h_{\tilde{p}}^{12}}=\left\|\left.\operatorname{diag}\left(|j|^{\tilde{p}}:|j| \leq K_{2}\right) U \operatorname{diag}\left(|j|^{-\tilde{p}}:|j| \leq K_{2}\right)\right|_{\ell_{2}^{12} \rightarrow \ell_{2}^{12}} \leq K_{2}^{\tilde{p}}|| U\right\|_{\ell_{2}^{12} \rightarrow \ell_{2}^{12}} \leq K^{3 c q / y} .
$$

By Lemma 12.4 moreover,

$$
\|U\|_{h_{\tilde{p}}^{12} \rightarrow h_{\tilde{p}}^{12}} \leq K^{3 c q / y} K_{2}^{d / 2}
$$

By (3.22) and noting $\mathscr{M}$ is diagonal,

$$
\left.\| L((k, \omega)+\mathscr{M})^{-1}\right\rceil\left\|_{\tilde{h}_{\tilde{p}}^{2} \rightarrow \tilde{h}_{\tilde{p}}^{2}}=\right\|((k, \omega)+\mathscr{M})^{-1} \|_{\tilde{h}_{\tilde{p}}^{2} \rightarrow \tilde{h}_{\tilde{p}}^{2}} \leq K^{c} .
$$

Consequently,

$$
\left\|\left\lfloor\mathscr{I}^{-1}\right\rceil\right\|_{h_{\tilde{p}, \tilde{p}}^{11}} \leq C\left(K^{c}+K^{c q / y}\right)^{3}<K_{5} \ll K_{3}
$$


Let $\pm=+$ without loss of generality in (3.106). Construct a Picard sequence as follows:

$$
\begin{array}{ll}
X_{0} & =Y \mathscr{I}^{-1} \\
X_{v+1} & \left.=X_{0}-\left(\left(\Lambda^{(2)}+B^{(22)}\right) X_{v}+\left(G_{22}\left(X_{v} \breve{B}^{(12)}\right)\right) \breve{B}^{(21)}\right)\right) \mathscr{I}^{-1}, \quad v=0,1,2, \ldots
\end{array}
$$

It follows

$$
\begin{aligned}
\left(\Lambda^{(2)}\right)^{-1} X_{v+1}= & \left(\Lambda^{(2)}\right)^{-1} Y \mathscr{I}^{-1} \\
& -\left(\left(1+\left(\Lambda^{(2)}\right)^{-1} B^{(22)}\right) \Lambda^{(2)}\left(\left(\Lambda^{(2)}\right)^{-1} X_{V}\right)\right) \mathscr{I}^{-1} \\
& +\left(\left(\left(\Lambda^{(2)}\right)^{-1}\left(G_{22}\left(X_{V} \breve{B}^{(12)}\right)\right)\right) \Lambda^{(2)}\left(\left(\Lambda^{(2)}\right)^{-1} \breve{B}^{(21)}\right)\right) \mathscr{I}^{-1}
\end{aligned}
$$

By (3.25),

$$
\left\|\left(\Lambda^{(2)}\right)^{-1}\left\lfloor B^{(22)}\right\rceil\right\|_{h_{p}^{2} \rightarrow h_{p}^{2}} \leq \varepsilon_{0},\left\|\left(\Lambda^{(2)}\right)^{-1}\left\lfloor\breve{B}^{(21)}\right\rceil\right\|_{h_{p}^{1} \rightarrow h_{p}^{2}} \leq \varepsilon_{0} .
$$

By (i) of this Lemma,

$$
\left\|\left(\Lambda^{(2)}\right)^{-1}\left\lfloor\left(G_{22}\left(X_{V} \breve{B}^{(12)}\right)\right)\right\rceil\right\|_{h_{p}^{2} \rightarrow h_{p}^{2}} \leq 2 K^{c}\left\|\left(\Lambda^{(2)}\right)^{-1}\left\lfloor X_{V} \breve{B}^{(12)}\right\rceil\right\|_{h_{p}^{2} \rightarrow h_{p}^{2}} \leq 2 K^{c} \varepsilon_{0}\left\|\left(\Lambda^{(2)}\right)^{-1}\left\lfloor X_{V}\right\rceil\right\|_{h_{p}^{1} \rightarrow h_{p}^{2}} .
$$

By the definition of $K_{3},\left\|\Lambda^{(2)}\right\|_{h_{p}^{2} \rightarrow h_{p}^{2}} \leq K_{3}^{-\kappa}$. Consequently

$$
\begin{aligned}
& \left\|\left(\Lambda^{(2)}\right)^{-1}\left\lfloor X_{V+1}\right\rceil-\left(\Lambda^{(2)}\right)^{-1}\left\lfloor X_{V}\right\rceil\right\|_{h_{p, p}^{12}} \\
\leq & \left(\left(1+\varepsilon_{0}\right) K_{5} K_{3}^{-\kappa}+2\right)\left\|\left(\Lambda^{(2)}\right)^{-1}\left\lfloor X_{V}\right\rceil-\left(\Lambda^{(2)}\right)^{-1}\left\lfloor X_{V-1}\right\rceil\right\|_{h_{p, p}^{12}} \\
\leq & \varepsilon_{0}\left\|\left(\Lambda^{(2)}\right)^{-1}\left\lfloor X_{V}\right\rceil-\left(\Lambda^{(2)}\right)^{-1}\left\lfloor X_{V-1}\right\rceil\right\|_{h_{p, p}^{12}} .
\end{aligned}
$$

It follows that $\left(\Lambda^{(2)}\right)^{-1} X_{V}$ is convergent to $\left(\Lambda^{(2)}\right)^{-1} X$ in the norm $\|L \cdot 7\|_{h_{p, p}^{11}}$, and

$$
\left\|\left(\Lambda^{(2)}\right)^{-1}\lfloor X\rceil\right\|_{h_{p}^{1} \rightarrow h_{p}^{2}} \leq K_{5}\left\|\left(\Lambda^{(2)}\right)^{-1}\lfloor Y\rceil\right\|_{h_{p}^{1} \rightarrow h_{p}^{2}} .
$$

Similarly,

$$
\left\|\lfloor X\rceil\left(\Lambda^{(1)}\right)^{-1}\right\|_{h_{q}^{1} \rightarrow h_{q}^{2}} \leq K_{5}\left\|\lfloor Y\rceil\left(\Lambda^{(1)}\right)^{-1}\right\|_{h_{q}^{1} \rightarrow h_{q}^{2}} .
$$

We omit the detail. This completes the proof of (ii).

Proof of (iii). The proof is similar to that of (ii). We omit it here.

Recall that

$$
\begin{gathered}
\left\|\Lambda^{-1}\lfloor R\rceil\right\|_{h_{p} \rightarrow h_{p}} \leq\|\lfloor R\rceil\|_{h_{p} \rightarrow h_{q}},\left\|\lfloor R\rceil \Lambda^{-1}\right\|_{h_{q} \rightarrow h_{q}} \leq\|R\|_{h_{p} \rightarrow h_{q}}, \\
\left\|\Lambda^{-1}\lfloor B\rceil\right\|_{h_{p} \rightarrow h_{p}} \leq\|B\|_{h_{p} \rightarrow h_{q}} \leq C \varepsilon_{0},\left\|\lfloor B\rceil \Lambda^{-1}\right\|_{h_{q} \rightarrow h_{q}} \leq\|\lfloor B\rceil\|_{h_{p} \rightarrow h_{q}} \leq C \varepsilon_{0}
\end{gathered}
$$

and

$$
N^{(21)}= \pm B^{(21)},\left\|\left\lfloor N^{(21)}\right\rceil\right\|_{h_{p}^{1} \rightarrow h_{p}^{2}} \leq\left\|\Lambda^{(2)}\right\|_{h_{p}^{2} \rightarrow h_{p}^{2}}\left\|\left(\Lambda^{(2)}\right)^{-1}\left\lfloor N^{(21)}\right\rceil\right\|_{h_{p}^{1} \rightarrow h_{p}^{2}} \leq \varepsilon_{0} K_{3}^{-\kappa} .
$$

By (i) of Lemma 3.5, one has

$$
\begin{aligned}
\left\|\left(\Lambda^{(2)}\right)^{-1}\left\lfloor G_{22}\left(R^{(22)} N^{(21)}\right)\right\rceil\right\|_{h_{p}^{1} \rightarrow h_{p}^{2}} & \leq K^{2 c}\left\|\left(\Lambda^{(2)}\right)^{-1}\left\lfloor R^{(22)}\right\rceil\right\|_{h_{p}^{2} \rightarrow h_{p}^{2}}\left\|\left\lfloor N^{(21)}\right\rceil\right\|_{h_{p}^{1} \rightarrow h_{p}^{2}} \\
& \leq \varepsilon_{0} K^{2 c} K_{3}^{-\kappa}\|\lfloor R\rceil\|_{h_{p} \rightarrow h_{q}} .
\end{aligned}
$$


By applying Lemma 3.5 to 3.38 , one has

$$
\begin{aligned}
& \left\|\left(\Lambda^{(2)}\right)^{-1}\left\lfloor\tilde{R}^{(21)}\right\rceil\right\|_{h_{p}^{1} \rightarrow h_{p}^{2}}=\left\|\left(\Lambda^{(2)}\right)^{-1} G_{21}\left(R^{(21)}-G_{22}\left(R^{(22)}\right) N^{(21)}\right)\right\|_{h_{p}^{1} \rightarrow h_{p}^{2}} \\
& \leq K_{5}\left\|\left\lfloor R^{(21)}-G_{22}\left(R^{(22)} N^{(21)}\right)\right\rceil\right\|_{h_{p}^{1} \rightarrow h_{q}^{2}} \leq K_{5}\left(\left\|\left\lfloor R^{(21)}\right\rceil\right\|_{h_{p}^{1} \rightarrow h_{q}^{2}}+\left\|\left\lfloor G_{22}\left(R^{(22)} N^{(21)}\right)\right\rceil\right\|_{h_{p}^{1} \rightarrow h_{q}^{2}}\right) \\
& =K_{5}\left\|\left\lfloor R^{(21)}\right\rceil\right\|_{h_{p}^{1} \rightarrow h_{q}^{2}}+K_{5}\left\|\left(\Lambda^{(2)}\right)^{-1}\left\lfloor G_{22}\left(R^{(22)} N^{(21)}\right)\right\rceil\right\|_{h_{p}^{1} \rightarrow h_{p}^{2}} \\
& \leq K_{5}\left\|\left\lfloor R^{(21)}\right\rceil\right\|_{h_{p}^{1} \rightarrow h_{q}^{2}}+K_{5} K^{c}\left\|\left(\Lambda^{(2)}\right)^{-1}\left\lfloor\left(R^{(22)} N^{(21)}\right)\right\rceil\right\|_{h_{p}^{1} \rightarrow h_{p}^{2}} \\
& \leq K_{5}\left\|\left\lfloor R^{(21)}\right\rceil\right\|_{h_{p}^{1} \rightarrow h_{q}^{2}}+K_{5} K^{c} \varepsilon_{0}\left\|\left\lfloor R^{(22)}\right\rceil\right\|_{h_{p}^{2} \rightarrow h_{p}^{2}} \\
& \leq 2 \varepsilon_{0} K^{c} K_{5}\|\lfloor R\rceil\|_{h_{p} \rightarrow h_{q}} .
\end{aligned}
$$

Moreover, applying Lemma 3.5 to (3.44), one has

$$
\begin{aligned}
& \left\|\left(\Lambda^{(1)}\right)^{-1}\left\lfloor\tilde{R}^{(12)}\right\rceil\right\|_{h_{p}^{2} \rightarrow h_{p}^{1}}=\left\|\left(\Lambda^{(1)}\right)^{-1}\left\lfloor G_{12}\left(M^{(12)}\left(G_{22}\left(R^{(22)}\right)-G_{22}\left(\tilde{R}^{(21)} N^{(12)}\right)\right)-R^{(12)}\right)\right\rceil\right\|_{h_{p}^{2} \rightarrow h_{p}^{1}} \\
& \leq K_{5}\left\|\left(\Lambda^{(1)}\right)^{-1}\left(M^{(12)}\left(G_{22}\left(R^{(22)}\right)-G_{22}\left(\tilde{R}^{(21)} N^{(12)}\right)\right)-R^{(12)}\right)\right\|_{h_{p}^{2} \rightarrow h_{p}^{1}} \\
& \leq K_{5}\left\|\left(\Lambda^{(1)}\right)^{-1}\left\lfloor M^{(12)}\right\rceil\right\|_{h_{p}^{2} \rightarrow h_{p}^{1}} \cdot\left\|\Lambda^{(2)}\right\|_{h_{p}^{2} \rightarrow h_{p}^{2}} \cdot\left\|\left(\Lambda^{(2)}\right)^{-1}\left\lfloor G_{22}\left(R^{(22)}-\left(\tilde{R}^{(21)} N^{(12)}\right)\right)\right\rceil\right\|_{h_{p}^{2} \rightarrow h_{p}^{2}} \\
& +K_{5}\left\|\left(\Lambda^{(1)}\right)^{-1} R^{(12)}\right\|_{h_{p}^{2} \rightarrow h_{p}^{1}} \\
& \leq K_{5} \varepsilon_{0} K_{3}^{-\kappa}\left\|\left(\Lambda^{(2)}\right)^{-1}\left\lfloor R^{(22)}-\tilde{R}^{(21)} N^{(12)}\right\rceil\right\|_{h_{p}^{2} \rightarrow h_{p}^{2}}+K_{5}\|\lfloor R\rceil\|_{h_{p} \rightarrow h_{q}} \\
& \leq K_{5} \varepsilon_{0} K_{3}^{-\kappa}\left(\left\|\left\lfloor R^{(22)}\right\rceil\right\|_{h_{p}^{2} \rightarrow h_{q}^{2}}+\left\|\left(\Lambda^{(2)}\right)^{-1}\left\lfloor\tilde{R}^{(21)}\right\rceil\right\|_{h_{p}^{1} \rightarrow h_{p}^{2}} \cdot \varepsilon_{0}\right)+K_{5}\|\lfloor R\rceil\|_{h_{p} \rightarrow h_{q}} \\
& \leq 2 K_{5}\|\lfloor R\rceil\|_{h_{p} \rightarrow h_{q}} .
\end{aligned}
$$

Finally, applying Lemma 3.5 to 3.48 , one has

$$
\left\|\left\lfloor\mathscr{R}^{(11)}\right\rceil\right\|_{h_{p, p}^{11}} \leq K_{5}^{2}\|\lfloor R\rceil\|_{h_{p} \rightarrow h_{q}} .
$$

Note that we can write $\mathscr{R}^{(11)}=\left(\mathscr{R}_{i j}^{(11)}:|i| \leq K_{3},|j| \leq K_{3}\right)$. So

$$
\left\|\operatorname{Vec} \mathscr{R}^{(11)}\right\|_{\ell_{2}^{1} \otimes \ell_{2}^{1}} \leq\left\|\operatorname{Vec} \mathscr{R}^{(11)}\right\|_{h_{p}^{1} \otimes h_{p}^{1}} \leq C K_{3}^{d} K_{5}^{2}\|\lfloor R\rceil\|_{h_{p} \rightarrow h_{q}} .
$$

By (3.102) and (3.117) to the equation (3.58), one gets

$$
\left\|\operatorname{Vec} F^{(11)}\right\|_{\ell_{2}^{1} \otimes \ell_{2}^{1}} \leq K^{C}||[R\rceil \|_{h_{p} \rightarrow h_{q}}
$$

where $C$ is a constant large enough such that $K^{C}>K_{3}^{d} K_{5}^{2}$. Noting that we can write $F^{(11)}=\left(F_{i j}^{(11)}:|i| \leq K_{3},|j| \leq\right.$ $K_{3}$ ), we have

$$
\left\|\left\lfloor F^{(11)}\right\rceil\right\|_{h_{p}^{1} \rightarrow h_{q}^{1}} \leq K^{5 C}\|\lfloor R\rceil\|_{h_{p} \rightarrow h_{q}} .
$$

By (3.118) and applying Lemma 3.5 to (3.37), (3.39) and (3.42), one has

$$
\|\lfloor\hat{F}(k)\rceil\|_{h_{p} \rightarrow h_{q}}=\left\|\left\lfloor\Lambda^{-1} \hat{F}(k)\right\rceil\right\|_{h_{p} \rightarrow h_{p}} \leq K^{5 C}||\lfloor\hat{R}(k)\rceil \|_{h_{p} \rightarrow h_{q}}, \forall 0<|k|<K .
$$

It follows (3.5) for $k \neq 0$. Applying $\partial_{\xi}$ to both sides of equation (3.3) and using (3.5), we can prove (3.6). Here we omit the detail.

Case 2. $k=0$. 
At this case, the sign \pm take + . So we can rewrite (3.10):

$$
(\Lambda+B) F+F(\Lambda+\breve{B})=R
$$

where $F:=\widehat{F}(0), R:=\widehat{R}(0)$. Consider the equation with the unknown variable $X$ :

$$
\Lambda X+X \Lambda=Y .
$$

Set

$$
g=(\Lambda(\cdot)+(\cdot) \Lambda)^{-1}
$$

Then $g(Y)=X$. Writing (3.121) in its elements of matrix

$$
X_{i j}=\frac{Y_{i j}}{\lambda_{i}+\lambda_{j}}, \quad i, j \in \mathbb{Z}^{d}
$$

Moreover,

$$
X_{i j}=\alpha_{i j} \tilde{Y}_{i j}=\beta_{i j} \breve{Y}_{i j}, \quad i, j \in \mathbb{Z}^{d}
$$

where

$$
\alpha_{i j}=\frac{\lambda_{i}}{\lambda_{i}+\lambda_{j}}, \beta_{i j}=\frac{\lambda_{j}}{\lambda_{i}+\lambda_{j}}, \quad \tilde{Y}_{i j}=\lambda_{i}^{-1} Y_{i j}, \breve{Y}_{i j}=Y_{i j} \lambda_{j}^{-1} .
$$

Note that $0<\alpha_{i j}<1$ and $0<\beta_{i j}<1$. Thus, $\left|X_{i j}\right| \leq\left|\tilde{Y}_{i j}\right|$ and $\left|X_{i j}\right| \leq\left|\breve{Y}_{i j}\right|$. Moreover,

$$
\|\lfloor g(Y)\rceil\|_{h_{p} \rightarrow h_{p}}=\mid\|\lfloor X\rceil\|_{h_{p} \rightarrow h_{p}} \leq\|\lfloor\tilde{Y}\rceil\|_{h_{p} \rightarrow h_{p}}=\|\lfloor Y\rceil\|_{h_{p} \rightarrow h_{q}}
$$

and

$$
\|\lfloor g(Y)\rceil\|_{h_{q} \rightarrow h_{q}}=\|\lfloor X\rceil\|_{h_{q} \rightarrow h_{q}} \leq\|\lfloor\breve{Y}\rceil\|_{h_{q} \rightarrow h_{q}}=\|\lfloor Y\rceil\|_{h_{p} \rightarrow h_{q}} .
$$

For an operator $X$ with $\lfloor X\rceil \in \mathscr{L}\left(h_{p} \rightarrow h_{p}\right)$ and $\lfloor X\rceil \in \mathscr{L}\left(h_{q} \rightarrow h_{q}\right)$, we call that $X$ is a $\tau$ - approximate solution of equation (3.120), if

$$
\|\lfloor(\Lambda+B) X+X(\Lambda+\breve{B})-R\rceil\|_{h_{p} \rightarrow h_{q}} \leq \tau .
$$

Let $X_{0}=g(R)$. By (3.125) and (3.126), we obtain

$$
\left\|\left\lfloor X_{0}\right\rceil\right\|_{h_{p} \rightarrow h_{p}} \leq\|\lfloor R\rceil\|_{h_{p} \rightarrow h_{q}} \triangleq \delta,\left\|\left\lfloor X_{0}\right\rceil\right\|_{h_{q} \rightarrow h_{q}} \leq\|\lfloor R\rceil\|_{h_{p} \rightarrow h_{q}}=\delta .
$$

Then

$$
\begin{aligned}
& \left\|\left\lfloor(\Lambda+B) X_{0}+X_{0}(\Lambda+\breve{B})-R\right\rceil\right\|_{h_{p} \rightarrow h_{q}}=\left\|\left\lfloor\left(\Lambda X_{0}+X_{0} \Lambda-R\right)+B X_{0}+X_{0} \breve{B}\right\rceil\right\|_{h_{p} \rightarrow h_{q}} \\
& =\left\|\left\lfloor B X_{0}+X_{0} \breve{B}\right\rceil\right\|_{h_{p} \rightarrow h_{q}} \leq\left\|\left\lfloor X_{0}\right\rceil\right\|_{h_{p} \rightarrow h_{p}} \cdot\|\lfloor B\rceil\|_{h_{p} \rightarrow h_{q}}+\|\lfloor\breve{B}\rceil\|_{h_{p} \rightarrow h_{q}} \cdot\left\|\left\lfloor X_{0}\right\rceil\right\|_{h_{q} \rightarrow h_{q}} \\
& \leq 2 \varepsilon_{0} \delta .
\end{aligned}
$$

That implies $X_{0}$ is a $2 \varepsilon_{0} \delta$ - approximate solution of (3.120). Let $X=X_{0}+Y$ and insert it into (3.120). Then

$$
(\Lambda+B) X_{0}+X_{0}(\Lambda+\breve{B})-R+\Lambda Y+Y \Lambda+B Y+Y \breve{B}=0 .
$$

Consider

$$
\Lambda Y+Y \Lambda=R-\left((\Lambda+B) X_{0}+X_{0}(\Lambda+\breve{B})\right):=R_{1} .
$$

Then

By (3.127), we have

$$
Y=g\left(R_{1}\right)
$$

$$
\|\lfloor Y\rceil\|_{h_{p} \rightarrow h_{q}} \leq 2 \varepsilon_{0} \delta, \quad\|\lfloor Y\rceil\|_{h_{q} \rightarrow h_{q}} \leq 2 \varepsilon_{0} \delta .
$$


Let $X_{1}=Y$ and $X^{(1)}=X_{0}+X_{1}$. By (3.128) and (3.130), we have

$$
\begin{aligned}
& \left\|\left\lfloor(\Lambda+B) X^{(1)}+X^{(1)}(\Lambda+\breve{B})-R\right\rceil\right\|_{h_{p} \rightarrow h_{q}}=\left\|\left\lfloor B X_{1}+X_{1} \breve{B}\right\rceil\right\|_{h_{p} \rightarrow h_{q}} \\
& \leq\left\|\left\lfloor X_{1}\right\rceil\right\|_{h_{p} \rightarrow h_{p}}\|\lfloor B\rceil\|_{h_{p} \rightarrow h_{q}}+\|\lfloor\breve{B}\rceil\|_{h_{p} \rightarrow h_{q}}\left\|\left\lfloor X_{1}\right\rceil\right\|_{h_{q} \rightarrow h_{q}} \leq\left(2 \varepsilon_{0}\right)^{2} \delta .
\end{aligned}
$$

Thus $X^{(1)}$ is $\left(2 \varepsilon_{0}\right)^{2} \delta$ - approximate solution of (3.120).

Assume that

$$
X^{(m)}=X_{0}+X_{1}+\cdots+X_{m-1}
$$

is a $\left(2 \varepsilon_{0}\right)^{m+1} \delta$ - approximate solution of (3.120). Thus

$$
R_{m}:=R-\left((\Lambda+B) X^{(m)}+X^{(m)}(\Lambda+\breve{B})\right)
$$

satisfies

$$
\left\|\left\lfloor R_{m}\right\rceil\right\|_{h_{p} \rightarrow h_{q}} \leq\left(2 \varepsilon_{0}\right)^{m+1} \delta .
$$

Take $X_{m}=g\left(R_{m}\right)$. That means $\Lambda X_{m}+X_{m} \Lambda=R_{m}$. Then

$$
\begin{aligned}
& \left\|\left\lfloor X_{m}\right\rceil\right\|_{h_{p} \rightarrow h_{p}} \leq\left\|\left\lfloor R_{m}\right\rceil\right\|_{h_{p} \rightarrow h_{q}} \leq\left(2 \varepsilon_{0}\right)^{m+1} \delta, \\
& \left.\left\|\left\lfloor X_{m}\right\rceil\right\|\right|_{h_{q} \rightarrow h_{q}} \leq\left\|\left\lfloor R_{m}\right\rceil\right\|_{h_{p} \rightarrow h_{q}} \leq\left(2 \varepsilon_{0}\right)^{m+1} \delta .
\end{aligned}
$$

Let

$$
X^{(m+1)}=X_{0}+X_{1}+\cdots+X_{m-1}+X_{m}=X^{(m)}+X_{m} .
$$

Then It follows (3.131) that

$$
\begin{aligned}
& \left\|\left\lfloor(\Lambda+B) X^{(m+1)}-X^{(m+1)}(\Lambda+\breve{B})-R_{m}\right\rceil\right\|_{h_{p} \rightarrow h_{q}} \\
& =\left\|\left\lfloor\Lambda X_{m}-X_{m} \Lambda-R_{m}+B X_{m}+X_{m} \breve{B}\right\rceil\right\|_{h_{p} \rightarrow h_{q}} \\
& =\left\|\left\lfloor B X_{m}+X_{m} \breve{B}\right\rceil\right\|_{h_{p} \rightarrow h_{q}} \leq\left\|\left\lfloor X_{m}\right\rceil\right\|_{h_{p} \rightarrow h_{p}}\|\lfloor B\rceil\|_{h_{p} \rightarrow h_{q}}+\|\lfloor\breve{B}\rceil\|_{h_{p} \rightarrow h_{q}}\left\|\left\lfloor X_{m}\right\rceil\right\|_{h_{q} \rightarrow h_{q}} \\
& \leq\left(2 \varepsilon_{0}\right)^{m+2} \delta .
\end{aligned}
$$

Thus $X^{(m+1)}$ is a $\left(2 \varepsilon_{0}\right)^{m+2} \delta$ - approximate solution.

By induction, we can assume

$$
\begin{aligned}
& \sum_{m=0}^{\infty} X_{m}=X, \text { in }\|\cdot\|_{h_{p} \rightarrow h_{p}}, \\
& \sum_{m=0}^{\infty} X_{m}=\tilde{X}, \text { in }\|\cdot\|_{h_{q} \rightarrow h_{q}} .
\end{aligned}
$$

For any $L>0$ and $M=\left(m_{i j} \in \mathbb{C}: i, j \in \mathbb{Z}^{d}\right)$, define

$$
M^{L}=\left(m_{i j} \in \mathbb{C}:|i| \leq L,|j| \leq L\right) .
$$

Similarly, we can define $h_{p}^{L}$ and $h_{q}^{L}$. By Lemma12.1, we have

$$
\begin{aligned}
& \sum_{m=0}^{\infty} X_{m}^{L}=X^{L}, \text { in }\|\cdot\|_{h_{p}^{L} \rightarrow h_{p}^{L}}, \\
& \sum_{m=0}^{\infty} X_{m}^{L}=\widetilde{X}^{L}, \text { in }\|\cdot\|_{h_{q}^{L} \rightarrow h_{q}^{L}} .
\end{aligned}
$$


By $\operatorname{dim} X^{L}<\infty$ and $\operatorname{dim} \widetilde{X}^{L}<\infty$, we have $X^{L}=\widetilde{X}^{L}$. Since $L>0$ is arbitrary, we have $X=\widetilde{X}=\widehat{F}(0, \xi)$. Therefore,

$$
X=\sum_{m=0}^{\infty} X_{m}
$$

solves $(\underline{3.120}$ ) and

$$
\|\lfloor X\rceil\|_{h_{p} \rightarrow h_{p}} \leq \delta+\sum_{m=0}^{\infty}\left(2 \varepsilon_{0}\right)^{m+1} \delta \leq 2 \delta, \quad\|\lfloor X\rceil\|_{h_{q} \rightarrow h_{q}} \leq \delta+\sum_{m=0}^{\infty}\left(2 \varepsilon_{0}\right)^{m+1} \delta \leq 2 \delta .
$$

This completes the proof of (3.7). Applying $\partial_{\xi}$ to both sides of (3.120), we can prove (3.8) similarly.

Up to now, the proof of Lemma 3.1 is completed.

Remark 9. Comparing (3.132) and (3.119), we find that $\widehat{F}(k)$ with $k \neq 0$ is of regularity of order $\kappa$, while $\widehat{F}(0)$ has no regularity.

\section{Iterative Lemma}

Before giving the iterative lemma, we need the following iterative constants and domains:

- $m$ - number of the iterative steps;

- $C, C_{1}, C_{2}, \ldots$ - positive constants which arrive in estimates. They are independent of $\varepsilon_{0}$ and $m$, maybe different in different position of the text;

- $\varepsilon_{m}=\varepsilon_{0}^{\left(1+\rho_{0}\right)^{m}}$, which measures the size of the perturbation in the $m^{\text {th }}$ iteration, $m=1,2, \ldots$, where $\rho_{0}>0$ is an absolute constant;

- $e_{m}=\frac{1^{-2}+2^{-2}+\cdots+m^{-2}}{2\left(1^{-2}+2^{-2}+\cdots\right)}\left(\right.$ so $0<e_{m}<\frac{1}{2}$ for all $\left.m\right)$;

- $s_{m}=s_{0}\left(1-e_{m}\right)$ (so $s_{m}>\frac{1}{2} s_{0}$ for all $\left.m\right)$, which measure the width of the angle variable $x$ in the $m^{\text {th }}$ iteration, here $s_{0}>0$ is an absolute constant;

- $r_{m}=r_{0}\left(1-e_{m}\right)$ (so $r_{m}>\frac{1}{2} r_{0}$ for all $m$ ), which measure the radius of the action variable $y$ as well as the normal coordinate $(z, \bar{z})$ in the $m^{\text {th }}$ iteration, $r_{0}>0$ is an absolute constant;

- $s_{m}^{j}=\left(1-\frac{j}{6}\right) s_{m}+\frac{j}{6} s_{m+1}, \quad(j=0,1, \ldots, 6)$ which is a bridge between $s_{m}$ and $s_{m+1}$;

- $r_{m}^{j}=\left(1-\frac{j}{6}\right) r_{m}+\frac{j}{6} r_{m+1},(j=0,1, \ldots, 6)$ which is a bridge between $r_{m}$ and $r_{m+1}$;

- $K_{m}=\frac{2}{s_{m}^{5}-s_{m}^{b}}\left|\log \varepsilon_{m}\right|$ which truncates a periodic function into essential part and unessential one in the term of its Fourier coefficient;

- $C(m), C_{1}(m), \ldots$ functions of $m$ and of the form $C_{1} m^{C_{2}}$ or $C_{1} 2^{C m}$;

- $D_{p}\left(s_{m}, r_{m}\right)=\left\{(x, y, z, \bar{z}) \in \mathscr{P}^{p}:|\mathfrak{I} x|<s_{m},|y|<r_{m}^{2},\|z\|_{p}<r_{m},\|\bar{z}\|_{p}<r_{m}\right\}$, which denotes a complexificated neighborhood of the torus

$$
\mathscr{T}_{0}:=\mathbb{T}^{N} \times\{0\} \times\{0\} \times\{0\} \subset \mathscr{P}^{p} .
$$

Obviously,

$$
D_{p}\left(s_{0}, r_{0}\right) \supset D\left(s_{1}, r_{1}\right) \supset \cdots \supset D_{p}\left(s_{m}, r_{m}\right) \supset \cdots \supset D_{p}\left(\frac{s_{0}}{2}, \frac{r_{0}}{2}\right) ;
$$


- $\mathbb{T}_{s_{m}}^{N}=\left\{x \in \mathbb{C}^{n} /(2 \pi \mathbb{Z})^{n}:|\mathfrak{I} x| \leq s_{m}\right\}$, which is a complixificated neighborhood of $\mathbb{T}^{N}$ with strip width $s_{m}$. Obviously,

$$
\mathbb{T}_{s_{0}}^{N} \supset \mathbb{T}_{s_{1}}^{N} \supset \cdots \supset \mathbb{T}_{s_{m}}^{N} \supset \cdots \supset \mathbb{T}_{s_{0} / 2}^{N}
$$

The proof of Theorem 1.1 will be completed by $m \rightarrow+\infty$ in the following lemma:

Lemma 4.1. Let $\omega^{0}, \lambda_{j}$ 's and $B^{0}$ obey the assumptions $\mathbf{A}, \mathbf{B}$ and $\mathbf{E}$ in Theorem 1.1 respectively. Suppose that we have had $m+1$ Hamiltonian functions

$$
H^{(l)}=H_{0}^{(l)}+R^{(l)}+P^{(l)}, \quad l=0,1, \cdots, m,
$$

where

$$
H_{0}^{(l)}=\left(\omega^{(l)}(\xi), y\right)+\sum_{j \in \mathbb{Z}^{d}} \lambda_{j}(\xi) z_{j} \bar{z}_{j}+\left\langle B^{(l) z \bar{z}}(\xi) z, \bar{z}\right\rangle .
$$

And suppose that there are $m+1$ closed parameters sets

$$
\mathbb{R}^{N} \supset \mathscr{O}_{0} \supset \mathscr{O}_{1} \supset \cdots \supset \mathscr{O}_{m}
$$

and $m+1$ domains

$$
D_{p}\left(s_{0}, r_{0}\right) \supset \cdots \supset D_{p}\left(s_{l}, r_{l}\right) \supset \cdots D_{p}\left(s_{m}, r_{m}\right) \supset D_{p}\left(s_{0} / 2, r_{0} / 2\right)
$$

such that

$(1)_{l}$

$$
\omega^{(l)}=\omega^{0}+\sum_{j=1}^{l} \omega_{(j)}, \quad l=1,2, \cdots, m, \quad \omega^{(0)}=\omega^{0},
$$

where the function $\omega_{(j)}=\omega_{(j)}(\xi): \mathscr{O}_{j} \rightarrow \mathbb{R}^{N}$ is smooth, and

$$
\sup _{\xi \in \mathscr{O}_{j}}\left|\omega_{(j)}\right| \leq C(j-1) \varepsilon_{j-1}, \quad \sup _{\xi \in \mathscr{O}_{j}}\left|\partial_{\xi} \omega_{(j)}\right| \leq C(j-1) \varepsilon_{j-1}, \quad j=1, \cdots, l ;
$$

$$
B^{(l) z \bar{z}}=\sum_{j=0}^{l} B_{(j)}^{z \bar{z}}, \quad j=1, \cdots, l, \quad B_{(0)}^{z \bar{z}}=B^{0},
$$

where the operator-value functions $\left\lfloor B_{(j)}^{z \bar{z}}\right\rceil=\left\lfloor B_{(j)}^{z \bar{z}}(\xi)\right\rceil: \mathscr{O}_{j} \rightarrow \mathscr{L}\left(h_{p}, h_{q}\right)$ is smooth in $\xi \in \mathscr{O}_{j}$ with

$$
\begin{gathered}
\sup _{\xi \in \mathscr{O}_{j}}\left\|\left\lfloor B_{(j)}^{z \bar{z}}(\xi)\right\rceil\right\|_{h_{p} \rightarrow h_{q}} \leq C(j-1) \varepsilon_{j-1}, j=1, \ldots, l, \\
\sup _{\xi \in \mathscr{O}_{j}}\left\|\left\lfloor\partial_{\xi}\left(B_{(j)}^{z \bar{z}}(\xi)\right)\right\rceil\right\|_{h_{p} \rightarrow h_{q}} \leq C(j-1) \varepsilon_{j-1}, j=1, \ldots, l ;
\end{gathered}
$$

(3) $)_{l}$ For $l=0,1, \ldots, m$, the vector fields $X_{R^{(l)}}: D_{p}\left(s_{l}, r_{l}\right) \times \mathscr{O}_{l} \subset \mathscr{P}^{p} \times \mathscr{O}_{l} \rightarrow \mathscr{P}^{q}$ are analytic in $(x, y, z, \bar{z}) \in D_{p}\left(s_{l}, r_{l}\right)$ for fixed $\xi \in \mathscr{O}_{l}$ and smooth in $\mathscr{O}_{l}$ for fixed $(x, y, z, \bar{z}) \in D_{p}\left(s_{l}, r_{l}\right)$, and

$$
\left|\left\lfloor X_{R^{(l)}}\right\rceil\right|_{q, D_{p}\left(s_{l}, r_{l}\right) \times \mathscr{O}_{l}} \leq C(l) \varepsilon_{l},\left|\left\lfloor\partial_{\xi} X_{R^{(l)}}\right\rceil\right|_{q, D_{p}\left(s_{l}, r_{l}\right) \times \mathscr{O}_{l}} \leq C(l) \varepsilon_{l} ;
$$

(4) $)_{l}$ For $l=0,1, \ldots, m$, the vector fields $X_{P^{(l)}}: D_{p}\left(s_{l}, r_{l}\right) \times \mathscr{O}_{l} \subset \mathscr{P}^{p} \times \mathscr{O}_{l} \rightarrow \mathscr{P}^{q}$ analytic in $(x, y, z, \bar{z}) \in D_{p}\left(s_{l}, r_{l}\right)$ for fixed $\xi \in \mathscr{O}_{l}$ and smooth in $\mathscr{O}_{l}$ for fixed $(x, y, z, \bar{z}) \in D_{p}\left(s_{l}, r_{l}\right)$, and

$$
P^{(l)}=O\left(|y|^{2}+|y||| z||_{p}+|| z||_{p}^{3}\right)
$$


and

$$
\left|\left\lfloor X_{P^{(l)}}\right\rceil\right|_{q, D_{p}\left(s_{l}, r_{l}\right) \times \mathscr{O}_{l}} \leq C,\left|\left\lfloor\partial_{\xi} X_{P^{(l)}}\right\rceil\right|_{q, D_{p}\left(s_{l}, r_{l}\right) \times \mathscr{O}_{l}} \leq C ;
$$

$(5)_{l}$ The Hamiltonian functions $H_{0}^{(l)}$ and $R^{(l)}$ and $P^{(l)}$ are real when $(x, y)$ are real and $\bar{z}$ is the complex conjugate of $z$, for $(x, y, z, \bar{z} ; \xi) \in D_{p}\left(s_{l}, r_{l}\right) \times \mathscr{O}_{l}$.

Then there exists a subset $\mathscr{O}_{m+1} \subset \mathscr{O}_{m}$ with

$$
\text { Meas } \mathscr{O}_{m+1}=\left(\text { Meas } \mathscr{O}_{m}\right)\left(1-O\left(K_{m}^{-C}\right)\right)
$$

and a symplectic transformation

$$
\Psi_{m}: D_{p}\left(s_{m+1}, r_{m+1}\right) \times \mathscr{O}_{m+1} \rightarrow D_{p}\left(s_{m}, r_{m}\right) \times \mathscr{O}_{m}
$$

such that

$$
H_{m+1}:=H_{m} \circ \Psi=N^{(m+1)}+R^{(m+1)}+P^{(m+1)}
$$

satisfies the above conditions $(1)_{l}-(5)_{l}$ with $l=m+1$.

\section{Derivation of homological equations}

Step 1: Splitting the perturbation.

In the iterative lemma, the step number $l=0,1, \cdots, m$. Consider the perturbation $R^{(l)}$ with $l=m$. Decompose $R^{(m)}$ into

$$
R^{(m)}=R^{(2 m)}+R^{(3 m)}
$$

with

$$
\begin{aligned}
R^{(2 m)}= & R^{x}(x, \xi)+\left(R^{y}(x, \xi), y\right) \\
& +\left\langle R^{z}(x, \xi), z\right\rangle+\left\langle R^{\bar{z}}(x, \xi), \bar{z}\right\rangle \\
& +\left\langle R^{z z}(x, \xi) z, z\right\rangle+\left\langle R^{z \bar{z}}(x, \xi) z, \bar{z}\right\rangle+\left\langle R^{\bar{z}}(x, \xi) \bar{z}, \bar{z}\right\rangle
\end{aligned}
$$

and

$$
R^{(3 m)}=R^{(m)}-R^{(2 m)}=O\left(|y|^{2}+|y||| z||_{p}+\|z\|_{p}^{3}\right),
$$

where we can assume $\widehat{R}^{x}(0, \xi)$, the 0-Fourier coefficient of $R^{x}(x, \xi)$, vanishes, since it does not affect the dynamics.

Lemma 5.1. Let $u, v \in\{z, \bar{z}\}$. The terms $R^{x}(x, \xi), R^{y}(x, \xi), R^{u}(x, \xi)$ and $R^{u v}(x, \xi)$ are analytic in $x \in \mathbb{T}_{s_{m}}^{N}$ for fixed $\xi$ and smooth in $\xi \in \mathscr{O}_{m}$ for fixed $x$, and obey the following estimates

(i)

$$
\begin{aligned}
& \left|\left\lfloor R^{x}\right\rceil\right|_{s_{m}, \mathscr{O}_{m}} \leq C(m) \varepsilon_{m}, \quad\left|\left\lfloor\partial_{\xi} R^{x}\right\rceil\right|_{s_{m}, \mathscr{O}_{m}} \leq C(m) \varepsilon_{m}, \\
& \left|\left\lfloor R^{y}\right\rceil\right|_{s_{m}, \mathscr{O}_{m}} \leq C(m) \varepsilon_{m}, \quad\left|\left\lfloor\partial_{\xi} R^{y}\right\rceil\right|_{s_{m}, \mathscr{O}_{m}} \leq C(m) \varepsilon_{m},
\end{aligned}
$$

(ii)

$$
\left\|\left\lfloor R^{u}\right\rceil\right\|_{q, s_{m}, \mathscr{O}_{m}} \leq C(m) \varepsilon_{m}, \quad\left\|\left\lfloor\partial_{\xi} R^{u}\right\rceil\right\|_{q, s_{m}, \mathscr{O}_{m}} \leq C(m) \varepsilon_{m}, u \in\{z, \bar{z}\}
$$

(iii) for $u, v \in\{z, \bar{z}\}$,

$$
\sup _{\mathbb{T}_{s_{m}}^{N} \times \mathscr{O}_{m}}\left\|\left\lfloor R^{u v}(x, \xi)\right\rceil\right\|_{h_{p} \rightarrow h_{q}} \leq C(m) \varepsilon_{m}, \sup _{\mathbb{T}_{s_{m}}^{N} \times \mathscr{O}_{m}}\left\|\left\lfloor\partial_{\xi} R^{u v}(x, \xi)\right\rceil\right\|_{h_{p} \rightarrow h_{q}} \leq C(m) \varepsilon_{m} .
$$


(iv) The perturbation $R^{(3 m)}$ is analytic in $(x, y, z, \bar{z}) \in D_{p}\left(s_{m}, r_{m}\right)$ for fixed $\xi$ and smooth in $\xi \in \mathscr{O}_{m}$ for fixed $(x, y, z, \bar{z}) \in D_{p}\left(s_{m}, r_{m}\right)$, and real when $x$ and $y$ are real and $\bar{z}$ is the complex conjugate of $z$, and obeys the following estimates

$$
\left.\mid\left\lfloor X_{R^{(3 m)}}\right\rceil\right\rceil_{q, D_{p}\left(s_{m}, r_{m}\right) \times \mathscr{O}_{m}} \leq C(m), \quad\left|\left\lfloor\partial_{\xi} X_{R^{(3 m)}}\right\rceil\right|_{q, D_{p}\left(s_{m}, r_{m}\right) \times \mathscr{O}_{m}} \leq C(m) .
$$

Proof. The proofs for (5.4), (5.5) and (5.6) and (5.8) are trivial or simpler than that of (5.7). We give only the proof for the first inequality in (5.7) with $u=z, v=\bar{z}$. Note that

$$
\left\lfloor R^{z \bar{z}}(x, \xi)\right\rceil=\left.\left(\partial_{z}\left\lfloor\partial_{\bar{z}} R^{(m)}\right\rceil\right)\right|_{z=\bar{z}=0},
$$

where $\left\lfloor\partial_{\bar{z}} R^{(m)}\right\rceil$ is one entry of the modulus $\left\lfloor X_{R^{(m)}}\right\rceil$. By Cauchy's estimate,

$$
\sup _{\mathbb{T}_{s_{m}}^{N} \times \mathscr{O}_{m}}\left\|\left\lfloor R^{z \bar{z}}(x, \xi)\right\rceil\right\|_{h_{p} \rightarrow h_{q}} \leq \frac{1}{r_{m}}\left|\left\lfloor X_{R^{(m)}}\right\rceil\right|_{q, D_{p}\left(s_{m}, r_{m}\right) \times \mathscr{O}_{m}} \leq \frac{1}{r_{m}} \varepsilon_{m} \leq \frac{4}{r_{0}} \varepsilon_{m} \leq C(m) \varepsilon_{m},
$$

where (4.10) is used in the second inequality.

Step 2. New form of Hamiltonian $H^{(m)}$. Let

$$
\omega_{m}^{-}:=\widehat{R^{y}}(0, \xi) .
$$

By (5.5),

$$
\sup _{\xi \in \mathscr{O}_{m}}\left|\omega_{m}^{-}(\xi)\right| \leq C(m) \varepsilon_{m}, \quad \sup _{\xi \in \mathscr{O}_{m}}\left|\partial_{\xi} \omega_{m}^{-}(\xi)\right| \leq C(m) \varepsilon_{m} .
$$

Let

$$
\begin{gathered}
\widetilde{\omega}^{(m+1)}=\omega^{(m)}+\omega_{m}^{-}, \\
\tilde{B}^{(m+1)}=B^{(m)}+\widehat{R^{z z}}(0, \xi) .
\end{gathered}
$$

And set

$$
\begin{aligned}
\tilde{H}_{0}^{(m+1)}= & \left(\tilde{\omega}^{(m+1)}, y\right)+\langle\Lambda z, \bar{z}\rangle+\left\langle\tilde{B}^{(m+1)}(\xi) z, \bar{z}\right\rangle, \\
& R_{*}^{y}(x, \xi)=R^{y}(x, \xi)-\widehat{R^{y}}(0, \xi) \\
& R_{*}^{z \bar{z}}(x, \xi)=R^{z \bar{z}}(x, \xi)-\widehat{R^{z \bar{z}}}(0, \xi)
\end{aligned}
$$

and

$$
\begin{aligned}
R_{*}^{(2 m)}= & R^{x}(x, \xi)+\left(R_{*}^{y}(x, \xi), y\right) \\
& +\left\langle R^{z}(x, \xi), z\right\rangle+\left\langle R^{\bar{z}}(x, \xi), \bar{z}\right\rangle \\
& +\left\langle R^{z z}(x, \xi) z, z\right\rangle+\left\langle R_{*}^{z \bar{z}}(x, \xi) z, \bar{z}\right\rangle+\left\langle R^{\bar{z}}(x, \xi) \bar{z}, \bar{z}\right\rangle,
\end{aligned}
$$

where $\widehat{R}^{x}(0, \xi)=0, \widehat{R_{*}^{y}}(0, \xi)=0$ and $\widehat{R_{*}^{z \bar{z}}}(0, \xi)=0$.

Consequently, the Hamiltonian $H^{(m)}$ takes on a new form

$$
H^{(m)}=\tilde{H}_{0}^{(m+1)}+R_{*}^{(2 m)}+R^{(3 m)}+P^{(m)} .
$$

Step 3. Derivation of homological equations. Suppose the to-be-specified Hamiltonian $F$ is of the same form as of $R_{*}^{(2 m)}$ :

$$
\begin{aligned}
F= & F^{x}(x, \xi)+\left(F^{y}(x, \xi), y\right) \\
& +\left\langle F^{z}(x, \xi), z\right\rangle+\left\langle F^{\bar{z}}(x, \xi), \bar{z}\right\rangle \\
& +\left\langle F^{z z}(x, \xi) z, z\right\rangle+\left\langle F^{z \bar{z}}(x, \xi) z, \bar{z}\right\rangle+\left\langle F^{\bar{z} \bar{z}}(x, \xi) \bar{z}, \bar{z}\right\rangle
\end{aligned}
$$


with

$$
\widehat{F^{x}}(0, \xi)=0, \widehat{F^{y}}(0, \xi)=0, \widehat{F^{z \bar{z}}}(0, \xi)=0
$$

and it is expected that

$$
F=O\left(\varepsilon_{m}\right)
$$

Let

$$
H^{(m+1)}=\left.H^{(m)} \circ X_{F}^{t}\right|_{t=1}
$$

where $X_{F}^{t}$ is the flow of Hamiltonian vector field $X_{F}$ with Hamiltonian $F$ with the symplectic structure (1.2). By Taylor's formula,

$$
\begin{aligned}
H^{m+1}= & H^{(m)}+\left\{H^{(m)}, F\right\}+\int_{0}^{1} \int_{0}^{t}\left\{\left\{H^{(m)}, F\right\}, F\right\} \circ X_{F}^{\tau} d \tau d t \\
= & \tilde{H}_{0}^{(m+1)}+R_{*}^{(2 m)}+R^{(3 m)}+P^{(m)}+\left\{\tilde{H}_{0}^{(m+1)}, F\right\}+\left\{R_{*}^{(2 m)}, F\right\} \\
& +\left\{R^{(3 m)}+P^{(m)}, F\right\}+\int_{0}^{1} \int_{0}^{t}\left\{\left\{H^{(m)}, F\right\}, F\right\} \circ X_{F}^{\tau} d \tau d t,
\end{aligned}
$$

where $\{\cdot, \cdot\}$ is the Poisson bracket with the symplectic structure (1.2), that is, for any two Hamiltonian functions $f$ and $g$ of $(x, y, z, \bar{z})$,

$$
\{f, g\}=\partial_{x} f \cdot \partial_{y} g-\partial_{y} g \cdot \partial_{x} f+\mathbf{i}\left\langle\partial_{z} f, \partial_{z} g\right\rangle-\mathbf{i}\left\langle\partial_{z} f, \partial_{z} g\right\rangle .
$$

Set

$$
\tilde{H}_{0}:=\tilde{H}_{0}^{(m+1)}, \quad B=\tilde{B}^{(m+1)}, \quad \omega=\tilde{\omega}^{(m+1)} .
$$

By calculation,

$$
\begin{aligned}
\left\{\tilde{H}_{0}, F\right\}= & \partial_{x} \tilde{H}_{0} \cdot \partial_{y} F-\partial_{y} \tilde{H}_{0} \cdot \partial_{x} F-\mathbf{i}\left\langle\partial_{\bar{z}} \tilde{H}_{0}, \partial_{z} F\right\rangle+\mathbf{i}\left\langle\partial_{z} \tilde{H}_{0}, \partial_{\bar{z}} F\right\rangle \\
= & -\omega \cdot \partial_{x} F^{x}-\left(\omega \cdot \partial_{x} F^{y}, y\right)-\left\langle\omega \cdot \partial_{x} F^{z}, z\right\rangle-\left\langle\omega \cdot \partial_{x} F^{\bar{z}}, \bar{z}\right\rangle \\
& -\left\langle\omega \cdot \partial_{x} F^{z z} z, z\right\rangle-\left\langle\omega \cdot \partial_{x} F_{\bar{z} \bar{z}} \bar{z}, \bar{z}\right\rangle-\left\langle\omega \cdot \partial_{x} F^{z \bar{z}} z, \bar{z}\right\rangle \\
& +\mathbf{i}\left(\left\langle(\Lambda+B) F^{\bar{z}}, \bar{z}\right\rangle+\left\langle(\Lambda+B) F^{z \bar{z}} z, \bar{z}\right\rangle+\left\langle(\Lambda+B) F^{\bar{z}} \bar{z} \bar{z}, \bar{z}\right\rangle+\left\langle F^{\bar{z} z}\left(\Lambda+B^{T}\right) \bar{z}, \bar{z}\right\rangle\right) \\
& -\mathbf{i}\left(\left\langle\left(\Lambda+B^{T}\right) F^{z}, z\right\rangle+\left\langle F^{z \bar{z}}(\Lambda+B) z, \bar{z}\right\rangle+\left\langle\left(\Lambda+B^{T}\right) F^{z z} z, z\right\rangle+\left\langle F^{z z}(\Lambda+B) z, z\right\rangle\right),
\end{aligned}
$$

where $T$ denotes the transpose of matrix. By Taylor's formula, decompose $\left\{R^{(3 m)}+P^{(m)}, F\right\}$ into the lower order terms $R_{+}^{(2 m)}$ and the higher order terms $R_{+}^{(3 m)}$ :

$$
\left\{R^{(3 m)}+P^{(m)}, F\right\}=R_{+}^{(2 m)}+R_{+}^{(3 m)},
$$

where

$$
\begin{aligned}
R_{+}^{(2 m)}= & R_{+}^{x}(x, \xi)+\left(R_{+}^{y}(x, \xi), y\right)+ \\
& \left\langle R_{+}^{z}(x, \xi), z\right\rangle+\left\langle R_{+}^{\bar{z}}(x, \xi), \bar{z}\right\rangle+ \\
& \left\langle R_{+}^{z z}(x, \xi) z, z\right\rangle+\left\langle R_{+}^{\bar{z}}(x, \xi) \bar{z}, \bar{z}\right\rangle+ \\
& \left\langle R_{+}^{z \bar{z}}(x, \xi) z, \bar{z}\right\rangle, \\
R_{+}^{(3 m)}= & O\left(|y|^{2}+|y|\|z\|_{p}+\|z\|_{p}^{3}\right) .
\end{aligned}
$$

Noting that

$$
\tilde{P}^{(m)}:=R^{(3 m)}+P^{(m)}=O\left(|y|^{2}+\left.|y||| z\left|\left\|_{p}+\right\| z\right|\right|_{p} ^{3}\right) .
$$

By calculation, we have

$$
R_{+}^{x}(x, \xi) \equiv 0,
$$




$$
\begin{gathered}
R_{+}^{y}(x, \xi)=-\left.\partial_{y}\left(\partial_{y} \tilde{P}^{(m)} \cdot \partial_{x} F^{x}-\mathbf{i}\left\langle\partial_{\bar{z}} \tilde{P}^{(m)}, F^{z}\right\rangle+\mathbf{i}\left\langle\partial_{z} \tilde{P}^{(m)}, F^{\bar{z}}\right\rangle\right)\right|_{y=0, z=\bar{z}=0}, \\
R_{+}^{z}(x, \xi)=-\left.\partial_{z}\left(\partial_{y} \tilde{P}^{(m)}, \partial_{x} F^{x}\right)\right|_{y=0, z=\bar{z}=0}, \\
R_{+}^{\bar{z}}(x, \xi)=-\left.\partial_{\bar{z}}\left(\partial_{y} \tilde{P}^{(m)}, \partial_{x} F^{x}\right)\right|_{y=0, z=\bar{z}=0}, \\
R_{+}^{z z}(x, \xi)=\left.\partial_{z} \partial_{z}\left(\partial_{y} \tilde{P}^{(m)} \cdot \partial_{x}\left(\left\langle F^{z}, z\right\rangle+\left\langle F^{\bar{z}}, \bar{z}\right\rangle\right)+\mathbf{i}\left\langle\partial_{z} \tilde{P}^{(m)}, F^{\bar{z}}\right\rangle-\mathbf{i}\left\langle\partial_{\bar{z}} \tilde{P}^{(m)}, F^{z}\right\rangle\right)\right|_{y=0, z=\bar{z}=0}, \\
R_{+}^{\bar{z} \bar{z}}(x, \xi)=\left.\partial_{\bar{z}} \partial_{\bar{z}}\left(\partial_{y} \tilde{P}^{(m)} \cdot \partial_{x}\left(\left\langle F^{z}, z\right\rangle+\left\langle F^{\bar{z}}, \bar{z}\right\rangle\right)+\mathbf{i}\left\langle\partial_{z} \tilde{P}^{(m)}, F^{\bar{z}}\right\rangle-\mathbf{i}\left\langle\partial_{\bar{z}} \tilde{P}^{(m)}, F^{z}\right\rangle\right)\right|_{y=0, z=\bar{z}=0}, \\
R_{+}^{z \bar{z}}(x, \xi)=\left.\partial_{z} \partial_{\bar{z}}\left(\partial_{y} \tilde{P}^{(m)} \cdot \partial_{x}\left(\left\langle F^{z}, z\right\rangle+\left\langle F^{\bar{z}}, \bar{z}\right\rangle\right)+\mathbf{i}\left\langle\partial_{z} \tilde{P}^{(m)}, F^{\bar{z}}\right\rangle-\mathbf{i}\left\langle\partial_{\bar{z}} \tilde{P}^{(m)}, F^{z}\right\rangle\right)\right|_{y=0, z=\bar{z}=0} .
\end{gathered}
$$

Let

$$
R_{+, *}^{(2 m)}:=R_{+}^{(2 m)}-\left(\widehat{R_{+}^{y}}(0, \xi), y\right)-\left\langle\widehat{R_{+}^{z \bar{z}}}(0, \xi) z, \bar{z}\right\rangle .
$$

As a whole, the homological equation obeyed by $F$ reads:

$$
\Gamma\left(\left\{\tilde{H}_{0}, F\right\}+R_{*}^{(2 m)}+R_{+, *}^{(2 m)}\right)=0 .
$$

Let

$$
\begin{aligned}
& B^{(m+1)}:=\widetilde{B}^{(m+1)}+\widehat{R_{+}^{z \bar{z}}}(0, \xi)=B^{(m)}+\widehat{R^{z \bar{z}}}(0, \xi)+\widehat{R_{+}^{z \bar{z}}}(0, \xi), \\
& \omega^{(m+1)}:=\widetilde{\omega}^{(m+1)}+\widehat{R_{+}^{y}}(0, \xi)=\omega^{(m)}+\widehat{R^{y}}(0, \xi)+\widehat{R_{+}^{y}}(0, \xi) .
\end{aligned}
$$

In view of (5.27), (5.28) and (5.44), we get

$$
H^{(m+1)}=H_{0}^{(m+1)}+R^{(m+1)}+P^{(m+1)},
$$

where

$$
\begin{aligned}
H_{0}^{(m+1)}=( & \left.\omega^{(m+1)}, y\right)+\sum_{j \in \mathbb{Z}^{d}} \lambda_{j} z_{j} \bar{z}_{j}+\left\langle B^{(m+1)} z, \bar{z}\right\rangle, \\
R^{(m+1)}= & (1-\Gamma)\left(\left\{\tilde{H}_{0}^{(m+1)}, F\right\}+R_{*}^{(2 m)}+R_{+, *}^{(2 m)}\right) \\
& +\left\{R_{*}^{(2 m)}, F\right\} \\
& +\int_{0}^{1} \int_{0}^{t}\left\{\left\{H^{(m)}, F\right\}, F\right\} \circ X_{F}^{\tau} d \tau d t, \\
P^{(m+1)}=R^{(3 m)}+ & P^{(m)}+R_{+}^{(3 m)}=O\left(|y|^{2}+|y|\|z\|_{p}+\|z\|_{p}^{3}\right) .
\end{aligned}
$$

Recall

$$
\omega=\tilde{\omega}^{(m+1)} .
$$

Writing (5.44) explicitly, we have the following homological equations:

$$
\begin{gathered}
\omega \cdot \partial_{x} F^{x}=\Gamma R^{x} \\
\omega \cdot \partial_{x} F^{y}=\Gamma\left(R^{y}-\widehat{R^{y}}(0, \xi)+R_{+}^{y}-\widehat{R_{+}^{y}}(0, \xi)\right), \\
\Gamma\left(\omega \cdot \partial_{x} F^{z}+\mathbf{i}\left(\left(\Lambda+B^{T}\right) F^{z}\right)\right)=-\Gamma\left(R^{z}+R_{+}^{z}\right), \\
\Gamma\left(\omega \cdot \partial_{x} F^{\bar{z}}-\mathbf{i}\left((\Lambda+B) F^{\bar{z}}\right)\right)=-\Gamma\left(R^{\bar{z}}+R_{+}^{\bar{z}}\right),
\end{gathered}
$$




$$
\begin{gathered}
\Gamma\left(\omega \cdot \partial_{x} F^{z z}+\mathbf{i}\left(\left(\Lambda+B^{T}\right) F^{z z}+F^{z z}(\Lambda+B)\right)\right)=-\Gamma\left(R^{z z}+R_{+}^{z z}\right), \\
\Gamma\left(\omega \cdot \partial_{x} F^{\bar{z} \bar{z}}-\mathbf{i}\left((\Lambda+B) F^{\bar{z} \bar{z}}+F^{\bar{z} \bar{z}}\left(\Lambda+B^{T}\right)\right)\right)=\Gamma\left(R^{\bar{z} \bar{z}}+R_{+}^{\bar{z} \bar{z}}\right), \\
\Gamma\left(\omega \cdot \partial_{x} F^{z \bar{z}}-\mathbf{i}\left((\Lambda+B) F^{z \bar{z}}-F^{z \bar{z}}(\Lambda+B)\right)\right)=\Gamma\left(R^{z \bar{z}}+R_{+}^{z \bar{z}}\right)-\widehat{R^{z \bar{z}}}(0, \xi)-\widehat{R_{+}^{z \bar{z}}}(0, \xi),
\end{gathered}
$$

where $\Gamma F^{x}=F^{x}, \Gamma F^{y}=F^{y}, \Gamma F^{z}=F^{z}, \Gamma F^{\bar{z}}=F^{\bar{z}}, \Gamma F^{z z}=F^{z z}, \Gamma F^{\bar{z} \bar{z}}=F^{\bar{z} \bar{z}}, \Gamma F^{z z}=F^{z z}$ and $\widehat{F z \bar{z}}(0, \xi)=0$.

Finally, we point out that $B$ is self-adjoint in $\ell_{2}$. By Assumption $\mathbf{D},\langle B z, \bar{z}\rangle$ is real when $\bar{z}$ is regarded as the complex conjugate of $z$. Thus,

$$
\langle B z, \bar{z}\rangle=\overline{\langle B z, \bar{z}\rangle}=\langle\bar{B} \bar{z}, z\rangle=\left\langle\bar{z}, \bar{B}^{T} z\right\rangle=\left\langle\bar{B}^{T} z, \bar{z}\right\rangle,
$$

where the bar is the complex conjugate. It follows that $B$ is self-adjoint in $\ell_{2}\left(\mathbb{Z}^{d}\right)$, that is, $B^{T}=\bar{B}$.

\section{Solutions to the homological equations}

We will solve those homological equations (5.54)-(5.60) in the following order:

$$
(5.54) \Rightarrow(5.56) \text { and }(5.57) \Rightarrow(5.55) \Rightarrow(5.58) \text { and }(5.59) \text { and (5.60). }
$$

Recall

$$
\omega=\tilde{\omega}^{(m+1)}=\omega^{(m)}+\widehat{R}^{y}(0, \xi),
$$

by (5.27) and (5.13) and (5.11). In view of (5.5) and (4.5) and (4.6),

$$
\partial_{\xi} \omega=\partial_{\xi} \omega^{0}+O\left(\varepsilon_{0}\right) \text {. }
$$

Using Assumption A, the map $\omega: \mathscr{O}_{m} \rightarrow \omega\left(\mathscr{O}_{m}\right)$ is a diffeomorphism between $\mathscr{O}_{m}$ and its image $\omega\left(\mathscr{O}_{m}\right)$ and

$$
\left|\operatorname{det} \frac{\partial \omega}{\partial \xi}\right| \geq c_{1} / 2>0 \text {. }
$$

Therefore, we assume $\omega(\xi) \equiv \xi$ without loss of generality. In this section, we always let $K=K_{m}$.

Lemma 6.1. (Solutions to (5.54) $)$ There a subset $\mathscr{O}_{1 m} \subset \mathscr{O}_{m}$ with

$$
\text { Meas } \mathscr{O}_{1 m}=\left(\text { Meas } \mathscr{O}_{m}\right)\left(1-O\left(K^{-C}\right)\right) \text {, }
$$

such that for any $\xi \in \mathscr{O}_{1 m}$, Eq. (5.54) has a unique solution $F^{x}(x): \mathbb{T}_{s_{m}^{1}}^{N} \times \mathscr{O}_{1 m} \rightarrow \mathbb{C}^{N}$ which is analytic in $x \in \mathbb{T}_{s_{m}^{1}}^{N}$ and smooth in $\xi \in \mathscr{O}_{1 m}$ and obeys

$$
\left|\left\lfloor F^{x}\right\rceil\right|_{s_{m}^{1}, \mathscr{O}_{1 m}} \leq C(m) \varepsilon_{m},\left|\left\lfloor\partial_{\xi} F^{x}\right\rceil\right|_{s_{m}^{1}, \mathscr{O}_{1 m}} \leq C(m) \varepsilon_{m} .
$$

Proof. Note (5.4). The proof is finished by a standard argument in KAM theory. We omit it here.

Lemma 6.2. (Solutions to (5.56) and (5.57) Let $u=z$ or $u=\bar{z}$. There a subset $\mathscr{O}_{2 m} \subset \mathscr{O}_{m}$ with

$$
\text { Meas } \mathscr{O}_{2 m}=\left(\text { Meas } \mathscr{O}_{m}\right)\left(1-O\left(K^{-C}\right)\right)
$$

such that for any $\xi \in \mathscr{O}_{2 m}$, each equation of (5.56) and (5.57) has a unique solution $F^{u}(x)=\mathscr{F}_{1}^{u}(\xi)+\mathscr{F}_{2}^{u}(x, \xi)$, where $\mathscr{F}_{1}^{u}(\xi): \mathscr{O}_{2 m} \rightarrow h_{p}$ is smooth in $\xi \in \mathscr{O}_{2 m}$ and obeys

$$
\sup _{\xi \in \mathscr{O}_{2 m}}\left\|\left\lfloor\mathscr{F}_{1}^{u}(\xi)\right\rceil\right\|_{p} \leq K^{C} \varepsilon_{m}, \sup _{\xi \in \mathscr{O}_{2 m}}\left\|\left\lfloor\partial_{\xi} \mathscr{F}_{1}^{u}\right\rceil\right\|_{p} \leq K^{C} \varepsilon_{m}
$$

and where $\mathscr{F}_{2}^{u}: \mathbb{T}_{s_{m}^{2}}^{N} \times \mathscr{O}_{2 m} \rightarrow h_{q}$ which is analytic in $x \in \mathbb{T}_{s_{m}^{2}}^{N}$ and smooth in $\xi \in \mathscr{O}_{2 m}$ and obeys

$$
\left\|\left\lfloor\mathscr{F}_{2}^{u}\right\rceil\right\|_{q, s_{m}^{2}, \mathscr{O}_{2 m}} \leq K^{C} \varepsilon_{m}, \quad\left\|\left\lfloor\partial_{\xi} \mathscr{F}_{2}^{u}\right\rceil\right\|_{q, s_{m}^{2}, \mathscr{O}_{2 m}} \leq K^{C} \varepsilon_{m}
$$


Proof. We point out that for any vector function $f(x, \xi),\|f\|_{q, s, \mathscr{O}}=\|\lfloor f\rceil\|_{q, s, \mathscr{O}}$. Recall that $\tilde{P}^{(m)}=R^{(3 m)}+P^{(m)}$. By (5.8) and (4.12) with $l=m$,

$$
\left|\left\lfloor X_{\tilde{P}(m)}\right\rceil\right|_{q, D_{p}\left(s_{m}, r_{m}\right) \times \mathscr{O}_{m}} \leq C(m),\left|\left\lfloor\partial_{\xi} X_{\tilde{P}(m)}\right\rceil\right|_{q, D_{p}\left(s_{m}, r_{m}\right) \times \mathscr{O}_{m}} \leq C(m) .
$$

Applying (6.2) and (6.5) to (5.38) and (5.39), we get

$$
\left\|\left\lfloor R_{+}^{u}\right\rceil\right\|_{q, s_{m}^{1}, \mathscr{O}_{1 m}} \leq C(m) \varepsilon_{m},\left\|\left\lfloor\partial_{\xi} R_{+}^{u}\right\rceil\right\|_{q, s_{m}^{1}, \mathscr{O}_{1 m}} \leq C(m) \varepsilon_{m} .
$$

Set $\mathscr{R}^{u}=R^{u}+R_{+}^{u}$. By (6.6) and (5.6),

$$
\left\|\left\lfloor\mathscr{R}^{u}\right\rceil\right\|_{q, s_{m}^{1}, \mathscr{O}_{1 m}} \leq C(m) \varepsilon_{m},\left\|\left\lfloor\partial_{\xi} \mathscr{R}^{u}\right\rceil\right\|_{q, s_{m}^{1}, \mathscr{O}_{1 m}} \leq C(m) \varepsilon_{m} .
$$

Decompose $F^{u}=\mathscr{F}_{1}^{u}(\xi)+\mathscr{F}_{2}^{u}(x, \xi)$ with

$$
\mathscr{F}_{1}^{u}(\xi)=\widehat{F}^{u}(0, \xi), \quad \mathscr{F}_{2}^{u}(x, \xi)=\sum_{0<|k| \leq K} \widehat{F}^{u}(k) e^{\mathbf{i}\langle k, x\rangle},
$$

and decompose $\mathscr{R}^{u}=\mathscr{R}_{1}^{u}(\xi)+\mathscr{R}_{2}^{u}(x, \xi)$ in the same way as done in $F^{u}$. When $k=0$, by (5.56) and (5.57),

$$
\begin{aligned}
\widehat{F^{u}}(0, \xi) & =\mathbf{i}(\Lambda+B)^{-1}\left(\widehat{\mathscr{R}}^{u}(0, \xi)\right) \\
& =\mathbf{i}\left(1+\Lambda^{-1} B\right)^{-1}\left(\Lambda^{-1} \widehat{\mathscr{R}}^{u}(0, \xi)\right) .
\end{aligned}
$$

Note $\left\|\Lambda^{-1} B\right\|_{h_{p} \rightarrow h_{p}}=\|B\|_{h_{p} \rightarrow h_{q}} \leq \varepsilon_{0}$ and

$$
\begin{gathered}
\left\|\Lambda^{-1} \widehat{\mathscr{R}}^{u}(0, \xi)\right\|_{p} \leq\left\|\left\lfloor\mathscr{R}^{u}\right\rceil\right\|_{q, s_{m}^{1}, \mathscr{O}_{1 m}} \leq C(m) \varepsilon_{m}, \\
\left\|\Lambda^{-1} \partial_{\xi} \widehat{\mathscr{R}}^{u}(0, \xi)\right\|_{p} \leq\left\|\left\lfloor\partial_{\xi} \mathscr{R}^{u}\right\rceil\right\|_{q, s_{m}^{1}, \mathscr{O}_{1 m}} \leq C(m) \varepsilon_{m} .
\end{gathered}
$$

So

$$
\|\widehat{F u}(0, \xi)\|_{p} \leq\left\|\left(1+\Lambda^{-1} B\right)^{-1}\right\|_{h_{p} \rightarrow h_{p}}\left\|\Lambda^{-1} \widehat{R^{u}}(0, \xi)\right\|_{p} \leq C(m) \varepsilon_{m}, \quad\left\|\partial_{\xi} \widehat{F^{u}}(0, \xi)\right\|_{p} \leq C(m) \varepsilon_{m} .
$$

Considering the decompositions $F^{u}=\mathscr{F}_{1}^{u}+\mathscr{F}_{2}^{u}$ and $\mathscr{R}=\mathscr{R}_{1}^{u}+\mathscr{R}_{2}^{u}$, we see that $\mathscr{F}_{2}$ obeys

$$
\Gamma\left(\omega \cdot \partial_{x} \mathscr{F}_{2}^{u} \pm \mathbf{i}\left((\Lambda+B) \mathscr{F}_{2}^{u}\right)=\Gamma \mathscr{R}_{2}^{u},\right.
$$

where

$$
\mathscr{R}_{2}^{u}=R^{u}(x, \xi)+R_{+}^{u}(x, \xi)-\widehat{R^{u}}(0)-\widehat{R_{+}^{u}}(0), \int_{\mathbb{T}^{N}} \mathscr{R}_{2}^{u}(x, \xi) d x=0 .
$$

By Lemma2.1, one gets that there exists a subset $\mathscr{O}_{2 m} \subset \mathscr{O}_{m}$ with

$$
\text { measure } \mathscr{O}_{2 m}=\left(\text { measure } \mathscr{O}_{m}\right)\left(1-K_{m}^{-C}\right)
$$

such that

$$
\begin{aligned}
& \left\|\left\lfloor\mathscr{F}_{2}^{u}\right\rceil\right\|_{q, s_{m}^{2}, \mathscr{O}_{2 m}} \leq K^{C}\left\|\left\lfloor\mathscr{R}_{2}^{u}\right\rceil\right\|_{q, s_{m}, \mathscr{O}_{1 m}} \leq K^{C} \varepsilon_{m}, \\
& \left\|\left\lfloor\partial_{\xi} \mathscr{F}_{2}^{u}\right\rceil\right\|_{q, s_{m}^{2}, \mathscr{O}_{2 m}} \leq K^{C}\left(\left\|\left\lfloor\mathscr{R}_{2}^{u}\right\rceil\right\|_{q, s_{m}^{2}, \mathscr{O}_{1 m}}+\left\|\left\lfloor\partial_{\xi} \mathscr{R}_{2}^{u}\right\rceil\right\|_{q, s_{m}^{2}, \mathscr{O}_{1 m}}\right) \leq K^{C} \varepsilon_{m}
\end{aligned}
$$

This completes the proof of this lemma.

Remark 10. Although $\left\|\widehat{F^{u}}(0, \xi)\right\|_{p}$ is small, the norm $\left\|\widehat{F^{u}}(0, \xi)\right\|_{q}$ may be infinite, which is precarious. Fortunately the vector $\hat{F}^{u}(0, \xi)$ is independent of variables $(x, y, z, \bar{z})$, and $\left\|\widehat{F^{u}}(k, \xi)\right\|_{q}$ is small for $k \neq 0$. By (6.3) and 6.4], we have $\left\|\left\lfloor F^{u}\right\rceil\right\|_{p, s_{m}^{2}, \mathscr{O}_{2 m}} \leq K^{c} \varepsilon_{m},\left\|\left\lfloor\partial_{\xi} F^{u}\right\rceil\right\|_{p, s_{m}^{2}, \mathscr{O}_{2 m}} \leq K^{c} \varepsilon_{m}$. 
Lemma 6.3. (Solution to (5.55)) There a subset $\mathscr{O}_{3 m} \subset \mathscr{O}_{m}$ with

$$
\text { Meas } \mathscr{O}_{3 m}=\left(\operatorname{Meas} \mathscr{O}_{m}\right)\left(1-O\left(K^{-C}\right)\right)
$$

such that for any $\xi \in \mathscr{O}_{3 m}$, the equation (5.55) has a unique solution $F^{y}(x, \xi): \mathbb{T}_{s_{m}^{3}}^{N} \times \mathscr{O}_{3 m} \rightarrow \mathbb{C}^{N}$ is smooth in $\xi \in \mathscr{O}_{3 m}$ and obeys

$$
\left|F^{y}(x, \xi)\right|_{s_{m}^{3}, \mathscr{O}_{3 m}} \leq K^{C} \varepsilon_{m},\left|\partial_{\xi} F^{y}(x, \xi)\right|_{s_{m}^{3}, \mathscr{O}_{3 m}} \leq K^{C} \varepsilon_{m} .
$$

Proof. Applying (6.2), 6.3, 6.4, 6.5) to (5.37), one gets

$$
\begin{aligned}
& \left.\| R_{+}^{y}\right\rceil\left.\right|_{s_{m}^{2}, \mathscr{O}_{2 m}} \leq C\left(\left\|\left.\left\lfloor\partial_{x} F^{x}\right\rceil\right|_{s_{m}^{2}, \mathscr{O}_{2 m}}+\right\|\left\lfloor F^{z}\right\rceil\left\|_{p, s_{m}^{2}, \mathscr{O}_{2 m}}+\right\|\left\lfloor F^{\bar{z}}\right\rceil \|_{p, s_{m}^{2}, \mathscr{O}_{2 m}}\right) \leq K^{C} \varepsilon_{m}, \\
& \|\left.\left\lfloor\partial_{\xi} R_{+}^{y}\right\rceil\right|_{s_{m}^{2}, \mathscr{O}_{2 m}} \leq C\left(\sum_{t=0}^{1}\left|\left\lfloor\partial_{\xi}^{t} \partial_{x} F^{x}\right\rceil\right|_{s_{m}^{2}, \mathscr{O}_{2 m}}+\left\|\left\lfloor\partial_{\xi}^{t} F^{z}\right\rceil\right\|_{s_{m}^{2}, \mathscr{O}_{2 m}}+\|\left.\left\lfloor\partial_{\xi}^{t} F^{\bar{z}}\right\rceil\right|_{s_{m}^{2}, \mathscr{O}_{2 m}}\right) \leq K^{C} \varepsilon_{m} .
\end{aligned}
$$

By (6.12) and (5.5), the proof is completed by standard KAM procedure. We omit it.

Lemma 6.4. (Solutions to (5.58) and (5.59) and (5.60) Let $u, v \in\{z, \bar{z}\}$. There a subset $\mathscr{O}_{4 m} \subset \mathscr{O}_{m}$ with

$$
\text { Meas } \mathscr{O}_{4 m}=\left(\text { Meas } \mathscr{O}_{m}\right)\left(1-O\left(K^{-C}\right)\right)
$$

such that for any $\xi \in \mathscr{O}_{4 m}$, each of equations (5.58) and (5.59) and (5.60) has a unique solution

$$
\left\lfloor F^{u v}(x, \xi)\right\rceil: \mathbb{T}_{s_{m}^{4}}^{N} \times \mathscr{O}_{4 m} \rightarrow \mathscr{L}\left(h_{p}, h_{q}\right)
$$

is smooth in $\xi \in \mathscr{O}_{4 m}$ and analytic in $x \in \mathbb{T}_{s_{m}^{4}}^{N}$ and obeys

$$
\left\{\begin{array}{l}
\sup _{\mathbb{T}_{s_{m}}^{N} \times \mathscr{O}_{4 m}}\left\|\left\lfloor F^{u v}(x, \xi)-\widehat{F^{u v}}(0, \xi)\right\rceil\right\|_{h_{p} \rightarrow h_{q}} \leq K^{C} \varepsilon_{m}, \\
\sup _{\mathbb{T}_{s_{m}^{4}}^{N} \times \mathscr{O}_{4 m}}\left\|\left\lfloor\partial_{\xi}\left(F^{u v}(x, \xi)-\widehat{F^{u v}}(0, \xi)\right)\right\rceil\right\|_{h_{p} \rightarrow h_{q}} \leq K^{C} \varepsilon_{m}
\end{array}\right.
$$

and

$$
\left.\sup _{\mathscr{O}_{4 m}} \| \widehat{F^{u v}}(0, \xi)\right\rceil\left\|_{h_{\tilde{p}} \rightarrow h_{\tilde{p}}} \leq K^{C} \varepsilon_{m}, \sup _{\mathscr{O}_{4 m}}\right\|\left\lfloor\partial_{\xi} \widehat{F^{u v}}(0, \xi)\right\rceil \|_{h_{\tilde{p}} \rightarrow h_{\tilde{p}}} \leq K^{C} \varepsilon_{m}, \tilde{p} \in\{p, q\} .
$$

Proof. We firstly give the estimates of $R_{+}^{u v}$ with $u, v \in\{z, \bar{z}\}$ which are defined in (5.40), (5.41) and (5.42). Without loss of generality, we only give the estimate of $R_{+}^{z z}$ defined in (5.40). According to the decomposition in Lemma 6.2 write $F^{u}=\mathscr{F}_{1}^{u}(\xi)+\mathscr{F}_{2}^{u}(x, \xi)$ where $u \in\{z, \bar{z}\}$. A key observation is

$$
\partial_{x} \mathscr{F}_{1}^{u}(\xi) \equiv 0
$$

Thus

$$
\partial_{y} \tilde{P}^{(m)} \cdot \partial_{x}\left(\partial_{z}\left(\left\langle F^{z}, z\right\rangle+\left\langle F^{\bar{z}}, \bar{z}\right\rangle\right)\right)=\partial_{y} \tilde{P}^{(m)} \cdot \partial_{x} \mathscr{F}_{2}^{z}
$$

By 6.4 and 6.5),

$$
\partial_{y} \tilde{P}^{(m)} \cdot \partial_{x} \mathscr{F}_{2}^{z}: D_{p}\left(s_{m}^{2}, r_{m}^{2}\right) \times \mathscr{O}_{2 m} \rightarrow h_{q}
$$

with

$$
\sup _{D_{p}\left(s_{m}^{2}, r_{m}^{2}\right) \times \mathscr{O}_{2 m}}\left\|\left\lfloor\partial_{y} \tilde{P}^{(m)} \cdot \partial_{x} \mathscr{F}_{2}^{z}\right\rceil\right\|_{h_{q}} \leq C(m) \varepsilon_{m}, \sup _{D_{p}\left(s_{m}^{2}, r_{m}^{2}\right) \times \mathscr{O}_{2 m}}\left\|\left\lfloor\partial_{\xi}\left(\partial_{y} \tilde{P}^{(m)} \cdot \partial_{x} \mathscr{F}_{2}^{z}\right)\right\rceil\right\|_{h_{q}} \leq C(m) \varepsilon_{m} .
$$

Set

$$
A_{1}=\left.\partial_{z}\left(\partial_{y} \tilde{P}^{(m)} \cdot \partial_{x} \mathscr{F}_{2}^{z}\right)\right|_{y=0, z=\bar{z}=0}
$$


By the Cauchy estimate and using $(6.16$,

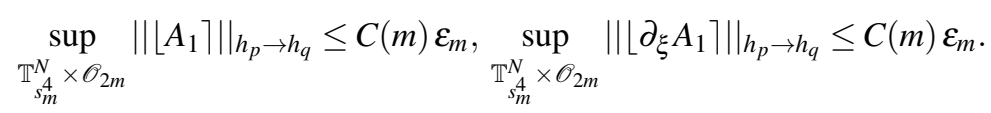

By (6.5) and using the Cauchy estimate, we have that $\partial_{z}^{2} \tilde{P}^{(m)}: h_{p} \rightarrow h_{q}$ with

$$
\sup _{D_{p}\left(s_{m}, r_{m}\right) \times \mathscr{O}_{m}}\left\|\left\lfloor\partial_{z}^{2} \tilde{P}^{(m)}\right\rceil\right\|_{h_{p} \rightarrow h_{q}} \leq C \frac{1}{r_{m}-r_{m}^{2}}\left\|\left\lfloor X_{\left.\tilde{P}^{(m)}\right)}\right]\right\|_{h_{p} \rightarrow h_{q}} \leq C(m) .
$$

Using (6.3) and (6.4),

$$
\left\|\left\lfloor F^{u}\right\rceil\right\|_{p, s_{m}^{2}, \mathscr{O}_{2 m}} \leq K^{C} \varepsilon_{m},\left\|\left\lfloor\partial_{\xi} F^{u}\right\rceil\right\|_{p, s_{m}^{2}, \mathscr{O}_{2 m}} \leq K^{C} \varepsilon_{m}, u \in\{z, \bar{z}\}
$$

where we have used $C(m) K^{C} \leq K^{C}$ by enlarging the last $C$. Combining the last two inequalities, we have

$$
\left\|\left\lfloor\left(\partial_{z}^{2} \tilde{P}^{(m)}\right) F^{u}\right\rceil\right\|_{q, s_{m}^{2}, \mathscr{O}_{2 m}} \leq K^{C} \varepsilon_{m},\left\|\left\lfloor\partial_{\xi}\left(\partial_{z}^{2} \tilde{P}^{(m)}\right) F^{u}\right\rceil\right\|_{q, s_{m}^{2}, \mathscr{O}_{2 m}} \leq K^{C} \varepsilon_{m} .
$$

Let

$$
A_{2}:=-\left.\partial_{z} \partial_{z}\left(\left\langle\partial_{z} \tilde{P}^{(m)}, F^{\bar{z}}\right\rangle+\left\langle\partial_{\bar{z}} \tilde{P}^{(m)}, F^{z}\right\rangle\right)\right|_{y=0, z=\bar{z}=0} .
$$

By Cauchy inequality and using 6.20 ,

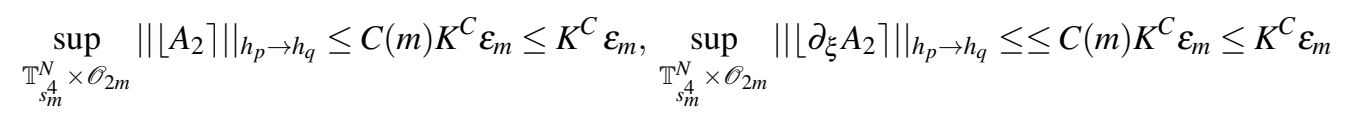

where the last constant $C$ in each inequality is enlarged. By (5.40), we have $R_{+}^{z z}=A_{1}+A_{2}$. It follows from 6.21 and (6.17) that

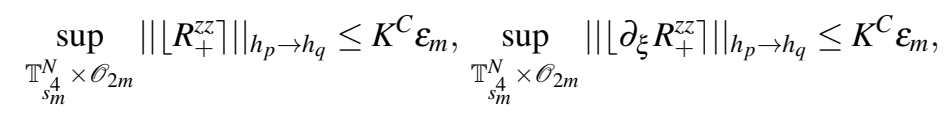

where $C$ is enlarged again. Similarly, the last inequality holds true for $R^{z \bar{z}}$ and $R^{\bar{z} \bar{z}}$, too. Noting (5.7), (6.22) and using Lemma 3.1, we finish the proof of this lemma.

\section{Estimates for new perturbation}

Note $D_{p}\left(s_{m}^{i}, r_{m}^{i}\right) \subset D_{p}\left(s_{m+1}, r_{m+1}\right)$ for $i=1,2,3,4$. Let $\mathscr{O}_{m+1}=\mathscr{O} \cap \mathscr{O}_{1 m} \cap \mathscr{O}_{2 m} \cap \mathscr{O}_{3 m} \cap \mathscr{O}_{4 m}$. By Lemmas 6.1, $6.2,6.3$ and 6.4 ,

$$
\text { Meas } \mathscr{O}_{m+1}=\left(\text { Meas } \mathscr{O}_{m}\right)\left(1-O\left(K_{m}^{-C}\right)\right) \text {. }
$$

Recall that $F$ is defined in (5.22), (5.23) and (5.24). The Hamiltonian vector field $X_{F}$ reads

$$
\left(\partial_{y} F(w),-\partial_{x} F(w), \mathbf{i} \partial_{\bar{z}} F(w),-\mathbf{i} \partial_{z} F(w)\right),
$$

where $(w, \xi)=(x, y, z, \bar{z} ; \xi) \in D_{p}\left(s_{m+1}, r_{m+1}\right) \times O_{m+1}$. Note that the tangent space $T_{w} \mathscr{P}^{p}=\mathscr{P}^{p}$ and $T_{w} \mathscr{P}^{q}=\mathscr{P}^{q}$. Denote by $\mathscr{V} \tilde{p}$ all functions which map

$$
D_{p}\left(s_{m+1}, r_{m+1}\right) \times O_{m+1} \rightarrow T_{w} \mathscr{P} \tilde{p}, \quad \tilde{p} \in\{p, q\} .
$$

Recall (1.14),

$$
\left|X_{F}\right|_{q, D_{p}(s, r) \times \mathscr{O}}=\sqrt{\left|\partial_{y} X_{F}\right|_{p, s, r, \mathscr{O}}^{2}+\left|\partial_{x} X_{F}\right|_{p, s, r, \mathscr{O}}^{2}+\left\|\left.\partial_{\bar{z}} X_{F}\right|_{p, q, s, r, \mathscr{O}} ^{2}+\right\| \partial_{z} X_{F} \|_{p, q, s, r, \mathscr{O}}^{2}},
$$

where $s=s_{m+1}, r=r_{m+1}$ and $\mathscr{O}=\mathscr{O}_{m+1}$. Since $s_{m+1}, r_{m+1}, \mathscr{O}_{m+1}$ and the domain $D_{p}\left(s_{m+1}, r_{m+1}\right)$ are fixed in this section, we write $|\cdot|_{q, D_{p}(s, r) \times \mathscr{O}}$ as $|\cdot|_{q}$. Similarly, we write $|\cdot|_{p}:=|\cdot|_{p, D_{p}(s, r) \times \mathscr{O}}$. We will denote by $\|\cdot\|_{\tilde{p}, \tilde{q}}$ the operator norm from $\mathscr{V} \tilde{p}$ to $\mathscr{V} \tilde{q}$ where $\tilde{p}, \tilde{q} \in\{p, q\}$. 
Lemma 7.1. For $(w, \xi)=(x, y, z, \bar{z} ; \xi) \in D_{p}\left(s_{m+1}, r_{m+1}\right) \times \mathscr{O}_{m+1}$, we have

$$
\left\|\left\lfloor\mathscr{D} X_{F(w, \xi)}\right)\right\|_{q, q} \leq(N+1)\left\|\mathscr{D}\left[X_{F(w(\theta(t)), \xi)}\right)\right\|_{q, q}
$$

and

$$
\left\|\left\lfloor\mathscr{D} X_{F(w, \xi)}\right]\right\|_{p, p} \leq(N+1)\left\|\mathscr{D}\left[X_{F(w(\theta(t)), \xi)}\right]\right\|_{p, p}
$$

Proof. Note

$$
\mathscr{D} X_{F(w, \xi)}=\left(\partial_{x} X_{F}, \partial_{y} X_{F}, \partial_{z} X_{F}, \partial_{\bar{z}} X_{F}\right)=\left(\begin{array}{cccc}
\partial_{x} \partial_{y} F & \partial_{y} \partial_{y} F & \partial_{z} \partial_{y} F & -\partial_{\bar{z}} \partial_{y} F \\
-\partial_{x} \partial_{x} F & -\partial_{y} \partial_{x} F & -\partial_{z} \partial_{x} F & \partial_{\bar{z}} \partial_{x} F \\
\mathbf{i} \partial_{x} \partial_{\bar{z}} F & \mathbf{i} \partial_{y} \partial_{\bar{z}} F & \mathbf{i} \partial_{z} \partial_{\bar{z}} F & \mathbf{i} \partial_{\bar{z}} \partial_{\bar{z}} F \\
-\mathbf{i} \partial_{x} \partial_{z} F & -\mathbf{i} \partial_{y} \partial_{z} F & -\mathbf{i} \partial_{z} \partial_{z} F & -\mathbf{i} \partial_{z} \partial_{\bar{z}} F
\end{array}\right),
$$

and

$$
\begin{aligned}
\mathscr{D}\left\lfloor X_{F(w, \xi)}\right) & \left(\partial_{x}\left\lfloor X_{F}\right\rceil, \partial_{y}\left\lfloor X_{F}\right\rceil, \partial_{z}\left\lfloor X_{F}\right\rceil, \partial_{\bar{z}}\left\lfloor X_{F}\right\rceil\right) \\
& =\left(\begin{array}{cccc}
\partial_{x}\left\lfloor\partial_{y} F\right\rceil & \partial_{y}\left\lfloor\partial_{y} F\right\rceil & \partial_{z}\left\lfloor\partial_{y} F\right\rceil & \partial_{\bar{z}}\left\lfloor\partial_{y} F\right\rceil \\
\partial_{x}\left\lfloor\partial_{x} F\right\rceil & \partial_{y}\left\lfloor\partial_{x} F\right\rceil & \partial_{z}\left\lfloor\partial_{x} F\right\rceil & \partial_{\bar{z}}\left\lfloor\partial_{x} F\right\rceil \\
\mathbf{i} \partial_{x}\left\lfloor\partial_{z} F\right\rceil & \mathbf{i} \partial_{y}\left\lfloor\partial_{z} F\right\rceil & \mathbf{i} \partial_{z}\left\lfloor\partial_{z} F\right\rceil & \mathbf{i} \partial_{\bar{z}}\left\lfloor\partial_{\bar{z}} F\right\rceil \\
-\mathbf{i} \partial_{x}\left\lfloor\partial_{z} F\right\rceil & -\mathbf{i} \partial_{y}\left\lfloor\partial_{z} F\right\rceil & -\mathbf{i} \partial_{z}\left\lfloor\partial_{z} F\right\rceil & -\mathbf{i} \partial_{z}\left\lfloor\partial_{z} F\right\rceil
\end{array}\right) \\
& =\left(\begin{array}{cccc}
1 & 0 & 0 & 0 \\
0 & 1 & 0 & 0 \\
0 & & \mathbf{i} & 0 \\
0 & 0 & 0 & -\mathbf{i}
\end{array}\right)\left(\begin{array}{cccc}
\partial_{x}\left\lfloor\partial_{y} F\right\rceil & \partial_{y}\left\lfloor\partial_{y} F\right\rceil & \partial_{z}\left\lfloor\partial_{y} F\right\rceil & \partial_{\bar{z}}\left\lfloor\partial_{y} F\right\rceil \\
\partial_{x}\left\lfloor\partial_{x} F\right\rceil & \partial_{y}\left\lfloor\partial_{x} F\right\rceil & \partial_{z}\left\lfloor\partial_{x} F\right\rceil & \partial_{\bar{z}}\left\lfloor\partial_{x} F\right\rceil \\
\partial_{x}\left\lfloor\partial_{z} F\right\rceil & \partial_{y}\left\lfloor\partial_{\bar{z}} F\right\rceil & \partial_{z}\left\lfloor\partial_{\bar{z}} F\right\rceil & \partial_{\bar{z}}\left\lfloor\partial_{\bar{z}} F\right\rceil \\
\partial_{x}\left\lfloor\partial_{z} F\right\rceil & \partial_{y}\left\lfloor\partial_{z} F\right\rceil & \partial_{z}\left\lfloor\partial_{z} F\right\rceil & \partial_{z}\left\lfloor\partial_{\bar{z}} F\right\rceil
\end{array}\right) \\
& :=\Xi_{0} \Theta .
\end{aligned}
$$

Note that $\Xi_{0}$ is a unitary operator from $\mathscr{V}^{q}$ to $\mathscr{V}^{q}$. Thus

$$
\left\|\mathscr{D}\left[X_{F(w, \xi)}\right)\right\|_{q, q}=\|\Theta\|_{q, q} .
$$

If we write

$$
X_{F}=\sum_{k, \alpha, \beta, \gamma} C_{k \alpha \beta \gamma} e^{\mathbf{i}(k, x)} y^{\gamma} z^{\alpha} \bar{z}^{\beta},
$$

we find that the indices $\alpha, \beta, \gamma$ of $z, \bar{z}, y$ are non-negative integer vector, except for the index $k$ of $x$. So we have

$$
\left\lfloor\mathscr{D} X_{F(w, \xi)}\right)=\left(\begin{array}{llll}
\left\lfloor\partial_{x}\left\lfloor\partial_{y} F\right\rceil\right\rceil & \partial_{y}\left\lfloor\partial_{y} F\right\rceil & \partial_{z}\left\lfloor\partial_{y} F\right\rceil & \partial_{\bar{z}}\left\lfloor\partial_{y} F\right\rceil \\
\left\lfloor\partial_{x}\left\lfloor\partial_{x} F\right\rceil\right\rceil & \partial_{y}\left\lfloor\partial_{x} F\right\rceil & \partial_{z}\left\lfloor\partial_{x} F\right\rceil & \left.\partial_{\bar{z}} \partial_{x} F\right\rceil \\
\left\lfloor\partial_{x}\left\lfloor\partial_{\bar{z}} F\right\rceil\right\rceil & \partial_{y}\left\lfloor\partial_{\bar{z}} F\right\rceil & \partial_{z}\left\lfloor\partial_{z} F\right\rceil & \partial_{\bar{z}}\left\lfloor\partial_{z} F\right\rceil \\
\left\lfloor\partial_{x}\left\lfloor\partial_{z} F\right\rceil\right\rceil & \partial_{y}\left\lfloor\partial_{z} F\right\rceil & \partial_{z}\left\lfloor\partial_{z} F\right\rceil & \partial_{\bar{z}}\left\lfloor\partial_{z} F\right\rceil
\end{array}\right)
$$

We see that all entries of $\mathscr{D}\left[X_{F(w, \xi)}\right]$ and $\left\lfloor\mathscr{D} X_{F(w, \xi)}\right]$ are the same except those entries in the first columns of them. Thus we partition $\left\lfloor\mathscr{D} X_{F(w, \xi)}\right\rceil:=\digamma_{1}+\digamma_{2}$, where

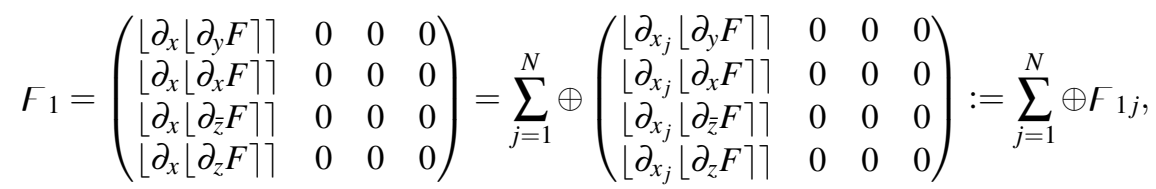

and

$$
\digamma_{2}=\left(\begin{array}{llll}
0 & \partial_{y}\left\lfloor\partial_{y} F\right\rceil & \partial_{z}\left\lfloor\partial_{y} F\right\rceil & \partial_{z}\left\lfloor\partial_{y} F\right\rceil \\
0 & \partial_{y}\left\lfloor\partial_{x} F\right\rceil & \partial_{z}\left\lfloor\partial_{x} F\right\rceil & \partial_{z}\left\lfloor\partial_{x} F\right\rceil \\
0 & \partial_{y}\left\lfloor\partial_{\bar{z}} F\right\rceil & \partial_{z}\left\lfloor\partial_{z} F\right\rceil & \partial_{z}\left\lfloor\partial_{z} F\right\rceil \\
0 & \partial_{y}\left\lfloor\partial_{z} F\right\rceil & \partial_{z}\left\lfloor\partial_{z} F\right\rceil & \partial_{\bar{z}}\left\lfloor\partial_{z} F\right\rceil
\end{array}\right)
$$


We see that

$$
\Theta=\tilde{\digamma}_{1}+\digamma_{2},
$$

where

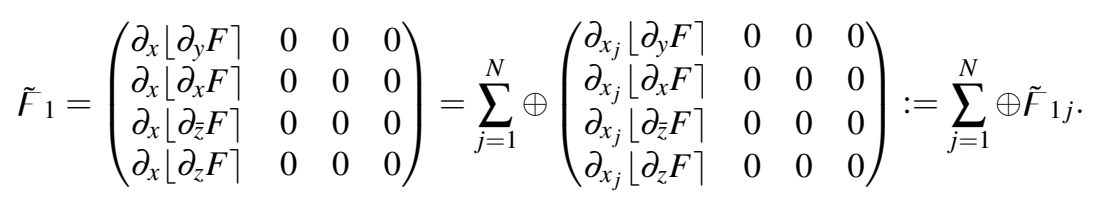

By Lemma12.1, we have

$$
\left\|\digamma_{2}\right\|_{q, q} \leq\|\Theta\|_{q, q}
$$

In order to compute $\left\|\digamma_{1}\right\|_{q, q}$, we write

$$
\partial_{u} F=\sum_{k} e^{\mathbf{i}(k, x)} \sum_{\alpha \beta, \gamma} C_{k, \alpha, \beta, \gamma ; u} y^{\gamma} z^{\alpha} \bar{z}^{\beta}, \quad u \in\{x, y, z, \bar{z}\} .
$$

Then

$$
\partial_{x_{j}}\left\lfloor\partial_{u} F\right\rceil=\sum_{k}\left(\mathbf{i} k_{j}\right) e^{\mathbf{i}(k, x)} \sum_{\alpha \beta, \gamma}\left|C_{k, \alpha, \beta, \gamma ; u}\right| y^{\gamma} z^{\alpha} \bar{z}^{\beta}:=\sum_{k}\left(\mathbf{i} k_{j}\right) e^{\mathbf{i}(k, x)} \sum_{\alpha \beta, \gamma} g_{k u}, \quad u \in\{x, y, z, \bar{z}\}
$$

and

$$
\left\lfloor\partial_{x_{j}}\left\lfloor\partial_{u} F\right\rceil\right\rceil=\sum_{k}\left|k_{j}\right| e^{\mathbf{i}(k, x)} \sum_{\alpha \beta, \gamma}\left|C_{k, \alpha, \beta, \gamma ; u}\right| y^{\gamma} z^{\alpha} \bar{z}^{\beta}=\sum_{k}|k| e^{\mathbf{i}(k, x)} g_{k u}
$$

It follows that

$$
\tilde{\digamma}_{1 j}=\sum_{k}\left(\mathbf{i} k_{j}\right) e^{\mathbf{i}(k, x)}\left(\begin{array}{cccc}
g_{k y} & 0 & 0 & 0 \\
g_{k x} & 0 & 0 & 0 \\
g_{k \bar{z}} & 0 & 0 & 0 \\
g_{k z} & 0 & 0 & 0
\end{array}\right), \digamma_{1 j}=\sum_{k}\left|k_{j}\right| e^{\mathbf{i}(k, x)}\left(\begin{array}{cccc}
g_{k y} & 0 & 0 & 0 \\
g_{k x} & 0 & 0 & 0 \\
g_{k \bar{z}} & 0 & 0 & 0 \\
g_{k z} & 0 & 0 & 0
\end{array}\right) .
$$

Let $g_{1 j}=\left(g_{1 j}^{y}, g_{1 j}^{x} g_{1 j}^{\bar{z}}, g_{1 j}^{z}\right)^{T}\left(\tilde{g}_{1 j}\right.$, respectively) be the first column of the operator $\digamma_{1 j}\left(\tilde{\digamma}_{1 j}\right.$, respectively). Note that all columns of $\digamma_{1 j}\left(\tilde{\digamma}_{1 j}\right.$, respectively) are zero vectors except $g_{1 j}\left(\tilde{g}_{1 j}\right.$, respectively). Thus

$$
\left\|\tilde{\digamma}_{1 j}\right\|_{q, q}=\left|\tilde{g}_{1 j}\right|_{q},\left\|\digamma_{1 j}\right\|_{q, q}=\left|g_{1 j}\right|_{q} .
$$

By the definition of $|\cdot|_{q}$,

$$
\begin{aligned}
\left|g_{1 g}\right|_{q}^{2} & =\left|g_{1 j}^{y}\right|_{p, s, r, \mathscr{O}}^{2}+\left|g_{1 j}^{x}\right|_{p, s, r, \mathscr{O}}^{2}+\left\|g_{1 j}^{\bar{z}}\right\|_{p, q, s, r, \mathscr{O}}^{2}+|| g_{1 j}^{z}||_{p, q, s, r, \mathscr{O}}^{2} \\
& =\sup _{\xi \in \mathscr{O},|y| \leq r^{2},\|z\|_{p} \leq r,\|z\|_{p} \leq r} \sum_{k}\left|k_{j}\right|^{2}\left(\left|g_{k y}\right|^{2}+\left|g_{k x}\right|^{2}+\left.|| g_{k \bar{z}}\left\|_{q}^{2}+\right\| g_{k z}\right|_{q} ^{2}\right) \\
& =\left|\tilde{g}_{1 g}\right|_{q}^{2} .
\end{aligned}
$$

It follows

$$
\left\|\digamma_{1 j}\right\|_{q, q}=\left\|\tilde{\digamma}_{1 j}\right\|_{q, q} .
$$

Note that $\tilde{\digamma}_{1 j}$ is a column of $\Theta$. By Lemma 12.1 we have

$$
\left\|\digamma_{1 j}\right\|_{q, q}=\left\|\tilde{\digamma}_{1 j}\right\|_{q, q} \leq\|\Theta\|_{q, q} .
$$

Consequently,

$$
\left\|\left\lfloor\mathscr{D} X_{F(w, \xi)}\right\rceil\right\|_{q, q} \leq(N+1)\left\|\mathscr{D}\left[X_{F(w, \xi)}\right\rceil\right\|_{q, q} .
$$

In the proof above, by change $q$ by $p$ we have

$$
\left\|\left\lfloor\mathscr{D} X_{F(w, \xi)}\right]\right\|_{p, p} \leq(N+1)\left\|\mathscr{D}\left[X_{F(w, \xi)}\right]\right\|_{p, p} .
$$

This completes the proof. 
Lemma 7.2. $\operatorname{For}(w, \xi)=(x, y, z, \bar{z} ; \xi) \in D_{p}\left(s_{m+1}, r_{m+1}\right) \times \mathscr{O}_{m+1}$,

$$
\left\|\left\lfloor\mathscr{D} X_{F(w, \xi)}\right]\right\|_{p, p} \leq K_{m}^{C} \varepsilon_{m},\left\|\left\lfloor\mathscr{D} X_{F(w, \xi)}\right\rceil\right\|_{q, q} \leq K_{m}^{C} \varepsilon_{m}
$$

and

$$
\left\|\left\lfloor\partial_{\xi} \mathscr{D} X_{F(w, \xi)}\right]\right\|_{p, p} \leq K_{m}^{C} \varepsilon_{m},\left\|\left\lfloor\partial_{\xi} \mathscr{D} X_{F(w, \xi)}\right\rceil\right\|_{q, q} \leq K_{m}^{C} \varepsilon_{m}
$$

Proof. Let

$$
\begin{gathered}
f^{u}=\left\langle F^{z}(x, \xi), z\right\rangle+\left\langle F^{\bar{z}}(x, \xi), \bar{z}\right\rangle, \\
f_{0}^{u}:=\left\langle\widehat{F^{z}}(0), z\right\rangle+\left\langle\widehat{F^{\bar{z}}}(0), \bar{z}\right\rangle
\end{gathered}
$$

and

$$
\tilde{f}^{u}:=\left\langle F^{z}(x, \xi), z\right\rangle+\left\langle F^{\bar{z}}(x, \xi), \bar{z}\right\rangle-f_{0}^{u}=f^{u}-f_{0}^{u} .
$$

By Lemma 6.2 (i.e., (6.4)),

$$
\left\|\left\lfloor X_{\tilde{f}^{u}}\right\rceil\right\|_{p, q, s_{m}^{2}, r_{m}^{2}, \mathscr{O}_{2 m}} \leq K_{m}^{C} \varepsilon_{m} .
$$

Note $s_{m}^{4}-s_{m+1}>\frac{1}{\mathrm{Cm}^{2}}, r_{m}^{4}-r_{m+1}>\frac{1}{\mathrm{Cm}^{2}}$. By the Cauchy's estimate,

$$
\sup _{D_{p}\left(s_{m+1}, r_{m+1}\right) \times \mathscr{O}_{m+1}}\left\|\mathscr{D}\left[X_{\tilde{f}^{u}}\right]\right\|_{p, q} \leq K_{m}^{C} \varepsilon_{m} .
$$

Another important observation is

$$
\mathscr{D} X_{f_{0}^{u}} \equiv 0
$$

So

$$
\sup _{D_{p}\left(s_{m+1}, r_{m+1}\right) \times \mathscr{O}_{m+1}}\left\|\mathscr{D}\left\lfloor X_{f^{u}}\right\rceil\right\|_{p, q} \leq K_{m}^{C} \varepsilon_{m} .
$$

Note $h_{q} \subset h_{p}$. By (7.6), we have

$$
\sup _{D_{p}\left(s_{m+1}, r_{m+1}\right) \times \mathscr{O}_{m+1}}\left\|\mathscr{D}\left\lfloor X_{f^{u}}\right\rceil\right\|_{p, p} \leq K_{m}^{C} \varepsilon_{m}, \quad \sup _{D_{p}\left(s_{m+1}, r_{m+1}\right) \times \mathscr{O}_{m+1}}\left\|\mathscr{D}\left\lfloor X_{f^{u}}\right\rceil\right\|_{q, q} \leq K_{m}^{C} \varepsilon_{m} .
$$

Let

$$
f^{u u}:=\left\langle\left(F^{z z}(x, \xi)-\widehat{F^{z z}}(0, \xi)\right) z, z\right\rangle+\left\langle F^{z \bar{z}}(x, \xi) z, \bar{z}\right\rangle+\left\langle\left(F^{\bar{z} \bar{z}}(x, \xi)-\widehat{F^{z \bar{z}}}(0, \xi)\right) \bar{z}, \bar{z}\right\rangle .
$$

Note $\widehat{F z \bar{z}}(0, \xi)=0$. By (6.13) in Lemma 6.4 and Cauchy's inequality,

$$
\sup _{D_{p}\left(s_{m+1}, r_{m+1}\right) \times \mathscr{O}_{m+1}}\left\|\mathscr{D}\left\lfloor X_{f u u}\right\rceil\right\|_{p, q} \leq K_{m}^{C} \varepsilon_{m} .
$$

Thus

$$
\sup _{D_{p}\left(s_{m+1}, r_{m+1}\right) \times \mathscr{O}_{m+1}}\left\|\mathscr{D}\left\lfloor X_{f u u}\right\rceil\right\|_{p, p} \leq K_{m}^{C} \varepsilon_{m}, \quad \sup _{D_{p}\left(s_{m+1}, r_{m+1}\right) \times \mathscr{O}_{m+1}}\left\|\mathscr{D}\left[X_{f u u}\right\rceil\right\|_{q, q} \leq K_{m}^{C} \varepsilon_{m}
$$

Let

$$
f^{u u}(0):=\langle\widehat{F z z}(0, \xi) z, z\rangle+\langle\widehat{F \bar{z}}(0, \xi) \bar{z}, \bar{z}\rangle .
$$

Then

$$
\mathscr{D} X_{f^{u u}(0)}=\left(\begin{array}{cccc}
0 & 0 & 0 & 0 \\
0 & 0 & 0 & 0 \\
0 & 0 & 0 & \mathbf{i} \widehat{F^{z z z}}(0, \xi) \\
0 & 0 & -\mathbf{i} \widehat{F^{z z}}(0, \xi) & 0
\end{array}\right)
$$


which is independent of $(x, y, z, \bar{z})$. By (6.14) in Lemma 6.4 we have

$$
\sup _{D_{p}\left(s_{m+1}, r_{m+1}\right) \times \mathscr{O}_{m+1}}\left\|\mathscr{D}\left\lfloor X_{f^{u u}(0)}\right]\right\|_{p, p} \leq K_{m}^{C} \varepsilon_{m}, \quad \sup _{D_{p}\left(s_{m+1}, r_{m+1}\right) \times \mathscr{O}_{m+1}}\left\|\mathscr{D}\left\lfloor X_{f u u}(0)\right]\right\|_{q, q} \leq K_{m}^{C} \varepsilon_{m} .
$$

Let

$$
f^{x, y}:=F^{x}(x, \xi)+\left(F^{y}(x, \xi), y\right) .
$$

By Lemmas 6.1, 6.3 and Cauchy's inequality,

$$
\sup _{D_{p}\left(s_{m+1}, r_{m+1}\right) \times \mathscr{O}_{m+1}}\left\|\mathscr{D}\left[X_{f x, y}\right\rceil\right\|_{p, p} \leq K_{m}^{C} \varepsilon_{m}, \quad \sup _{D_{p}\left(s_{m+1}, r_{m+1}\right) \times \mathscr{O}_{m+1}}\left\|\mathscr{D}\left[X_{f} x, y\right\rceil\right\|_{q, q} \leq K_{m}^{C} \varepsilon_{m} .
$$

Consequently,

$$
\sup _{D_{p}\left(s_{m+1}, r_{m+1}\right) \times \mathscr{O}_{m+1}}\left\|\mathscr{D}\left[X_{F}\right\rceil\right\|_{p, p} \leq K_{m}^{C} \varepsilon_{m}, \quad \sup _{D_{p}\left(s_{m+1}, r_{m+1}\right) \times \mathscr{O}_{m+1}}\left\|\mathscr{D}\left[X_{F}\right\rceil\right\|_{q, q} \leq K_{m}^{C} \varepsilon_{m} .
$$

We proves (7.2) by using Lemma 7.1 By applying the method as above to $\partial_{\xi} X_{F}$, we can finish the proof of (7.3). We omit the detail. This completes the proof of this lemma.

Let $X_{F(w(t))}^{t}=w(t)$ be the flow of the Hamiltonian vector field $X_{F}$. Then

$$
w(t)-w(0)=\int_{0}^{t} X_{F(w(s), \xi)}^{s} d s .
$$

Thus, for $t \in[0,1]$,

$$
\begin{aligned}
|w(t)-w(0)|_{p} & \leq \int_{0}^{t}\left|X_{F(w(s), \xi)}-X_{F(w(0), \xi)}\right|_{p}+\int_{0}^{t}\left|X_{F(w(0), \xi)}\right|_{p} d s \\
& \leq K_{m} \varepsilon_{m}+\int_{0}^{t}|| \mathscr{D} X_{F(w(\theta(s)), \xi)}||_{p, p}|w(s)-w(0)|_{p} d s
\end{aligned}
$$

where $\theta(s) \in[0,1]$ is a function of $s$, and $\mathscr{D}$ is the tangent map of $X_{F}$.

By Lemma 7.2 and Gronwall's inequality,

$$
|w(t)-w(0)|_{p} \leq K_{m}^{C} \varepsilon_{m} \exp \left(\int_{0}^{1} \| \mathscr{D} X_{F(w(\theta(s)), \xi)}||_{p, p} d s\right) \leq K_{m}^{C} \varepsilon_{m},
$$

where $(w(0), \xi) \in D_{p}\left(s_{m+1}, r_{m+1}\right) \times \mathscr{O}_{m+1}$ and we have used that $\left\|\mathscr{D} X_{F(w(\theta(s)), \xi)}\right\|_{p, p} \leq\left\|\left\lfloor\mathscr{D} X_{F(w(\theta(s)), \xi)}\right\rceil\right\|_{p, p}$.

Note that $r_{m+1}+K_{m}^{C} \varepsilon_{m}<r_{m}, s_{m+1}+K_{m}^{C} \varepsilon_{m}<s_{m}$. The inequality (7.13) implies that there does exist the solution $w(t)$ for $t \in[0,1]$ and that $\Psi_{m}:=w(1)$ obeys

$$
\left|\Psi_{m}(w, \xi)-w\right|_{p} \leq K_{m}^{C} \varepsilon_{m}, \quad(w, \xi) \in D_{p}\left(s_{m+1}, r_{m+1}\right) \times \mathscr{O}_{m+1}
$$

and

$$
\Psi_{m}: D_{p}\left(s_{m+1}, r_{m+1}\right) \times \mathscr{O}_{m+1} \rightarrow D_{p}\left(s_{m}, r_{m}\right) \times \mathscr{O}_{m} .
$$

This actually proves 4.14 .

We are now in position to estimate (5.50). Recall that $R_{*}^{(2 m)}$ is defined in (5.18), (5.19) and (5.20). By Lemma5.1

$$
\left.\left\|\left.\left\lfloor X_{R_{*}^{(2 m)}}\right\rceil\right|_{q, D_{p}\left(s_{m}, r_{m}\right)} \leq C(m) \varepsilon_{m},\right\| \partial_{\xi} X_{R_{*}^{(2 m)}}\right|_{q, D_{p}\left(s_{m}, r_{m}\right)} \leq C(m) \varepsilon_{m} .
$$

Combining Lemma 7.2 and (7.14), we have

$$
\begin{aligned}
\left.\| X_{\left\{R_{*}^{(2 m)}, F\right\}}\right\rceil\left.\right|_{q, D_{p}\left(s_{m+1}, r_{m+1}\right)} & \leq\left\|\left\lfloor\mathscr{D} X_{R_{*}^{(2 m)}}\right\rceil\right\|\left\|_{p, q}\left|X_{F}\right|_{p}+\right\|\left\lfloor\mathscr{D} X_{F}\right\rceil\left\|\left.\right|_{q, q} \mid\left\lfloor X_{R_{*}^{(2 m)}}\right\rceil\right\|_{q} \\
& \leq 2 \varepsilon_{m} K_{m}^{C} \varepsilon_{m} \leq \varepsilon_{m+1}
\end{aligned}
$$


and

$$
\begin{aligned}
& \|\left.\partial_{\xi} X_{\left\{R_{*}^{(2 m)}, F\right\}}\right|_{q, D_{p}\left(s_{m+1}, r_{m+1}\right)} \\
\leq & \sup \left(\left\|\left\lfloor\partial_{\xi} \mathscr{D} X_{R_{*}^{(2 m)}}\right\rceil\right\|_{p, q}\right)\left|\left\lfloor X_{F}\right\rceil\left\|_{p}+\sup \left(\left\|\left\lfloor\mathscr{D} X_{R_{*}^{(2 m)}}\right)\right\|_{p, q}\right) \mid\left\lfloor X_{F}\right\rceil\right\|_{p}\right. \\
& +\sup \left(\left\|\left\lfloor\partial_{\xi} \mathscr{D} X_{F}\right\rceil\right\|_{q, q}\right) \mid\left\lfloor\left. X_{R_{*}^{(2 m)}}\right|_{q}+\sup \left(\left\|\left\lfloor\mathscr{D} X_{F}\right\rceil\right\|_{q, q}\right) \mid\left\lfloor\partial_{\xi} X_{R_{*}^{(2 m)}}^{(2 m} \|_{q}\right.\right. \\
\leq & 4 \varepsilon_{m} K_{m}^{C} \varepsilon_{m} \leq \varepsilon_{m+1},
\end{aligned}
$$

where the "sup" runs over $D_{p}\left(s_{m+1}, r_{m+1}\right) \times \mathscr{O}_{m+1}$.

Recalling $\lambda_{j} \approx|j|^{-\kappa}$ and Assumption $\mathbf{E}$ and using (4.7)-(4.12), we have

$$
\left.\left|\left\lfloor X_{H^{(m)}}\right\rceil\right|_{q, D_{p}\left(s_{m}, r_{m}\right)} \leq C(m), \| \partial_{\xi} X_{H^{(m)}}\right\rceil\left.\right|_{q, D_{p}\left(s_{m}, r_{m}\right)} \leq C(m) .
$$

Combining Lemma 7.2 and (7.17), we have

$$
\begin{aligned}
\left|\left\lfloor X_{\left\{H^{(m)}, F\right\}}\right\rceil\right|_{q, D_{p}\left(s_{m}^{5}, r_{m}^{5}\right)} & \leq \sup \left\|\left\lfloor\mathscr{D} X_{H^{(m)}}\right\rceil\right\|_{p, q}\left|X_{F}\right|_{p}+\left\|\left\lfloor\mathscr{D} X_{F}\right\rceil\right\|_{q, q}\left|\left\lfloor X_{H^{(m)}}\right\rceil\right|_{q} \\
& \leq C(m) K_{m}^{C} \varepsilon_{m}
\end{aligned}
$$

and

$$
\begin{aligned}
& \left.\| \partial_{\xi} X_{\left\{H^{(m)}, F\right\}}\right\rceil\left.\right|_{q, D_{p}\left(s_{m}^{5}, r_{m}^{5}\right)} \\
\leq & \sup \left(\left\|\left\lfloor\partial_{\xi} \mathscr{D} X_{H^{(m)}}\right\rceil\right\|_{p, q}\right)\left|\left\lfloor X_{F}\right\rceil\right|_{p}+\sup \left(\left\|\left\lfloor\mathscr{D} X_{H^{(m)}}\right\rceil\right\|_{p, q}\right)\left|\partial_{\xi} X_{F}\right|_{p} \\
& +\sup \left(\left\|\left\lfloor\partial_{\xi} \mathscr{D} X_{F}\right\rceil\right\|_{q, q}\right)\left|\left\lfloor X_{H^{(m)}}\right)\right|_{q}+\sup \left(\left\|\left\lfloor\mathscr{D} X_{F}\right\rceil\right\|_{q, q}\right)\left|\left\lfloor\partial_{\xi} X_{H^{(m)}}\right)\right|_{q} \\
\leq & C(m) K_{m}^{C} \varepsilon_{m},
\end{aligned}
$$

where the "sup" runs over $D_{p}\left(s_{m}^{5}, r_{m}^{5}\right) \times \mathscr{O}_{m+1}$. Repeating the last procedure, we have

$$
\begin{aligned}
\left\|\left\lfloor X_{\left\{\left\{H^{(m)}, F\right\}, F\right\}}\right\rceil\right\|_{q, D_{p}\left(s_{m+1}, r_{m+1}\right)} & \leq\left\|\left\lfloor\mathscr{D} X_{\left\{H^{(m)}, F\right\}}\right\rceil\right\|_{p, q} \mid\left\lfloor X_{F}\right\rceil\left\|_{p}+\right\|\left\lfloor\mathscr{D} X_{F}\right\rceil\left\|_{q, q}\right\|\left\lfloor X_{\left\{H^{(m)}, F\right\}}\right\rceil \|_{q} \\
& \leq C(m) K_{m}^{C} \varepsilon_{m}^{2}<\varepsilon_{m+1}
\end{aligned}
$$

and

$$
\begin{aligned}
& \quad\left|\left\lfloor\partial_{\xi} X_{\left\{\left\{H^{(m)}, F\right\}, F\right\}}\right\rceil\right|_{q, D_{p}\left(s_{m+1}, r_{m+1}\right)} \\
& \leq \sup \left(\left\|\left\lfloor\partial_{\xi} \mathscr{D} X_{\left\{H^{(m)}, F\right\}}\right\}\right\|_{p, q}\right)\left|\left\lfloor X_{F}\right\rceil\left\|_{p}+\sup \left(\left\|\left\lfloor\mathscr{D} X_{\left\{H^{(m)}, F\right\}}\right\}\right\|_{p, q}\right) \mid\left\lfloor\partial_{\xi} X_{F}\right\rceil\right\|_{p}\right. \\
& \quad+\sup \left(\|\left\lfloor\left\lfloor\partial_{\xi} \mathscr{D} X_{F}\right\rceil\|\|_{q, q}\right)\left|\left\lfloor X_{\left\{H^{(m)}, F\right\}}\right\rceil\right|_{q}+\sup \left(\left\|\left\lfloor\mathscr{D} X_{F}\right\rceil\right\| \|_{q, q}\right)\left|\left\lfloor\partial_{\xi} X_{\left\{H^{(m)}, F\right\}}\right\rceil\right|_{q}\right. \\
& \leq C(m) K_{m}^{C} \varepsilon_{m}^{2} \leq \varepsilon_{m+1},
\end{aligned}
$$

where the "sup" runs over $D_{p}\left(s_{m+1}, r_{m+1}\right) \times \mathscr{O}_{m+1}$. By applying (7.20), (7.21) and (7.13) to (5.51), we have

$$
\left.\| X_{[\underline{5.51}]}\right\rceil\left.\right|_{q, D_{p}\left(s_{m+1}, r_{m+1}\right) \times \mathscr{O}_{m+1}} \leq C(m+1) \varepsilon_{m+1}, \|\left.\left\lfloor\partial_{\xi} X_{[\underline{5.51]}]}\right\rceil\right|_{q, D_{p}\left(s_{m+1}, r_{m+1}\right) \times \mathscr{O}_{m+1}} \leq C(m+1) \varepsilon_{m+1} .
$$

By the definition of $\Gamma_{K_{m}}$, we have immediately

$$
\left|\left\lfloor X_{[5.49]}\right\rceil\right|_{q, D_{p}\left(s_{m+1}, r_{m+1}\right) \times \mathscr{O}_{m+1}} \leq C(m+1) \varepsilon_{m+1},\left|\left\lfloor\partial_{\xi} X_{[5.49}\right\rceil\right|_{q, D_{p}\left(s_{m+1}, r_{m+1}\right) \times \mathscr{O}_{m+1}} \leq C(m+1) \varepsilon_{m+1} .
$$

By (7.15), (7.16), (7.22) and (7.23), we have

$$
\left.\left\|\left.\left\lfloor X_{R^{(m+1)}}\right\rceil\right|_{q, D_{p}\left(s_{m+1}, r_{m+1}\right) \times \mathscr{O}_{m+1}} \leq C(m+1) \varepsilon_{m+1},\right\|\left\lfloor\partial_{\xi} X_{R^{(m+1)}}\right\rceil\right|_{q, D_{p}\left(s_{m+1}, r_{m+1}\right) \times \mathscr{O}_{m+1}} \leq C(m+1) \varepsilon_{m+1} .
$$

This proves $(3)_{l}$ with $l=m+1$ in Lemma4.1.

By a similar way,

$$
\left|\left\lfloor X_{P^{(m+1)}}\right\rceil\right|_{q, D_{p}\left(s_{m+1}, r_{m+1}\right) \times \mathscr{O}_{m+1}} \leq C(m+1),\left|\left\lfloor\partial_{\xi} X_{P^{(m+1)}}\right\rceil\right|_{q, D_{p}\left(s_{m+1}, r_{m+1}\right) \times \mathscr{O}_{m+1}} \leq C(m+1) .
$$


This proves $(4)_{l}$ with $l=m+1$ in Lemma 4.1 .

Finally, let us verify that $H_{0}^{(m+1)}, R^{(m+1)}$ and $P^{(m+1)}$ are real when $(x, y)$ are real and $\bar{z}$ is the complex conjugate of $z$ for $(x, y, z, \bar{z} ; \xi) \in D_{p}\left(s_{m+1}, r_{m+1}\right) \times \mathscr{O}_{m+1}$. Note that $R^{2 m}$ is real when $(x, y)$ are real and $\bar{z}$ is the complex conjugate of $z$. We assume $x$ is real until the end of this section. It follows that $R^{z \bar{z}}(x, \xi)$ is real symmetric operator and $R^{x}(x, \xi), R^{y}(x, \xi)$ are real vectors, and

$$
\overline{R^{z}(x, \xi)}=R^{\bar{z}}(x, \xi), \overline{R^{z z}(x, \xi)}=R^{\bar{z} \bar{z}}(x, \xi) .
$$

By (5.54), $F^{x}(x, \xi)$ is real. By (5.38) and (5.39), $\overline{R_{+}^{z}}=R_{+}^{\bar{z}}$. Note $B^{T}=\bar{B}$. Perform the complex conjugate "bar" in both sides of (5.57) and (5.58), we get

$$
\overline{F^{z}(x, \xi)}=F^{\bar{z}}(x, \xi) .
$$

Furthermore, by (5.37) and (7.26), we have that $R_{+}^{y}(x, \xi)$ is real. It follows from (5.55) that $F^{y}(x, \xi)$ is real. By (7.26) and (5.40), (5.41) and (5.42), we get

$$
\overline{R_{+}^{z z}(x, \xi)}=R_{+}^{\bar{z} \bar{z}}(x, \xi), \overline{R_{+}^{z \bar{z}}(x, \xi)}=R_{+}^{z \bar{z}}(x, \xi) .
$$

Noting (7.27) and performing the complex conjugate "bar" in both sides of (5.58), (5.59) and (5.60), we get

$$
\overline{F^{z z}(x, \xi)}=F^{\bar{z} \bar{z}}(x, \xi), \overline{F^{z \bar{z}}(x, \xi)}=F^{z \bar{z}}(x, \xi) .
$$

Consequently, $F$ defined by (5.22), (5.23) and (5.24) is real when $(x, y)$ are real and $\bar{z}$ is the complex conjugate of $z$. Arbitrarily take two Hamiltonian functions $F_{1}$ and $F_{2}$ defined by (5.22), (5.23) and (5.24). And assume $F_{1}$ and $F_{2}$ are real when $(x, y)$ are real and $z$ is the complex conjugate of $z$. Then it is easy to prove that the Poisson bracket $\left\{F_{1}, F_{2}\right\}$ is also real. It follows furthermore that $H_{0}^{(m+1)}, R^{(m+1)}$ and $P^{(m+1)}$ are real when $(x, y)$ are real and $\bar{z}$ is the complex conjugate of $z$ for $(x, y, z, \bar{z} ; \xi) \in D_{p}\left(s_{m+1}, r_{m+1}\right) \times \mathscr{O}_{m+1}$. This proves $(5)_{l}$ with $l=m+1$ in Lemma4.1.

By (5.6), (5.46) and (6.12), we have

$$
\sup _{\xi \in \mathscr{O}_{m+1}}\left|\partial_{\xi}^{t}\left(\widehat{R^{y}}(0, \xi)+\widehat{R_{+}^{y}}(0, \xi)\right)\right| \leq K^{C} \varepsilon_{m} \leq C(m) \varepsilon_{m}, t=0,1 .
$$

This proves $(1)_{l}$ with $l=m+1$ in Lemma4.1.

By (5.7), (5.45) and (6.22), we obtain

$$
\left\|\partial_{\xi}^{t}\left(\widehat{R^{z \bar{z}}}(0, \xi)+\widehat{R_{+}^{z \bar{z}}}(0, \xi)\right)\right\|_{h_{p} \rightarrow h_{q}} \leq K^{C} \varepsilon_{m} \leq C(m) \varepsilon_{m} .
$$

This proves $(2)_{l}$ with $l=m+1$ in Lemma4.1.

Up to now, we have verified all the assumptions are fulfilled for $l=m+1$. Thus the proof of the iterative lemma is complete.

\section{Proof of the main Theorems}

\section{Proof of Theorem 1.1.}

Let

$$
\begin{gathered}
\mathscr{O}=\cap_{j=0}^{\infty} \mathscr{O}_{j}, \\
\omega(\xi)=\omega^{0}+\sum_{m=1}^{\infty} \omega_{m}, \\
B^{z \bar{z}}=B_{0}^{z \bar{z}}+\sum_{m=1}^{\infty} B_{m}^{z \bar{z}} .
\end{gathered}
$$


And note

$$
D_{p}\left(s_{0}, r_{0}\right) \supset D_{p}\left(s_{0}, r_{0}\right) \supset \cdots D_{p}\left(s_{m}, r_{m}\right) \supset \cdots \supset D_{p}\left(s_{0} / 2, r_{0} / 2\right) .
$$

Using the iterative lemma and let $m \rightarrow \infty$, the proof of the main theorem is finished in the standard procedure in KAM theory. Here we omit the detail.

Proof of Corollary 1 Since $\mathscr{T}_{0}$ is invariant for the flow of $X_{H^{\infty}}$, the pull-back tori $\Phi\left(\mathscr{T}_{0}\right)$ is invariant for the flow of the original Hamiltonian vector field $\Phi^{*} X_{H^{\infty}}=X_{H}$, too.

Proof of Corollary 2] Consider a linear Hamiltonian system with Hamiltonian

$$
H=\sum_{j \in \mathbb{Z}^{d}} \lambda_{j} z_{j} \bar{z}_{j}+\left\langle B^{0}(\xi) z, \bar{z}\right\rangle+\left\langle R^{z z}(x, \xi) z, z\right\rangle+\left\langle R^{z \bar{z}}(x, \xi) z, \bar{z}\right\rangle+\left\langle R^{\bar{z} \bar{z}}(x, \xi) \bar{z}, \bar{z}\right\rangle
$$

where $(x, y, z, \bar{z}) \in D_{p}\left(s_{0} / 2, r_{0} / 2\right)$ and $\xi \in \mathscr{O}$. Using Theorem 1.1, there is a symplectic transformation such that $H$ is reduced to

$$
H^{\infty}=\left\langle\left(\Lambda+B^{\infty}(\xi)\right) z, \bar{z}\right\rangle
$$

by digging a subset of $\mathscr{O}$ with small Lebesgue measure. Multiplying $\bar{z}$ in both sides of the equation

$$
\dot{z}=\mathbf{i}\left(\Lambda+B^{\infty}(\xi)\right) z, \quad z \in h_{p},
$$

one gets

$$
\sum_{j \in \mathbb{Z}^{d}} \dot{z}_{j} \bar{z}_{j}=\mathbf{i}\left\langle\left(\Lambda+B^{\infty}(\xi)\right) z, \bar{z}\right\rangle
$$

Recall that $\left\langle\left(\Lambda+B^{\infty}(\xi)\right) z, \bar{z}\right\rangle$ is real for any $z \in h_{p}$, when $\bar{z}$ is the complex conjugate of $z$. Write $z=\left(|j|^{p} w_{j}: j \in \mathbb{Z}^{d}\right)$ where $w=\left(w_{j}: j \in \mathbb{Z}^{d}\right) \in \ell_{2}$. So one gets

$$
\frac{1}{2} \frac{d}{d t} \sum_{j \in \mathbb{Z}^{d}}|j|^{2 p}\left|w_{j}\right|^{2}=\frac{1}{2} \frac{d}{d t} \sum_{j \in \mathbb{Z}^{d}}\left|z_{j}(t)\right|^{2}=\sum_{j \in \mathbb{Z}^{d}} \Re \dot{z}_{j} \bar{z}_{j}=0, \text { while } z(t) \in h_{p} .
$$

It follows that $\|z(t)\|_{p} \equiv$ constant. This completes the proof.

Proof of Theorem 1.2. In order to distinguish the normal frequencies $\lambda_{j}$ 's in Theorem 1.1 and the $\lambda_{j}$ 's in Theorem 1.2. we denote by $\lambda_{j}^{\prime}$ the normal frequencies $\lambda_{j}$ 's in Theorem 1.2 By Assumption $\mathbf{B}^{\star}$, we can write

$$
\lambda_{j}^{\prime}=\varpi+\lambda_{j}, \quad j \in \mathbb{Z}^{d}
$$

where $\lambda_{j}$ 's satisfy Assumption B. Thus the homological equation 2.9) should be replaced by

$$
(\varpi-(k, \omega)+\Lambda+B) \widehat{F}(k)=\widehat{R}(k), \quad \forall k \in \mathbb{Z}^{N}, 0<|k| \leq K,
$$

where $\Lambda=\operatorname{diag}\left(\lambda_{j}: j \in \mathbb{Z}^{d}\right)$ and $\varpi-(k, \omega)=(\varpi-(k, \omega)) E$ with $E$ being an identity from $h_{p}$ to $h_{p}$. (at this time, $p=q$.) Moreover, 2.10) should be replaced by

$$
|\varpi-(k, \omega(\xi))| \geq K^{-c_{21}}, \quad 0<|k| \leq K, \xi \in \mathscr{O} \backslash \mathscr{O}_{1}
$$

And (3.10) should be replaced by

$$
((k, \omega) \pm(\varpi+\Lambda+B)) \widehat{F}(k) \pm \widehat{F}(k)(\varpi+\Lambda+\breve{B})=\widehat{R}(k), \quad k \in \mathbb{Z}^{N},|k| \leq K
$$

Moreover, (8.4) should be replaced by

$$
\left|((k, \omega) \pm \varpi) \pm \lambda_{j}\right| \geq \frac{1}{2} K^{-c}, \quad \text { for } k \in \mathbb{Z}^{N}, 0<|k| \leq K, j \in \mathbb{Z}^{d}, \xi \in \mathscr{O} \backslash \mathscr{O}_{4}
$$

After finishing the replacements as above, we can construct Lemmas 2.1 and 3.1 for theorem 1.2 . The remaining proof for theorem 1.2 is similar to that of Theorem 1.1 We omit it here. 


\section{Application to Benjamin-Bona-Mahony (BBM) equation}

Benjamin-Bona-Mahony (BBM) equation: was studied in 1972 by Benjamin, Bona, and Mahony[6] as an improvement of the Korteweg-de Vries equation (KdV equation) for modeling long surface gravity waves of small amplitude - propagating uni-directionally in $1+1$ dimensions. Also see [1], [26] and [9] for the related topics. Consider BBM equation subject to periodic boundary condition

$$
u_{t}-u_{x x t}+u_{x}+u u_{x}=0, \quad u(t, 0)=u(t, T) .
$$

This equation can be written as a Hamiltonian system

$$
u_{t}=-\left(1-\partial_{x x}\right)^{-1} \partial_{x} \nabla_{u} H(u)
$$

with Hamiltonian function

$$
H(u)=\frac{1}{2} \int_{0}^{T} u^{2} d x+\frac{1}{6} \int_{0}^{T} u^{3} d x
$$

and the symplectic structure $-\left(1-\partial_{x x}\right)^{-1} \partial_{x}$ and the working space

$$
u \in \mathscr{H}_{0}^{p_{0}}=\left\{u \in \mathscr{H}^{p_{0}}(\mathbb{T}: \mathbb{R}): \int_{0}^{T} u d x=0\right\},
$$

where $\mathscr{H}^{p_{0}}(\mathbb{T})$ is the usual Sobolev space with some $p_{0}>0$.

Let $\overline{\mathbb{Z}}=\mathbb{Z} \backslash\{0\}$ and set $\tau=\frac{2 \pi}{T}$. Denote by $h_{p_{0}}$ the discretization of $\mathscr{H}_{0}^{p_{0}}$, i.e.

$$
h_{p_{0}}:=\left\{z=\left(z_{j} \in \mathbb{C}\right):\|z\|_{p}^{2}=\sum_{j \in \overline{\mathbb{Z}}}\left|z_{j}\right|^{2} j^{2 p_{0}}<\infty\right\} .
$$

Make Fourier transform $\mathscr{F}: u \mapsto z=\left(z_{j} \in \mathbb{C}: j \in \overline{\mathbb{Z}}\right)$ by

$$
u=\sum_{j \in \overline{\mathbb{Z}}} \delta_{j} z_{j} \phi_{j}, \quad \phi_{j}=\frac{1}{\sqrt{T}} e^{\mathbf{i} \tau j \cdot x}, \quad \delta_{j}=\sqrt{\frac{\tau|j|}{1+\tau^{2} j^{2}}} .
$$

Note that $\bar{z}_{j}=z_{-j}$ if and only if $u \in \mathbb{R}$. Then $\mathscr{F}$ is isometry from $\mathscr{H}^{p_{0}}$ to $h_{p_{0}+\frac{1}{2}}=h_{p}$ (with $p_{0}+1 / 2=p$ ) and (9.2) is changed into a Hamiltonian system with its symplectic structure $-\mathbf{i} \sum_{j \geq 1} d z_{j} \wedge d z_{-j}$ :

$$
\mathbf{i} \dot{z}_{j}=\frac{\partial H}{\partial \bar{z}_{j}}, \quad-\mathbf{i} \dot{\bar{z}}_{j}=\frac{\partial H}{\partial z_{j}}, \quad \bar{z}_{j}=z_{-j},
$$

where

$$
H(z, \bar{z})=\sum_{j \geq 1} \lambda_{j} z_{j} z_{-j}+\frac{1}{6 \sqrt{T}} \sum_{j+k+l=0, j, k, l \in \overline{\mathbb{Z}}} \delta_{j} \delta_{k} \delta_{l} z_{j} z_{k} z_{l}:=H_{0}+R
$$

and

$$
\lambda_{j}=\frac{\tau j}{1+\tau^{2} j^{2}}, \quad\left|\lambda_{j}\right| \approx|j|^{-\kappa}, \quad \kappa=1
$$

Let

$$
\Lambda=\operatorname{diag}\left(\lambda_{j}: j \in \overline{\mathbb{Z}}\right)
$$

and

$$
R=\sum_{j+k+l=0, j, k, l \in \overline{\mathbb{Z}}} R_{j k l} z_{j} z_{k} z_{l}, \quad G_{j k l}=\frac{\delta_{j} \delta_{k} \delta_{l}}{6 \sqrt{T}} .
$$

By $X_{R}$ denote the Hamiltonian vector filed of $R$ with symplectic structure $-\mathbf{i} \sum_{j \geq 1} d z_{j} \wedge d z_{-j}$ :

$$
X_{R}=\left(\mathbf{i} \sigma_{j} \frac{\partial R}{\partial z_{-j}}: j \in \overline{\mathbb{Z}}\right), \sigma_{j}=\operatorname{sign} j .
$$


Lemma 9.1. The function $H(z, \bar{z})$ is real when $\bar{z}_{j}$ is the complex conjugate of $z_{j}$ for each $j \in \overline{\mathbb{Z}}$. The Hamiltonian vector field $X_{R}$ of the perturbation $R$ is analytic from $h_{p}$ to $h_{q}$ with $q=p+\kappa$ and $\kappa=1$. Moreover,

$$
\left\|\left\lfloor X_{R}\right\rceil\right\|_{q} \leq C\|z\|_{p}^{2}
$$

Proof. Observe that $\delta_{j} \approx|j|^{-1 / 2}$ and note that

$$
\frac{\partial R}{\partial z_{-j}}=3 \delta_{j} \sum_{k+l=j} \delta_{k} \delta_{l} z_{k} z_{l}
$$

Hence,

$$
\left|\frac{\partial R}{\partial z_{-j}}\right| \leq 3 \delta_{j} \sum_{k+l=j} \delta_{k} \delta_{l}\left|z_{k}\right|\left|z_{l}\right|=3 \delta_{j} \cdot(w * w)_{j},
$$

where $w=\left(w_{j}: j \in \overline{\mathbb{Z}}\right)$ with $w_{j}=\left|\delta_{j} z_{j}\right|$ and $w * w$ is the convolution of $w$ and $w$. It follows that

$$
\left\|\left\lfloor X_{R}\right\rceil\right\|_{q} \leq\|w * w\|_{q-\frac{1}{2}} \leq C\|w\|_{q-\frac{1}{2}}^{2}=C\|w\|_{p+\frac{1}{2}}^{2}=C\|z\|_{p}^{2}
$$

The remaining statements are obvious.

Fix two integers $N$ and $\tilde{N}$ with $0<N \leq \tilde{N}$. Let

$$
J=\left\{1 \leq j_{1}<j_{t}<\cdots<j_{N} \leq \tilde{N}: j_{t} \in \mathbb{N} \text { for } 1 \leq t \leq N\right\} .
$$

Split $z=\left(z_{j}\right)_{j \in \overline{\mathbb{Z}}}=(\tilde{z}, \hat{z})$ with $\tilde{z}=\left(z_{j_{1}}, \cdots, z_{j_{N}}, z_{-j_{1}}, \cdots, z_{-j_{N}}\right)$ and $\hat{z}=z \ominus \tilde{z}$.

Lemma 9.2. Assume the number $\tau=2 \pi / T$ is transcendental. There exists a real analytic symplectic coordinate transformation $\Phi$ which maps the neighborhood of the origin of $h_{p}$ to $h_{p}$ such that the Hamiltonian $H$ defined by (9.6) is changed into a partial Birkhoff normal form up to order four. More precisely,

$$
H \circ \Phi=H_{0}+\bar{G}+\hat{G}+\tilde{R},
$$

where

$$
\bar{G}=\sum_{k, l \geq 1,\{k, l\} \cap J \neq \emptyset} \bar{G}_{k l}\left|z_{k}\right|^{2}\left|z_{l}\right|^{2}
$$

with

$$
\bar{G}_{k l}= \begin{cases}-\frac{1}{T} \frac{\tau^{2} k l}{\left[\tau^{2}\left(k^{2}+k l+l^{2}\right)+3\right]\left[\tau^{2}\left(k^{2}-k l+l^{2}\right)+3\right]}, & k \neq l, \\ \frac{1}{12 T} \frac{1}{\tau^{2} k^{2}+1}, & \text { otherwise },\end{cases}
$$

and

$$
\left\|\left\lfloor X_{\hat{G}}\right\rceil\right\|_{q}=O\left(\|\hat{z}\|_{p}^{3}\right), \quad\left\|\left\lfloor X_{\tilde{R}}\right\rceil\right\|_{q}=O\left(\|z\|_{p}^{4}\right) .
$$

In order to prove the last lemma, we need the following lemmas.

Lemma 9.3. Assume the number $\tau=2 \pi / T$ is transcendental. (1) For any $j, k, l \in \overline{\mathbb{Z}}$ with $j+k+l=0$, one has

$$
\lambda_{j}+\lambda_{k}+\lambda_{l} \neq 0
$$

(2) For any $j, k, l, m \in \overline{\mathbb{Z}}$ with $j+k+l+m=0$ and $(j+k)(j+l)(j+m) \neq 0$, one has

$$
\lambda_{j}+\lambda_{k}+\lambda_{l}+\lambda_{m} \neq 0
$$


Proof. Recall $\lambda_{j}=\frac{\tau j}{1+\tau^{2} j^{2}}$ for all $j \in \overline{\mathbb{Z}}$. In view of $j+k+l=0$, by calculation,

$$
\lambda_{j}+\lambda_{k}+\lambda_{l}=-\left(\frac{j k l \tau^{3}\left(3+\left(k^{2}+k l+l^{2}\right) \tau^{2}\right)}{\left(1+j^{2} \tau^{2}\right)\left(1+k^{2} \tau^{2}\right)\left(1+l^{2} \tau^{2}\right)}\right) .
$$

That is,

$$
\lambda_{j}+\lambda_{k}+\lambda_{l}= \pm \delta_{j}^{2} \delta_{k}^{2} \delta_{l}^{2}\left(3+\tau^{2}\left(k^{2}+k l+l^{2}\right)\right) .
$$

Noting $k^{2}+k l+l^{2}>0$. It follows $\lambda_{j}+\lambda_{k}+\lambda_{l}>0$.

When $j+k+l+m=0$, by calculation one has that

$$
\begin{aligned}
& \tau^{-3}\left(\left(1+j^{2} \tau^{2}\right)\left(1+k^{2} \tau^{2}\right)\left(1+l^{2} \tau^{2}\right)\left(1+m^{2} \tau^{2}\right)\right)\left(\lambda_{j}+\lambda_{k}+\lambda_{l}+\lambda_{m}\right) \\
& =3(k+l)(k+m)(l+m)+(k+l)(k+m)(l+m)\left(k^{2}+l^{2}+l m+m^{2}+k(l+m)\right) \tau^{2} \\
& \quad+k l(k+l) m(k+m)(l+m)(k+l+m) \tau^{4} .
\end{aligned}
$$

Since $k l(k+l)(k+m)(l+m) \neq 0$ and that $\tau$ is transcendental, $\lambda_{j}+\lambda_{k}+\lambda_{l}+\lambda_{m} \neq 0$.

Lemma 9.4. Assume the number $\tau=2 \pi / T$ is transcendental. Set

$$
\begin{gathered}
\Delta_{3}=\left\{(j, k, l) \in \overline{\mathbb{Z}}^{3}: j+k+l=0, \min \{|j|,|k|,|l|\} \leq \tilde{N}\right\}, \\
\Delta_{4}=\left\{(j, k, l, m) \in \overline{\mathbb{Z}}^{4}: j+k+l+m=0,(j+k)(j+l)(j+m) \neq 0, \min \{|j|,|k|,|l|,|m|\} \leq \tilde{N}\right\} .
\end{gathered}
$$

Then there exists a constant $C=C(\tilde{N})>0$ depending on only $\tilde{N}$ such that

$$
\inf _{(j, k, l) \in \Delta_{3}}\left|\lambda_{j}+\lambda_{k}+\lambda_{l}\right| \geq C(\tilde{N})
$$

and

$$
\inf _{(j, k, l, m) \in \Delta_{4}}\left|\lambda_{j}+\lambda_{k}+\lambda_{l}+\lambda_{m}\right| \geq C(\tilde{N}) .
$$

Proof. We give the proof only for (9.16) with $|j| \leq \tilde{N}$ and $|k| \leq \tilde{N}$ and $l \in \overline{\mathbb{Z}}$. The others are similar. Clearly, there is a constant $C_{2}=C_{2}(\tilde{N})>0$ such that

$$
\inf _{\Delta_{2}}\left|\lambda_{j}+\lambda_{k}\right| \geq C_{2}(\tilde{N}), \text { here } \Delta_{2}=\left\{(j, k) \in \overline{\mathbb{Z}}^{2}: j+k \neq 0, \max \{|j|,|k|\} \leq \tilde{N}\right\} .
$$

Note $\lim _{|l| \rightarrow \infty} \lambda_{l}=0$. So there is a constant $M=M(\tilde{N})>0$ depending on $\tilde{N}$ such that $\left|\lambda_{l}\right| \leq C_{2}(\tilde{N}) / 4$ when $|l| \geq M(\tilde{N})$. Thus, for $|l| \geq M(\tilde{N})$,

$$
\inf _{(j, k, l) \in \Delta_{3}}\left|\lambda_{j}+\lambda_{k}+\lambda_{l}\right|>\inf _{(j, k) \in \Delta_{2}}\left|\lambda_{j}+\lambda_{k}\right|-\frac{C_{2}(\tilde{N})}{4} \geq \frac{C_{2}(\tilde{N})}{2} .
$$

When $|j| \leq \tilde{N},|k| \leq \tilde{N}$ and $|l| \leq M(\tilde{N})$, using Lemma9.3, there is a constant $\tilde{C}_{3}(\tilde{N})=\tilde{C}_{3}(\tilde{N}, M(\tilde{N}))>0$ such that

$$
\inf _{(j, k, l) \in \Delta_{3}}\left|\lambda_{j}+\lambda_{k}+\lambda_{l}\right| \geq \inf _{j+k+l=0,|j|+|k|+|l| \leq 2 \tilde{N}+M(\tilde{N})}\left|\lambda_{j}+\lambda_{k}+\lambda_{l}\right| \geq \tilde{C}_{3}(\tilde{N}) .
$$

The inequality (9.16) with $|j| \leq \tilde{N},|k| \leq \tilde{N}$ and $l \in \overline{\mathbb{Z}}$ is proved by letting

$$
C(\tilde{N})=\min \left\{C_{2}(\tilde{N}) / 2, \tilde{C}_{3}(\tilde{N})\right\} .
$$


Proof of Lemma 9.1, Let $\Psi^{(3)}=X_{F^{(3)}}^{1}$ be the time-1-map of the flow of the hamiltonian vector field $X_{F^{(3)}}$ given by the hamiltonian

$$
F^{(3)}=\sum_{j, k, l \in \overline{\mathbb{Z}}, j+k+l=0} F_{j k l}^{(3)} z_{j} z_{k} z_{l}
$$

with coefficients

$$
\mathbf{i} F_{j k l}^{(3)}= \begin{cases}\frac{R_{j k l}}{\lambda_{j}+\lambda_{k}+\lambda_{l}}, & j+k+l=0, \\ 0, & \text { otherwise. }\end{cases}
$$

Recall that $\lambda_{j}+\lambda_{k}+\lambda_{l} \neq 0$, when $j+k+l=0$. So $F_{j k l}^{(3)}$ is well defined in the last formula.

By (9.15) and (9.9) we have that the $j$-th entry of the vector field $X_{F^{(3)}}$ is

$$
\pm \mathbf{i} \frac{\partial F^{(3)}}{\partial z_{-j}}=\frac{1}{2 \sqrt{T} \delta_{j}} \sum_{k+l=j} \frac{z_{k} z_{l}}{\delta_{k} \delta_{l}\left(\tau^{2}\left(k^{2}+l^{2}+k l\right)+3\right)} .
$$

Define a vector field $\left\lfloor X_{F^{(3)}}\right\rceil$ with its $j$-th entry being

$$
\left\lfloor X_{F^{(3)}}\right\rceil_{j}=\frac{1}{2 \sqrt{T} \delta_{j}} \sum_{k+l=j} \frac{\left|z_{k}\right|\left|z_{l}\right|}{\delta_{k} \delta_{l}\left(\tau^{2}\left(k^{2}+l^{2}+k l\right)+3\right)} .
$$

Observe that

$$
k^{2}+k l+l^{2}=(k+l)^{2}-k l \geq \frac{1}{2}(k+l)^{2}=\frac{1}{2} j^{2} .
$$

So

$$
\left\lfloor X_{F^{(3)}}\right\rceil_{j} \leq C|j|^{-3 / 2} \sum_{k+l=j} \sqrt{|k||l|}\left|z_{k}\right|\left|z_{l}\right| .
$$

Let $w=\left(w_{k}: k \in \mathbb{Z}^{d}\right)$ with $w_{k}=\sqrt{|k|}\left|z_{k}\right|$. Then, noting $q=p+1$,

$$
\left.\left\|\left\lfloor X_{F^{(3)}}\right\rceil\right\|\right|_{q} \leq C\|w * w\|_{p-\frac{1}{2}} \leq C\|w\|_{p-\frac{1}{2}}^{2}=C\|z\|_{p}^{2} .
$$

Therefore $X_{F^{(3)}}$ is a real analytic vector field which maps a small neighborhood of the origin in $h_{p}$ to $h_{q}$. And hence $\Psi^{(3)}$ is a real analytic, symplectic change of coordinates defined at least in a neighborhood of the origin in $h_{p}$.

Expanding at $t=0$ and using Taylor's formula we have

$$
\begin{aligned}
H \circ \Psi^{(3)}= & \left.H \circ X_{F^{(3)}}^{t}\right|_{t=1} \\
= & H+\left\{H, F^{(3)}\right\}+\int_{0}^{1}(1-t)\left\{\left\{H, F^{(3)}\right\}, F^{(3)}\right\} \circ X_{F^{(3)}}^{t} d t \\
= & H_{0}+R+\left\{H_{0}, F^{(3)}\right\} \\
& +\left\{R, F^{(3)}\right\}+\int_{0}^{1}(1-t)\left\{\left\{H, F^{(3)}\right\}, F^{(3)}\right\} \circ X_{F^{(3)}}^{t} d t .
\end{aligned}
$$

By calculation, we obtain

$$
R+\left\{H_{0}, F^{(3)}\right\}=0 .
$$

Again using Taylor's formula in the last term in (9.18) and noting (9.19), we have

$$
H \circ \Psi^{(3)}=H_{0}+\frac{1}{2}\left\{R, F^{(3)}\right\}+\frac{1}{2} \int_{0}^{1}\left(1-t^{2}\right)\left\{\left\{R, F^{(3)}\right\}, F^{(3)}\right\} \circ X_{F^{(3)}}^{t} d t .
$$

By direct calculation, we have

$$
\begin{aligned}
R^{4}: & =\frac{1}{2}\left\{R, F^{(3)}\right\} \\
& =\frac{1}{2} \sum_{j \in \overline{\mathbb{Z}}} \frac{\partial R}{\partial z_{j}}\left(-\mathbf{i} \sigma_{j}\right) \frac{\partial F^{(3)}}{\partial z_{-j}} \\
& =\frac{1}{8 T} \sum_{k+l+m+n=0}^{\prime} \frac{\delta_{k} \delta_{l}}{\sigma_{m} \sigma_{n} \delta_{m} \delta_{n}\left(\tau^{2}\left(m^{2}+m n+n^{2}\right)+3\right)} z_{k} z_{l} z_{m} z_{n} \\
& :=\sum_{k+l+m+n=0}^{\prime} R_{k l m n} z_{k} z_{l} z_{m} z_{n},
\end{aligned}
$$


where $\sum_{k, l, m, n}^{\prime}$ runs over the set $\left\{(k, l, m, n) \in \overline{\mathbb{Z}}^{4}\right\}$ and $\sigma_{k}$ is the sign of $k$, i.e. $\sigma_{k}=1$ if $k>0$ and $\sigma_{k}=-1$ if $k<0$. So the $j$-th entry of the vector field $X_{R^{4}}$ is

$$
\begin{aligned}
\pm \mathbf{i} \frac{\partial R^{4}}{\partial_{z_{-j}}}= & \delta_{j} \sum_{l+m+n=j}^{\prime} \frac{\delta_{l}}{4 T \sigma_{m} \sigma_{n} \delta_{m} \delta_{n}\left(\tau^{2}\left(m^{2}+m n+n^{2}\right)+3\right)} z_{l} z_{m} z_{n} \\
& +\sum_{k+l+m=j}^{\prime} \frac{\delta_{k} \delta_{l}}{4 T \sigma_{m} \sigma_{j} \delta_{m} \delta_{j}^{2}\left(\tau^{2}\left(m^{2}-m j+j^{2}\right)+3\right)} z_{k} z_{l} z_{m}
\end{aligned}
$$

Note that

$$
m^{2}+m n+n^{2}+3 \geq|m n|, m, n \in \overline{\mathbb{Z}} .
$$

Then

$$
\left|4 T \sigma_{m} \sigma_{n} \delta_{m} \delta_{n}\left(\tau^{2}\left(m^{2}+m n+n^{2}\right)+3\right)\right| \geq \frac{1}{C} \sqrt{|m||n|}
$$

and

$$
\left|4 T \sigma_{m} \sigma_{j} \delta_{m} \delta_{j}^{2}\left(\tau^{2}\left(m^{2}-m j+j^{2}\right)+3\right)\right| \geq \frac{1}{C} \sqrt{|m|}
$$

Thus,

$$
\left| \pm \mathbf{i} \frac{\partial R^{4}}{\partial_{-z_{j}}}\right| \leq C \frac{1}{\sqrt{|j|}} \sum_{l+m+n=-j}^{\prime} \frac{\left|z_{l}\right|}{\sqrt{|l|}} \frac{\left|z_{m}\right|}{\sqrt{|m|}} \frac{\left|z_{n}\right|}{\sqrt{|n|}} .
$$

Let $w=\left(\frac{\left|z_{l}\right|}{\sqrt{|l|}}: l \in \overline{\mathbb{Z}}\right)$. Then

$$
\left\|\left\lfloor X_{R^{4}}\right\rceil\right\|_{q}=\left\|\left\lfloor X_{R^{4}}\right\rceil\right\|_{p+1} \leq\|w * w * w\|_{p+\frac{1}{2}} \leq\|w\|_{p+\frac{1}{2}}^{3}=\|z\|_{p}^{3} .
$$

Let

$$
\begin{gathered}
\Xi_{\leq}=\left\{(k, l, m, n) \in \overline{\mathbb{Z}}^{4}: k+l+m+n=0,(k+l)(k+m)(l+m)=0, J \cap\{|k|,|l|,|m|,|n|\} \neq \emptyset\right\}, \\
\Xi_{>}=\left\{\left\{(k, l, m, n) \in \overline{\mathbb{Z}}^{4}: k+l+m+n=0, J \cap\{|k|,|l|,|m|,|n|\}=\emptyset\right\} .\right.
\end{gathered}
$$

Then we can write

$$
\begin{aligned}
R^{4} & =\sum_{k, l, m, n}^{1} R_{k l m n} z_{k} z_{l} z_{m} z_{n} \\
& =\sum_{\Xi_{\leq}} R_{k l m n} z_{k} z_{l} z_{m} z_{n}+\sum_{\Delta_{4}} R_{k l m n} z_{k} z_{l} z_{m} z_{n}+\sum_{\Xi_{>}} R_{k l m n} z_{k} z_{l} z_{m} z_{n} \\
& :=R_{(1)}^{4}+R_{(2)}^{4}+R_{(3)}^{4} .
\end{aligned}
$$

By direct calculation,

$$
R_{(1)}^{4}=\sum_{\Xi_{\leq}} R_{k l m n}=\sum_{k \geq 1, l \geq 1} \bar{G}_{k l}\left|z_{k}\right|^{2}\left|z_{l}\right|^{2}
$$

where $\bar{G}_{k l}$ is defined by $(9.13)$. Let $X_{F^{(4)}}^{t}$ be the flow of the Hamiltonian vector field with Hamiltonian $F^{(4)}$ :

$$
F^{(4)}=\sum_{k, l, m, n \in \overline{\mathbb{Z}}} F_{k l m n}^{4} z_{k} z_{l} z_{m} z_{n}
$$

where

$$
\mathbf{i} F_{k l m n}^{4}= \begin{cases}\frac{1}{\lambda_{k}+\lambda_{l}+\lambda_{m}+\lambda_{n}} R_{k l m n}, & (k, l, m, n) \in \Delta_{4} \\ 0, & \text { otherwise }\end{cases}
$$

By (9.17), we have

$$
\left|\mathbf{i} F_{k l m n}^{4}\right| \leq C\left|R_{k l m n}\right|
$$


By (9.25) and 9.21,

$$
\left.\left\|\left\lfloor X_{F^{(4)}}\right\rceil\right\|_{q} \leq C \mid \| X_{R^{4}}\right\rceil\left\|_{q} \leq C|| z\right\|_{p}^{3}
$$

Let $\Psi^{(4)}=\left.X_{F^{(4)}}^{t}\right|_{t=1}$. Thus,

$$
\begin{aligned}
H \circ \Psi^{(3)} \circ \Psi^{(4)}= & H_{0}+\left\{H_{0}, F^{(4)}\right\}+R^{4} \\
& +\int_{0}^{1}(1-t)\left\{\left\{H_{0}, F^{(4)}\right\}, F^{(4)}\right\} \circ X_{F^{(4)}}^{t} d t \\
& +\int_{0}^{1}\left\{R^{4}, F^{(4)}\right\} \circ X_{F^{(4)}}^{t} d t \\
& +\left(\int_{0}^{1}\left(1-t^{2}\right)\left\{\left\{R, F^{(3)}\right\}, F^{(3)}\right\} \circ X_{F^{(3)}}^{t} d t\right) \circ \Psi^{(4)} .
\end{aligned}
$$

By direct calculation,

$$
\left\{H_{0}, F^{(4)}\right\}+R^{4}=R_{(1)}^{4}+R_{(3)}^{4}=\bar{G}+O\left(\|\hat{z}\|_{p}^{4}\right) .
$$

Note that all of $\left\lfloor X_{R}\right\rceil,\left\lfloor X_{R^{3}}\right\rceil,\left\lfloor X_{R^{4}}\right\rceil,\left\lfloor X_{F^{(3)}}\right\rceil,\left\lfloor X_{F^{(4)}}\right\rceil$ are in $h_{q}$. It is easy to verify that $\overline{9.28]}=O\left(\|z\|_{p}^{6}\right)$ and $(9.29)=O\left(\|z\|_{p}^{6}\right)$ and $[9.30)=O\left(\|z\|_{p}^{5}\right)$. The proof of Lemma 9.2 is finished.

Restrict $|\tilde{z}| \leq \varepsilon_{0}^{1 / 4}$ and $\|\hat{z}\|_{p} \leq \varepsilon_{0}^{1 / 3}$. Then

$$
\left\|\left\lfloor X_{\hat{G}+\tilde{R}}\right\rceil\right\|_{q} \leq C\left(|\tilde{z}|^{4}+\|\hat{z}\|_{p}^{3}\right) \leq C \varepsilon_{0} .
$$

Introduce action-angle variables $(y, x)$ by

$$
\begin{cases}z_{j_{k}}=\sqrt{\zeta_{k}+y_{k}} e^{-\mathbf{i} x_{k}}, & z_{-j_{k}}=\sqrt{\zeta_{k}+y_{k}} e^{\mathbf{i} x_{k}}, k=1, \ldots, n, j_{k} \in J, \\ z_{j}=z_{j}, & z_{-j}=z_{-j}, j \in \overline{\mathbb{Z}} \backslash J,\end{cases}
$$

where $\zeta=\left(\zeta_{1}, \ldots, \zeta_{n}\right) \in \mathbb{R}_{+}^{n}$ and $\varepsilon_{0}^{1 / 2}<|\zeta| \leq 2 \varepsilon_{0}^{1 / 2}$. Then

$$
H_{0}=\sum_{1 \leq k \leq N} \frac{\tau j_{k}}{1+\tau^{2} j_{k}^{2}}\left(\zeta_{k}+y_{k}\right)+\sum_{\mathbb{Z} \backslash J} \frac{\tau^{2} j}{1+\tau^{2} j^{2}} z_{j} z_{-j}
$$

and

$$
\begin{aligned}
\bar{G}= & \sum_{1 \leq k, l \leq N} \bar{G}_{j_{k} l_{k}}\left(\zeta_{k}+y_{k}\right)\left(\zeta_{l}+y_{l}\right)+\sum_{1 \leq k \leq N, l \in \overline{\mathbb{Z}} \backslash J} \bar{G}_{j_{k} l}\left(\zeta_{k}+y_{k}\right) z_{l} z_{-l} \\
& +\sum_{k \in \overline{\mathbb{Z}} \backslash J, 1 \leq l \leq N} \bar{G}_{k j_{l}}\left(\zeta_{l}+y_{l}\right) z_{k} z_{-k} .
\end{aligned}
$$

Thus, up to a constant depending on $\zeta$,

$$
H_{0}+\bar{G}=\sum_{1 \leq j \leq N} \omega_{j}^{0}(\zeta) y_{j}+\sum_{j \in \overline{\mathbb{Z}} \backslash J} \Omega_{j}^{0}(\zeta) z_{j} z_{-j}
$$

where

$$
\omega^{0}(\zeta)=\lambda^{(N)}+\mathscr{B} \zeta, \quad \Omega^{0}(\zeta)=\lambda^{\infty}+S \zeta
$$

with

$$
\lambda^{(N)}=\left(\frac{\tau j_{1}}{1+\tau^{2} j_{1}^{2}}, \ldots, \frac{\tau j_{N}}{1+\tau^{2} j_{N}^{2}}\right), \quad \lambda^{\infty}=\left(\frac{\tau j}{1+\tau^{2} j^{2}}: j \in \overline{\mathbb{Z}} \backslash J\right)
$$


and $\mathscr{B}=\left(\mathscr{B}_{k l}: k, l \in J\right)$ with matrix elements

$$
\mathscr{B}_{k l}= \begin{cases}-\frac{2}{T} \frac{\tau^{2} j_{k} j_{l}}{\left(\tau^{2}\left(j_{k}^{2}+j_{k} j_{l}+j_{l}^{2}\right)+3\right)\left(\tau^{2}\left(j_{k}^{2}-j_{k} j_{l}+j_{l}^{2}\right)+3\right)}, & k \neq l, \\ \frac{1}{6 T} \frac{1}{\tau^{2} j_{k}^{2}+1}, & k=l,\end{cases}
$$

and $S=\left(S_{k l}: k \in \overline{\mathbb{Z}} \backslash J, l \in J\right)$ with matrix elements

$$
S_{k l}=-\frac{2}{T} \frac{\tau^{2} k l}{\left(\tau^{2}\left(k^{2}+k l+l^{2}\right)+3\right)\left(\tau^{2}\left(k^{2}-k l+l^{2}\right)+3\right)} .
$$

Let $M=\operatorname{diag}\left(\frac{1}{6 T} \frac{1}{\tau^{2} j_{k}^{2}+1}: k=1, \ldots, N, j_{k} \in J\right)$. Clearly, there does exist $M^{-1}$ and the matrix elements of the matrix $M^{-1} \mathscr{B}$ are

$$
\left(M^{-1} \mathscr{B}\right)_{k l}= \begin{cases}-3 \frac{\left(\tau^{2} j_{k}^{2}+1\right)\left(\tau^{2} j_{k} j_{l}\right)}{\left(\tau^{2}\left(j_{k}^{2}+j_{k} j_{l}+j_{l}^{2}\right)+3\right)\left(\tau^{2}\left(j_{k}^{2}-j_{k} j_{l}+j_{l}^{2}\right)+3\right)}, & k \neq l, \\ 1, & k=l .\end{cases}
$$

Thus we see that

$$
\operatorname{det}\left(M^{-1} \mathscr{B}\right)=\frac{F(\tau)}{G(\tau)},
$$

where $F(\tau)$ and $G(\tau)$ are polynomials of integral coefficients in $\tau$ and they have no common factor, i.e. $(F, G)=1$. Observe that $\operatorname{det}\left(M^{-1} \mathscr{B}\right)=1$ when $\tau=0$. In view of the assumption that $\tau$ is transcendental, we have that $\operatorname{det}\left(M^{-1} \mathscr{B}\right) \neq 0$. So $\operatorname{det}(\mathscr{B}) \neq 0$. Take $\bar{\Pi}=\left[0, \varepsilon_{0}^{1 / 2}\right]^{N}$. Then Measure $\bar{\Pi}=\varepsilon_{0}^{N / 2}$ and Diameter $\bar{\Pi}=\varepsilon_{0}^{1 / 2}$. Define $\Phi: \zeta \mapsto \xi$ by

$$
\lambda^{(N)}+\mathscr{B} \zeta=\xi .
$$

And let $\Pi=\Phi(\bar{\Pi})$. Thus,

$$
\frac{1}{C} \varepsilon_{0}^{N / 4} \leq \text { Measure } \Pi \leq C \varepsilon_{0}^{N / 4}, \quad \frac{1}{C} \varepsilon_{0} \leq \text { Diameter } \Pi \leq C \varepsilon_{0},
$$

and

$$
\omega^{0}(\zeta)=\xi, \quad \Omega^{0}(\xi)=\lambda^{\infty}-S \mathscr{B}^{-1} \lambda^{(N)}+S \mathscr{B}^{-1} \xi .
$$

Observe that $\left|S_{k l}\right| \leq C /|k|^{2}$ for $k \in \overline{\mathbb{Z}} \backslash J, l \in J$. It follows that the Assumptions A and (1.7) and (1.8) of Assumption $\mathbf{B}$ are fulfilled with taking $\kappa=1$. Arbitrarily take an infinite dimensional integer vector $l=\left(l_{j} \in \mathbb{Z}: j \in \overline{\mathbb{Z}} \backslash J\right)$ with $1 \leq|l| \leq 2$ (here $\left.|l|=\sum_{j \in \overline{\mathbb{Z}} \backslash J}\left|l_{j}\right|\right)$. Observe that where $0 \neq k \in \mathbb{Z}^{N}$, the function $(k, \xi)+\left\langle l, \Omega^{0}(\xi)\right\rangle$ is a affine function of $\xi$. Thus, letting $\xi_{0}$ is the direction such that $\frac{d}{d \xi_{0}}(k, \xi)=|k|$ along the direction, then

$$
\frac{d}{d \xi_{0}}\left((k, \xi)+\left\langle l, \Omega^{0}(\xi)\right\rangle\right)=\left(k, \xi_{0}\right)+\left\langle l, \Omega^{0}\left(\xi_{0}\right)\right\rangle
$$

And note that the affine function can be written as $F(\tau) / G(\tau)$ where $F, G$ are polynomials of integral coefficient in $\tau$. And note that when $\tau=0$

$$
\left(k, \xi_{0}\right)+\left\langle l, \Omega^{0}\left(\xi_{0}\right)\right\rangle=\left(k, \xi_{0}\right)=|k| \neq 0 .
$$

Thus

$$
(*):=\frac{d}{d \xi_{0}}\left((k, \xi)+\left\langle l, \Omega^{0}(\xi)\right\rangle\right)=\left(k, \xi_{0}\right)+\left\langle l, \Omega^{0}\left(\xi_{0}\right)\right\rangle \neq 0 .
$$

From (9.37) and the fact $\left|S_{k l}\right| \leq C /|k|^{2}$ for any $k \in \overline{\mathbb{Z}} \backslash J, l \in J$, it follows that there are a constant $C_{0}>0$ and an integer $j_{0} \in \overline{\mathbb{Z}} \backslash J$ such that

$$
\left|\left\langle l, \Omega^{0}\left(\xi_{0}\right)\right\rangle\right| \leq C_{0} /\left|j_{0}\right| .
$$


We can choose $J$ such that $\left|j_{0}\right|$ large enough with $C_{0} /\left|j_{0}\right|<1$. Thus there is $c_{0}>$ such that $(*)>c_{0}>0$. This verifies (1.26). Let

$$
D_{p}:=D_{p}\left(\varepsilon_{0}\right)=\left\{(x, y, \hat{z}, \overline{\bar{z}}) \in \mathbb{C}^{N} /(2 \pi \mathbb{Z})^{N} \times \mathbb{Z}^{N} \times h_{p} \times h_{p}:|\mathfrak{I} x| \leq s_{0},|y| \leq \varepsilon_{0},\|\mid \hat{z}\|_{p} \leq \varepsilon_{0}^{1 / 3},\|\overline{\hat{z}}\|_{p} \leq \varepsilon_{0}^{1 / 3}\right\} .
$$

By (9.31), we verify Assumption $\mathbf{C}$ and

$$
\|\left.\left\lfloor X_{\hat{G}+\tilde{R}}\right\rceil\right|_{q, D_{p} \times \Pi} \leq C \varepsilon_{0},\left|\left\lfloor\partial_{\xi} X_{\hat{G}+\tilde{R}}\right\rceil\right|_{q, D_{p} \times \Pi} \leq C \sqrt{\varepsilon_{0}} .
$$

In (9.4), $u$ is real if $z_{-j}$ is the complex conjugate of $z_{j}$. It follows that Assumption $\mathbf{D}$ holds true. Finally, Assumption E holds true clearly, since $B=0$.

By Theorem 1.1 we have the following theorem.

Theorem 9.5. Assume $\frac{2 \pi}{T}$ is transcendental. Around the neighborhood of $u=0$, BBM equation (9.40) has many (the initial value set of $N$-dimensional positive Lebesgue measure) smooth solutions which are quasi-periodic in time, linear stable and of zero Lyapunov exponent. More exactly, there exists $\varepsilon_{0}^{*}=\varepsilon_{0}^{*}(N, \tau, J)>0$ depending on $N, \tau, J$ such that for any $0<\varepsilon_{0}<\varepsilon_{0}^{*}$ there is a subset $\breve{\Pi}$ of the initial value set $\Pi_{0}:=\left[\sqrt{\varepsilon_{0}}, 2 \sqrt{\varepsilon_{0}}\right]^{N}$ with

$$
\operatorname{Leb} \breve{\Pi}=\left(\operatorname{Leb} \Pi_{0}\right)\left(1-C \frac{1}{\left|\log \varepsilon_{0}\right|}\right)
$$

and for any $\xi=\left(\xi_{l}: l=1, \ldots, N\right) \in \breve{\Pi}$, BBM equation has a quasi-periodic solution $u(t, x)$ of frequency $\omega \in \mathbb{R}^{N}$ in time $t$

$$
u(t, x)=\sum_{k \in \mathbb{Z}^{N}, j \in \mathbb{Z} \backslash\{0\}} \hat{u}(k, j) e^{\mathbf{i}(k, \omega)} e^{\mathbf{i} j \tau x}
$$

satisfying

$$
\begin{gathered}
\left|\omega-\omega_{0}\right| \leq C \sqrt{\varepsilon_{0}}, \omega \in \mathbb{R}^{N}, \omega_{0}=\left(\frac{\tau j_{l}}{1+\tau^{2} j_{l}^{2}}: j_{t} \in J\right) \in \mathbb{R}^{N}, \\
\left|\hat{u}\left(e_{l}, j_{l}\right)-\xi_{l}\right|<C \varepsilon_{0}^{1 / 3}, e_{l}-l^{\text {th }} \text { unit vector of } \mathbb{Z}^{N}, j_{l} \in J, l=1, \ldots, N,
\end{gathered}
$$

and

$$
\sum_{(k, j) \notin \mathscr{S}} \mid \hat{u}\left(k,\left.j\right|^{2} e^{|k| s_{0}+2 a|j|}|j|^{2 p}<C \varepsilon_{0}^{1 / 3}, \quad \mathscr{S}=\left(e_{l}, j_{l}\right): l=1, \ldots, N,\right.
$$

where some constants $s_{0}>0, a>0$ and $p>1 / 2$.

Remark 11. Theorem [.1] applies to more general BBM equation:

$$
u_{t}-u_{x x t}+u_{x}+F(u) u_{x}=0, \quad u(t, 0)=u(t, T),
$$

and Hirota-Satsuma equation

$$
u_{t}-u_{x x t}+u_{x}-F(u) u_{t}-u_{x} \partial_{x}^{-1} u_{t}=0, \quad u(t, 0)=u(t, T),
$$

where $F(u)=u+\sum_{j \geq 2} c_{j} u^{j}$ is an analytic function of $u$ with $c_{j} \in \mathbb{R}$.

Remark 12. We are glad to mention a recent paper [34], where the linear stability of traveling wave solution of $B B M$ is studied among the other things. 


\section{Application to $d$-dimensional generalized Pochhammer-Chree equa- tion}

The Pochhammer-Chree (gPC) equation represents a nonlinear model of longitudinal wave propagation of elastic rods [18, 40]. See also [10, 17, 38, 39], for example. Consider a $d$-dimensional generalized Pochhammer-Chree (gPC) equation

$$
\left\{\begin{array}{l}
u_{t t}-\Delta u-\Delta u_{t t}+\Delta\left(u^{3}\right)=0, x \in \Omega \subset \mathbb{R}^{d}, \\
\left.u\right|_{\partial \Omega}=0,
\end{array}\right.
$$

where $\Omega=\Pi_{j=1}^{d}\left[0, T_{j}\right]$ with $T_{j}>0$.

Let

$$
\phi_{k}(x)=\sin k_{1} \tau_{1} x_{1} \cdots \sin k_{d} \tau_{d} x_{d}, k \in \mathbb{Z}_{+}^{d}, \tau_{j}=\frac{2 \pi}{T_{j}}
$$

with $\mathbb{Z}_{+}^{d}=\left\{k=\left(k_{1}, \cdots, k_{d}\right) \mid k_{j} \in \mathbb{Z}_{+}, j=1, \cdots, d\right\}$, where $\mathbb{Z}_{+}=\{1,2,3, \cdots\}$ is the set of all positive integers.

Let

$$
u(t, x)=\sum_{k \in \mathbb{Z}_{+}^{d}} u_{k}(t) \phi_{k}(x)
$$

and put 10.3 into 10.1). Then we have

$$
\ddot{u}_{k}+\|k\|^{2} u_{k}+\|k\|^{2} \ddot{u}_{k}+\|k\|^{2} \widetilde{G}_{k}(u)=0, k \in \mathbb{Z}_{+}^{d},
$$

where

$$
\begin{gathered}
\|k\|^{2}=\tau_{1}^{2} k_{1}^{2}+\cdots+\tau_{d}^{2} k_{d}^{2}, k=\left(k_{1}, \cdots, k_{d}\right), \\
\widetilde{G}_{k}(u)=\sum_{m, n, l \in \mathbb{Z}_{+}^{d}} \widetilde{C}_{m n l k} u_{m} u_{n} u_{l},
\end{gathered}
$$

and

$$
\widetilde{C}_{m n l k}=\int_{\Omega} \phi_{m} \phi_{n} \phi_{l} \phi_{k} d x, \quad m, n, l, k \in \mathbb{Z}_{+}^{d}
$$

Rewrite 10.4 as

$$
\ddot{u}_{k}+\lambda_{k} u_{k}+\lambda_{k} \widetilde{G}_{k}(u)=0, \quad k \in \mathbb{Z}_{+}^{d}, \quad \lambda_{k}=\frac{\|k\|^{2}}{1+\|k\|^{2}}
$$

Let

$$
u_{k}=\frac{1}{\sqrt[4]{\lambda_{k}}} w_{k}, \quad \dot{u}_{k}=\sqrt[4]{\lambda_{k}} v_{k}, \quad k \in \mathbb{Z}_{+}^{d}
$$

Then 10.8 reads

$$
\left\{\begin{array}{l}
\dot{w}_{k}=\sqrt{\lambda_{k}} v_{k} \\
\dot{v}_{k}=-\sqrt{\lambda_{k}} w_{k}-G_{k}(w),
\end{array}\right.
$$

where

$$
\begin{aligned}
G_{k}(w) & =\frac{\lambda_{k}}{\sqrt[4]{\lambda_{k}}} \widetilde{G}_{k}(u)=\frac{\lambda_{k}}{\sqrt[4]{\lambda_{k}}} \sum_{m, n, l \in \mathbb{Z}_{+}^{d}} \widetilde{C}_{m n l k} \frac{w_{m} w_{n} w_{l}}{\sqrt[4]{\lambda_{m}} \sqrt[4]{\lambda_{n}} \sqrt[4]{\lambda_{l}}} \\
& =\lambda_{k} \sum_{m, n, l \in \mathbb{Z}_{+}^{d}} C_{m n l k}^{*} w_{m} w_{n} w_{l}, \\
C_{m n l k}^{*} & =\frac{1}{\sqrt[4]{\lambda_{m}} \sqrt[4]{\lambda_{n}} \sqrt[4]{\lambda_{l}} \sqrt[4]{\lambda_{k}}} \int_{\Omega} \phi_{m} \phi_{n} \phi_{l} \phi_{k} d x .
\end{aligned}
$$


We write $G_{k}(w)$ in the form of gradient:

$$
\begin{aligned}
G_{k}(w)= & \lambda_{k} \sum_{m, n, l} C_{m n l k}^{*} w_{m} w_{n} w_{l} \\
= & \frac{1}{4}\left(\lambda_{m} \sum_{n, l, k} C_{m n l k}^{*} w_{n} w_{l} w_{k}+\lambda_{n} \sum_{m, l, k} C_{m n l k}^{*} w_{m} w_{l} w_{k}\right. \\
& \left.+\lambda_{l} \sum_{m, n, k} C_{m n l k}^{*} w_{m} w_{n} w_{k}+\lambda_{k} \sum_{m, n, l} C_{m n l k}^{*} w_{m} w_{n} w_{l}\right) \\
= & \partial_{w_{k}} G(w),
\end{aligned}
$$

where

$$
\begin{gathered}
G(w)=\sum_{m, n, l, k} C_{m n l k} w_{m} w_{n} w_{l} w_{k}, \\
C_{m n l k}=\frac{\lambda_{m}+\lambda_{n}+\lambda_{l}+\lambda_{k}}{4 \sqrt[4]{\lambda_{m} \lambda_{n} \lambda_{l} \lambda_{k}}} \int_{\Omega} \phi_{m} \phi_{n} \phi_{l} \phi_{k} d x .
\end{gathered}
$$

By (10.2), we have

$$
C_{m n l k}=0, \quad \text { unless there is a combination of }+ \text { and }- \text { such that } m \pm n \pm l \pm k=0 .
$$

Now (10.9) can be written as a Hamiltonian system

$$
\left\{\begin{array}{l}
\dot{w}_{k}=\frac{\partial H}{\partial v_{k}}, \\
\dot{v}_{k}=-\frac{\partial H}{\partial w_{k}}, k \in \mathbb{Z}_{+}^{d},
\end{array}\right.
$$

where

$$
\begin{aligned}
& H=\sum_{k \in \mathbb{Z}_{+}^{d}} \frac{1}{2} \sqrt{\lambda_{k}}\left(w_{k}^{2}+v_{k}^{2}\right)+G(w), \\
& G(w)=\sum_{m \pm n \pm l \pm k=0} C_{m n l k} w_{m} w_{n} w_{l} w_{k} .
\end{aligned}
$$

Lemma 10.1. Let $\partial_{w} G=\left(\partial_{w_{k}} G: k \in \mathbb{Z}_{+}^{d}\right)$. Then

$$
\left\|\left\lfloor\partial_{w} G\right\rceil\right\|_{p} \leq c\|w\|_{p}^{3} .
$$

Proof. Recall (10.14) and (10.16). Then

$$
\partial_{w_{k}} G=G_{k}(w)=\lambda_{k} \sum_{ \pm m \pm n \pm l=k} C_{m n l k} w_{m} w_{n} w_{l} .
$$

Thus

$$
\left\lfloor\partial_{w_{k}} G\right\rceil \leq C \sum_{ \pm m \pm n \pm l=k}\left|w_{m}\right|\left|w_{n}\right|\left|w_{l}\right|=C(w * w * w)(k)
$$

where $*$ is the convolution in $\ell_{2}\left(\mathbb{Z}_{+}^{d}\right)$. So

$$
\left\|\left\lfloor\partial_{w} G\right\rceil\right\|_{p} \leq C\|w\|_{p}^{3}
$$


Let

$$
z_{k}=\frac{1}{\sqrt{2}}\left(w_{k}+\sqrt{-1} v_{k}\right), \bar{z}_{k}=\frac{1}{\sqrt{2}}\left(w_{k}-\sqrt{-1} v_{k}\right), k \in \mathbb{Z}_{+}^{d} .
$$

This is a symplectic transformation, which changes 10.18 into

$$
H=\sum_{k} \sqrt{\lambda_{k}} z_{k} \bar{z}_{k}+G(z, \bar{z})
$$

where the symplectic structure is $\sqrt{-1} d \bar{z} \wedge d z$, and

$$
G(z, \bar{z})=G(w)=\sum_{m \pm n \pm l \pm k=0} \frac{1}{4} C_{m n l k}\left(z_{m}+\bar{z}_{m}\right)\left(z_{n}+\bar{z}_{n}\right)\left(z_{l}+\bar{z}_{l}\right)\left(z_{k}+\bar{z}_{k}\right) .
$$

A polynomial of degree $n$ of $d$-dimension variable $x=\left(x_{1}, \ldots, x_{d}\right)$ can be written as

$$
P(x)=\sum_{\alpha \in \mathbb{N}^{d},|\alpha| \leq n} C_{\alpha} x^{\alpha}, \quad x^{\alpha}=x_{1}^{\alpha_{1}} \cdots x_{d}^{\alpha_{d}}, \alpha=\left(\alpha_{1}, \ldots, \alpha_{d}\right)
$$

where $C_{\alpha}$ 's are coefficients and there is $\alpha$ with $|\alpha|=n$ such that $C_{\alpha} \neq 0$. We call that $P(x)$ is a polynomial with coefficients in the field of the rational numbers, if all coefficients $C_{\alpha}$ 's are in $\mathbb{Q}$. By $\mathbb{Q}_{n}[x]$ denote the set of all polynomials of degree $n$ with coefficients in the field of the rational numbers. Let $\mathbb{Q}[x]=\bigcup_{n \in \mathbb{N}} \mathbb{Q}_{n}[x]$ where $\mathbb{N}=\{1,2, \ldots\}$. Clearly, the set $\mathbb{Q}[x]$ is countable. For any $P \in \mathbb{Q}[x]$, let $S_{P}$ be the set of all solutions to the polynomial equation $P(x)=0$. Let $\Theta=[1,2]^{d}$. We claim that the Lebesgue measure of $S_{P} \cap \Theta$ is zero. In fact, the result is clear when the dimension $d=1$. The proof for $d \geq 2$ can be finished by Fubini Theorem and mathematical induction. Let

$$
S=\bigcup_{P \in \mathbb{Q}[x]}\left(S_{P} \bigcap \Theta\right) .
$$

Considering that $\mathbb{Q}[x]$ is countable, we have that Leb $S=0$. Define $\tilde{\Theta}=\Theta \backslash S$. When $\tau \in \tilde{\Theta}$, we call $\tau$ is typical. At this time, $\operatorname{Leb} \tilde{\Theta}=1$. Therefore, for any $P \in \mathbb{Q}[x]$ and any $\tau \in \tilde{\Theta}$, we have $P(\tau) \neq 0$.

Fix arbitrarily integer $N$ which denotes the number of the incited oscillators. Let

$$
J=\left\{j_{1}, \cdots, j_{N}|L<| j_{1}|<| j_{2}|<\cdots<| j_{N} \mid, j_{t} \in \mathbb{Z}_{+}^{d}, t=1,2, \cdots, N\right\},
$$

where $L$ is supposed to be large $L \gg 1$ and $L \gg N$. The large $L \gg 1$ means that the incited oscillators are of high frequency. Here it should be pointed out that the assumption $L \gg 1$ is just for simplifying the following computation. Split $z=\left(z_{j}: j \in \mathbb{Z}_{+}^{d}\right)=(\tilde{z}, \hat{z})$ with

$$
\tilde{z}=\left(z_{j_{1}}, \cdots, z_{j_{N}}\right), \hat{z}=z \ominus \tilde{z} .
$$

We will eliminate those terms of lower frequencies in $G(z, \bar{z})$, which involve $\tilde{z}$, as many as possible by Birkhoff normal form. To this end we need the following lemma.

Lemma 10.2. Assume that $\tau=\left(\tau_{1}, \cdots, \tau_{d}\right)$ is typical, i.e., $\tau \in \tilde{\Theta}$.

(1) If $m \pm n \pm k \pm l=0$ and $\{m, l\} \neq\{n, k\}$, then

$$
\sqrt{\lambda_{m}}-\sqrt{\lambda_{n}}+\sqrt{\lambda_{l}}-\sqrt{\lambda_{k}} \neq 0
$$

(2) If $m \pm n \pm k \pm l=0$, then

$$
\pm\left(\sqrt{\lambda_{m}}+\sqrt{\lambda_{n}}+\sqrt{\lambda_{l}}+\sqrt{\lambda_{k}}\right) \neq 0, \pm\left(\sqrt{\lambda_{m}}+\sqrt{\lambda_{n}}+\sqrt{\lambda_{l}}-\sqrt{\lambda_{k}}\right) \neq 0 ;
$$


(4)

$$
\sqrt{\lambda_{m}} \pm \sqrt{\lambda_{n}} \pm \sqrt{\lambda_{l}}+p \neq 0, \quad p=0, \pm 1, \cdots
$$

$$
\sqrt{\lambda_{m}} \pm \sqrt{\lambda_{n}}+p \neq 0, p=0, \pm 1, \pm 2,
$$

where $m \neq n$ for $\sqrt{\lambda_{m}}-\sqrt{\lambda_{n}}+p$.

Proof. We give the proof for only case (1). The remaining is similar. Recall that $\lambda_{m}$ is a function of $\tau$ and

$$
\lambda_{m}=\lambda_{m}(\tau)=\frac{\|m\|^{2}}{1+\|m\|^{2}}=\frac{\tau_{1}^{2} m_{1}^{2}+\cdots+\tau_{d}^{2} m_{d}^{2}}{1+\tau_{1}^{2} m_{1}^{2}+\cdots+\tau_{d}^{2} m_{d}^{2}}, \forall m=\left(m_{1}, \ldots, m_{d}\right) \in \mathbb{Z}_{+}^{d} .
$$

Write $m=\left(m_{1}, \ldots, m_{d}\right) \in \mathbb{Z}_{+}^{d}$, etc. Since $\{m, l\} \neq\{n, k\}$, we can assume that $m_{1} \neq n_{1}$ or $l_{1} \neq k_{1}$ without loss of generality. For $\tau=\left(\tau_{1}, \tau_{2}, \ldots, \tau_{d}\right) \in \mathbb{R}^{d}$, let $\tau^{(1)}=\left(\tau_{1}, 0, \ldots, 0\right)$. Then

$$
\lambda_{m}\left(\tau^{(1)}\right)=\frac{\tau_{1}^{2} m_{1}^{2}}{1+\tau_{1}^{2} m_{1}^{2}}=\frac{m_{1}^{2}}{\tau_{1}^{-2}+m_{1}^{2}}=\frac{m_{1}^{2}}{s+m_{1}^{2}}:=\lambda_{m}(s), \quad \text { here } s=\tau_{1}^{-2}
$$

Set

$$
\Gamma(s)=\sqrt{\lambda_{m}(s)}-\sqrt{\lambda_{n}(s)}+\sqrt{\lambda_{l}(s)}-\sqrt{\lambda_{k}(s)}
$$

Then by a simple computation we have

$$
-\left.2 \frac{d \Gamma}{d s}\right|_{s=0}=\frac{1}{m_{1}^{2}}-\frac{1}{n_{1}^{2}}+\frac{1}{l_{1}^{2}}-\frac{1}{k_{1}^{2}}:=(*)
$$

and

$$
\left.\frac{4}{3} \frac{d^{2} \Gamma}{d s^{2}}\right|_{s=0}=\frac{1}{m_{1}^{4}}-\frac{1}{n_{1}^{4}}+\frac{1}{l_{1}^{4}}-\frac{1}{k_{1}^{4}}:=(* *) .
$$

By $m_{1} \pm n_{1} \pm k_{1} \pm l_{1}=0$ and $m_{1} \neq n_{1}$ or $l_{1} \neq k_{1}$, we get that either $(*) \neq 0$ or $(* *) \neq 0$. It follows that there exists a $s_{0} \in \mathbb{R}$ such that $\Gamma\left(s_{0}\right) \neq 0$. Moreover, there exists a $\tau_{0} \in \mathbb{R}^{d}$ such that

$$
\gamma\left(\tau_{0}\right):=\sqrt{\lambda_{m}\left(\tau_{0}\right)}-\sqrt{\lambda_{n}\left(\tau_{0}\right)}+\sqrt{\lambda_{l}\left(\tau_{0}\right)}-\sqrt{\lambda_{k}\left(\tau_{0}\right)} \neq 0
$$

If $\sqrt{\lambda_{m}}-\sqrt{\lambda_{n}}+\sqrt{\lambda_{l}}-\sqrt{\lambda_{k}}=0$ where $\lambda_{m}=\lambda_{m}(\tau)$, etc., then $\sqrt{\lambda_{m}}+\sqrt{\lambda_{l}}=\sqrt{\lambda_{n}}+\sqrt{\lambda_{k}}$. It follows

$$
\left[\left(\lambda_{m}+\lambda_{l}-\lambda_{n}-\lambda_{k}\right)^{2}-4\left(\lambda_{n} \lambda_{k}+\lambda_{m} \lambda_{l}\right)\right]^{2}-64 \lambda_{m} \lambda_{n} \lambda_{k} \lambda_{l}=0
$$

Multiplying (10.22) by $\left(1+\|m\|^{2}\right)^{4}\left(1+\|n\|^{2}\right)^{4}\left(1+\|k\|^{2}\right)^{4}\left(1+\|l\|^{2}\right)^{4}$, and noting $m \pm n \pm k \pm l=0$, we get

$$
P(\tau):=\sum_{\substack{10 \leq|\alpha| \leq 26 \\ \alpha \in \mathbb{Z}_{+}^{d}}} C_{\alpha} \tau^{\alpha}=0
$$

where $C_{\alpha} \in \mathbb{Z}$. By $\gamma\left(\tau_{0}\right) \neq 0$, we get that there exists a coefficient $C_{\alpha} \neq 0$. Thus, $P(x) \in \mathbb{Q}[x]$. Thus $P(\tau) \neq 0$ when $\tau \in \tilde{\Theta}$, which is contradictory to 10.22 . This completes the proof.

Lemma 10.3. Assume $\tau \in \tilde{\Theta}$. If $m \pm n \pm k \pm l=0$, and $\{m, n, k, l\} \cap J \neq \emptyset$. Let $\triangle=\sqrt{\lambda_{m}} \pm \sqrt{\lambda_{n}} \pm \sqrt{\lambda_{k}} \pm \sqrt{\lambda_{l}}$ (excluding $\Delta=\sqrt{\lambda_{m}}-\sqrt{\lambda_{n}}+\sqrt{\lambda_{l}}-\sqrt{\lambda_{k}}$ with $\{m, l\}=\{n, k\}$ ). Then there exists a constant $C>0$ such that $|\triangle|>C>0$. 
Proof. We give proof for only the most difficult case

$$
\triangle=\sqrt{\lambda_{m}}-\sqrt{\lambda_{n}}+\sqrt{\lambda_{l}}-\sqrt{\lambda_{k}}
$$

where there is a combination of + and - such that $m \pm n \pm l \pm k=0$, and $\{m, l\} \neq\{n, k\}$, and assuming

$$
m, n \in J .
$$

Let $\widetilde{N}>0$ be a large number which is to be specified later. If $|l| \leq \widetilde{N}$ (or $|k| \leq \widetilde{N}$ ), by $m \pm n \pm k \pm l=0$ and $m, n \in J$, we have that there exists $C_{1}=C_{1}(\widetilde{N})>0$ such that $|k| \leq C_{1}(\widetilde{N})$. Recall that $m, n \in J$ implies $|m|+|n| \leq 2\left(L+j_{N}\right)$. So by taking $\tilde{N}>L+j_{N}$ and using Lemma 10.2 there is a constant $C_{2}=C_{2}(\widetilde{N})>0$ such that

$$
|\Delta| \geq C_{2}(\widetilde{N})>0 .
$$

Now we assume $|l|>\widetilde{N}$ and $|k|>\widetilde{N}$. Then

$$
\begin{aligned}
|\Delta| & =\left|\sqrt{\lambda_{m}}-\sqrt{\lambda_{n}}+\sqrt{\lambda_{l}}-\sqrt{\lambda_{k}}\right| \\
& =\left|\sqrt{\lambda_{m}}-\sqrt{\lambda_{n}}+\sqrt{1-\frac{1}{1+\|l\|^{2}}}-\sqrt{1-\frac{1}{1+\|k\|^{2}}}\right| \\
& \geq\left|\sqrt{\lambda_{m}}-\sqrt{\lambda_{n}}\right|-\frac{C_{0}}{\widetilde{N}^{2}},
\end{aligned}
$$

where $C_{0}=C_{0}(\Theta)>0$ is a constant. Since $m \neq n$, and $m, n \in J$, we have that there exists a constant $C_{3}=C_{3}\left(L+j_{N}\right)$ such that

$$
\left|\sqrt{\lambda_{m}}-\sqrt{\lambda_{n}}\right| \geq C_{3}\left(L+j_{N}\right)
$$

Choose $\widetilde{N}$ large enough such that

$$
C_{3}-\frac{C_{0}}{\widetilde{N}^{2}} \geq \frac{C_{3}}{2}
$$

Then by 10.23,

$$
|\Delta| \geq \frac{C_{3}}{2}
$$

Consequently, let $C_{4}=C_{4}(N, \widetilde{N})=\min \left\{C_{2}(\widetilde{N}), \frac{1}{2} C_{3}(N)\right\}$. Then $|\Delta| \geq C_{4}(L, N, \widetilde{N})>0$.

Lemma 10.4. Assume $\tau \in \tilde{\Theta}$. There exists a symplectic $\Phi=I d .+O\left(\|z\|_{p}\right)$ such that

$$
H \circ \Phi=\sum_{k \in \mathbb{Z}_{+}^{d}} \sqrt{\lambda_{k}} z_{k} \bar{z}_{k}+\bar{G}+\widehat{G}+\breve{G}
$$

where

$$
\begin{aligned}
& \bar{G}=\sum_{\substack{k, l \in \mathbb{Z}_{+}^{d} \\
\{k, l\} \cap J \neq \emptyset}} \bar{G}_{k l}\left|z_{k}\right|^{2}\left|z_{l}\right|^{2}, \\
& \bar{G}_{k l}=\left\{\begin{array}{l}
\left(\frac{3}{8}\right)^{d} T_{1} \cdots T_{d} \sqrt{\lambda_{k}}, k=l, \\
\frac{1}{2} \sum_{1 \leq p \leq d}\left(\begin{array}{c}
d \\
p
\end{array}\right)\left(\frac{1}{4}\right)^{p}\left(\frac{3}{8}\right)^{q} T_{1} \cdots T_{d}\left(\sqrt{\lambda_{k}}+\sqrt{\lambda_{l}}\right), k \neq l,
\end{array}\right. \\
& \left\|X_{\widehat{G}}\right\|_{p} \leq C\|\hat{z}\|_{p}^{3},\left\|X_{\breve{G}}\right\|_{p} \leq C\|z\|_{p}^{5} .
\end{aligned}
$$


Proof. Decompose $G(z, \bar{z})$ in $(10.21$ as follows

$$
\begin{aligned}
G(z, \bar{z}) & =G^{(1)}(z, \bar{z})+G^{(2)}(z, \bar{z})+G^{(3)}(z, \bar{z}), \\
G^{(1)}(z, \bar{z}) & =\sum_{\substack{\{m, k\} \cap J \neq \emptyset \\
G^{(2)}(z, \bar{z})}} C_{m m k k} z_{m} \bar{z}_{m} z_{k} \bar{z}_{k}, \\
G^{(3)}(z, \bar{z}) & \sum_{\substack{m \pm n \pm l \pm k=0 \\
\{m, n, l, k\} \cap J \neq \emptyset}} \frac{1}{4} C_{m n l k}\left(z_{m}+\bar{z}_{m}\right)\left(z_{n}+\bar{z}_{n}\right)\left(z_{l}+\bar{z}_{l}\right)\left(z_{k}+\bar{z}_{k}\right)-G^{(1)}(z, \bar{z}), \\
& \sum_{\substack{m \pm n \pm l \pm k=0 \\
\{m, n, l, k\} \cap J=\emptyset}} \frac{1}{4} C_{m n l k}\left(z_{m}+\bar{z}_{m}\right)\left(z_{n}+\bar{z}_{n}\right)\left(z_{l}+\bar{z}_{l}\right)\left(z_{k}+\bar{z}_{k}\right) .
\end{aligned}
$$

By (10.15), then

$$
\bar{G}_{k l}:=C_{k k l l}=\frac{1}{2} \frac{\lambda_{k}+\lambda_{l}}{\sqrt{\lambda_{k} \lambda_{l}}} \int_{\Omega} \phi_{k}^{2} \phi_{l}^{2} d x
$$

By (10.2), we have

$$
\begin{aligned}
\bar{G}_{k l}= & \frac{1}{2}\left(\sqrt{\lambda_{k} / \lambda_{l}}+\sqrt{\lambda_{l} / \lambda_{k}}\right) \prod_{j=1}^{d} \int_{0}^{T_{j}} \sin ^{2} k_{j} \tau_{j} x_{j} \sin ^{2} l_{j} \tau_{j} x_{j} d x_{j} \\
= & \left\{\begin{array} { c } 
{ ( \frac { 3 } { 8 } ) ^ { d } T _ { 1 } T _ { 2 } \cdots T _ { d } , \quad k = l , } \\
{ \frac { 1 } { 2 } \sum \begin{array} { c } 
{ d } \\
{ p + q = d } \\
{ p \geq 1 }
\end{array} }
\end{array} \left(\begin{array}{c}
\left.\frac{1}{4}\right)^{p}\left(\frac{3}{8}\right)^{q} T_{1} \cdots T_{d}\left(\sqrt{\lambda_{k} / \lambda_{l}}+\sqrt{\lambda_{l} / \lambda_{k}}\right), k \neq l .
\end{array}\right.\right.
\end{aligned}
$$

Rewrite

$$
\begin{aligned}
G^{(2)}(z, \bar{z})= & \sum \frac{1}{4} C_{m n l k} z_{m} z_{n} z_{l} z_{k}+\sum \frac{1}{4} C_{m n l k} z_{m} z_{n} z_{l} \bar{z}_{k}+\sum_{\{m, n\} \neq\{k, l\}} \frac{1}{4} C_{m n l k} z_{m} z_{n} \bar{z}_{l} \bar{z}_{k} \\
& +\sum \frac{1}{4} C_{m n l k} z_{m} \bar{z}_{n} \bar{z}_{l} \bar{z}_{k}+\sum \frac{1}{4} C_{m n l k} \bar{z}_{m} \bar{z}_{n} \bar{z}_{l} \bar{z}_{k}
\end{aligned}
$$

where the sum runs over $m, n, k, l \in \mathbb{Z}_{+}^{d}$ with some $m \pm n \pm k \pm l=0$. Let $F=F(z, \bar{z})$ be of the same form as $G^{(2)}(z, \bar{z})$ :

$$
\begin{aligned}
F=F(z, \bar{z})= & \sum \frac{C_{m n l k} z_{m} z_{n} z_{k} z_{l}}{4 \mathbf{i}\left(\sqrt{\lambda_{m}}+\sqrt{\lambda_{n}}+\sqrt{\lambda_{l}}+\sqrt{\lambda_{k}}\right)}+\sum \frac{C_{m n l k} z_{m} z_{n} z_{k} \bar{z}_{l}}{4 \mathbf{i}\left(\sqrt{\lambda_{m}}+\sqrt{\lambda_{n}}+\sqrt{\lambda_{l}}-\sqrt{\lambda_{k}}\right)} \\
& +\sum \frac{C_{m n l k} z_{m} z_{n} \bar{z}_{k} \bar{z}_{l}}{4 \mathbf{i}\left(\sqrt{\lambda_{m}}+\sqrt{\lambda_{n}}-\sqrt{\lambda_{l}}-\sqrt{\lambda_{k}}\right)}+\sum \frac{C_{m n l k} z_{m} \bar{z}_{n} \bar{z}_{k} \bar{z}_{l}}{4 \mathbf{i}\left(\sqrt{\lambda_{m}}-\sqrt{\lambda_{n}}-\sqrt{\lambda_{l}}-\sqrt{\lambda_{k}}\right)} \\
& +\sum \frac{C_{m n l k} \bar{z}_{m} \bar{z}_{n} \bar{z}_{k} \bar{z}_{l}}{-4 \mathbf{i}\left(\sqrt{\lambda_{m}}+\sqrt{\lambda_{n}}+\sqrt{\lambda_{l}}+\sqrt{\lambda_{k}}\right)}
\end{aligned}
$$

where the sum runs over the same set as $G^{(2)}(z, \bar{z})$. By Lemma 10.3 it is easy to get

$$
\left\|\left\lfloor X_{F}\right\rceil\right\|_{p} \leq C\|z\|_{p}^{3} .
$$

Moreover, $\Psi:=\left.X_{F}^{t}\right|_{t=1}=I d .+O\left(\|z\|_{p}^{3}\right)$ maps a neighborhood of $z=0$ in $l_{p}$ into another neighborhood of $z=0$ in $l_{p}$, and

$$
H \circ \Psi=\sum_{k \in \mathbb{Z}_{+}^{d}} \sqrt{\lambda_{k}} z_{k} \bar{z}_{k}+\bar{G}+\widehat{G}+\breve{G}
$$


where $\bar{G}=G^{(1)}, \widehat{G}=G^{(3)}$,

$$
\breve{R}=\int_{0}^{1} \int_{0}^{t}\left\{\left\{\sum_{k \in \mathbb{Z}_{+}^{d}} \sqrt{\lambda_{k} z_{k} \bar{z}_{k}}, F\right\}, F\right\} \circ X_{F}^{\tau} d \tau d t+\int_{0}^{1}\{G, F\} \circ X_{F}^{t} d t .
$$

It is easy to check

$$
\left\|\left\lfloor X_{\widehat{G}}\right\rceil\right\|_{p} \leq C\|\hat{z}\|_{p}^{3}, \quad\left\|\left\lfloor X_{\breve{G}}\right\rceil\right\|_{p} \leq C\|z\|_{p}^{5}
$$

As in Section 9, restrict $|\tilde{z}|<C \varepsilon_{0}^{1 / 4}$ and $\|\hat{z}\|_{p} \leq C \varepsilon_{0}^{1 / 3}$. Then

$$
\left\|\left\lfloor X_{\widehat{G}+\breve{G}}\right\rceil\right\|_{p} \leq C\left(\|\hat{z}\|_{p}^{3}+\|z\|_{p}^{5}\right) \leq C \varepsilon_{0} .
$$

Introduce action-angle variables $(y, x)$ by

$$
z_{j_{k}}=\sqrt{\zeta_{k}+y_{k}} e^{-\mathbf{i} x_{k}}, \bar{z}_{j_{k}}=\sqrt{\zeta_{k}+y_{k}} e^{\mathbf{i} k_{k}}, k=1,2, \cdots, N, \quad j_{k} \in J
$$

where $\zeta=\left(\zeta_{1}, \ldots, \zeta_{n}\right) \in \mathbb{R}_{+}^{n}$ and

$$
\varepsilon_{0}^{1 / 2}<|\zeta| \leq 2 \varepsilon_{0}^{1 / 2}
$$

Then

$$
\sum_{j \in \mathbb{Z}_{+}^{d}} \sqrt{\lambda_{j}} z_{j} \bar{z}_{j}=\sum_{k=1}^{N} \sqrt{\lambda_{j_{k}}} \zeta_{k}+\sum_{k=1}^{N} \sqrt{\lambda_{j_{k}}} y_{k}+\sum_{j \in \mathbb{Z}_{+}^{d} \backslash J} \lambda_{j} z_{j} \bar{z}_{j}
$$

and

$$
\begin{aligned}
\bar{G}= & \sum_{1 \leq k, l \leq N} \bar{G}_{j_{k} l_{k}}\left(\zeta_{k}+y_{k}\right)\left(\zeta_{l}+y_{l}\right)+\sum_{1 \leq k \leq N, l \in \mathbb{Z}_{+}^{d} \backslash J} \bar{G}_{j_{k} l}\left(\zeta_{k}+y_{k}\right) z_{l} \bar{z}_{l} \\
& +\sum_{k \in \mathbb{Z}_{+}^{d} \backslash J, 1 \leq l \leq N} \bar{G}_{k j_{l}}\left(\zeta_{l}+y_{l}\right) z_{k} \bar{z}_{k} .
\end{aligned}
$$

Let

$$
\breve{G}(x, y, \hat{z}, \overline{\hat{z}} ; \zeta)=\breve{G}(\tilde{z}, \bar{z}, \hat{z}, \overline{\hat{z}})
$$

where $(\tilde{z}, \overline{\tilde{z}})$ are defined by (10.27). Let

$$
\omega^{0}(\zeta)=\lambda^{(N)}+T B \zeta, \Omega^{0}(\zeta)=\lambda^{\infty}+T S \zeta, \quad T=T_{1} \cdots T_{d},
$$

where

$$
\begin{aligned}
& \lambda^{(N)}=\left(\sqrt{\frac{\left\|j_{1}\right\|^{2}}{1+\left\|j_{1}\right\|^{2}}}, \cdots, \sqrt{\frac{\left\|j_{N}\right\|^{2}}{1+\left\|j_{N}\right\|^{2}}}\right), j_{1}, \cdots, j_{N} \in J, \\
& \lambda^{\infty}=\left\{\sqrt{\lambda_{j}}=\sqrt{\frac{\|j\|^{2}}{1+\|j\|^{2}}}: j \in \mathbb{Z}_{+}^{d} \backslash J\right\}, \\
& B=\left(B_{k l}: k, l \in J\right), \\
& B_{k l}=\left\{\begin{array}{l}
a, k=l, k \in J, \\
\frac{1}{2} b\left(\sqrt{\lambda_{k} / \lambda_{l}}+\sqrt{\lambda_{l} / \lambda_{k}}\right), k \neq l, k, l \in J, \\
a=\left(\frac{3}{8}\right)^{d}, b=\sum_{1 \leq p \leq d}\left(\begin{array}{c}
d \\
p
\end{array}\right)\left(\frac{1}{4}\right)^{p}\left(\frac{3}{8}\right)^{d-p}=\left(\frac{5}{8}\right)^{d}-a, \\
S=\left(S_{k l}: k \in \mathbb{Z}_{+}^{d} \backslash J, l \in J\right), \\
S_{k l}=\bar{G}_{k l}=\frac{1}{2} b\left(\sqrt{\lambda_{k}}+\sqrt{\lambda_{l}}\right), k \in \mathbb{Z}_{+}^{d} \backslash J, l \in J .
\end{array}\right.
\end{aligned}
$$


Then, up to a constant depending on $\zeta$, the Hamiltonian $H \circ \Phi$ in (10.24) can be written as

$$
H=H_{0}+R^{0}
$$

with

$$
\begin{gathered}
H_{0}=\sum_{j=1}^{N} \omega_{j}^{0}(\zeta) y_{j}+\sum_{j \in \mathbb{Z}_{+}^{d} \backslash J} \Omega_{j}^{0}(\zeta) z_{j} \bar{z}_{j}, \\
R^{0}=\sum_{1 \leq k, l \leq N} \bar{G}_{j_{k} l_{k}} y_{k} y_{l}+\sum_{k \in \mathbb{Z}_{+}^{d} \backslash J, 1 \leq l \leq N} \bar{G}_{k j_{l}} y_{l} z_{k} \bar{z}_{k}+\hat{G}(\hat{z}, \bar{z})+\breve{G}(\tilde{z}, \bar{z}, \hat{z}, \overline{\hat{z}} ; \zeta) .
\end{gathered}
$$

Let us write the matrix $B$ explicitly:

$$
B=\left(\begin{array}{cccc}
a & \frac{1}{2} b\left(\sqrt{\lambda_{j_{1}} / \lambda_{j_{2}}}+\sqrt{\lambda_{j_{2}} / \lambda_{j_{1}}}\right) & \cdots & \frac{1}{2} b\left(\sqrt{\lambda_{j_{1}} / \lambda_{j_{N}}}+\sqrt{\lambda_{j_{N}} / \lambda_{j_{1}}}\right) \\
\frac{1}{2} b\left(\sqrt{\lambda_{j_{2}} / \lambda_{j_{1}}}+\sqrt{\lambda_{j_{1}} / \lambda_{j_{2}}}\right) & a & \cdots & \frac{1}{2} b\left(\sqrt{\lambda_{j_{2}} / \lambda_{j_{N}}}+\sqrt{\lambda_{j_{N}} / \lambda_{j_{2}}}\right) \\
\vdots & \vdots & \ddots & \vdots \\
\frac{1}{2} b\left(\sqrt{\lambda_{j_{N}} / \lambda_{j_{1}}}+\sqrt{\lambda_{j_{1}} / \lambda_{j_{N}}}\right) & \frac{1}{2} b\left(\sqrt{\lambda_{j_{N}} / \lambda_{j_{2}}}+\sqrt{\lambda_{j_{2}} / \lambda_{j_{N}}}\right) & \cdots & a
\end{array}\right)
$$

where $j_{p} \in J, p=1,2, \cdots, N$. Recall $\sqrt{\lambda_{j}}=\sqrt{\frac{\|j\|^{2}}{1+\|j\|^{2}}}$. (Note $\|j\|=\sqrt{\tau_{1}^{2} j_{1}^{2}+\cdots+\tau_{d}^{2} j_{d}^{2}} \sim|j|$.) So

$$
\sqrt{\lambda_{j}}=\sqrt{1-\frac{1}{1+\|j\|^{2}}}=1+O\left(\frac{1}{L^{2}}\right), \quad j \in J
$$

It follows that

$$
B=\left(\begin{array}{cccc}
a & b & \cdots & b \\
b & a & \cdots & b \\
\vdots & \vdots & \ddots & \vdots \\
b & b & \cdots & a
\end{array}\right)+O\left(\frac{1}{L^{2}}\right)
$$

Then

$$
\operatorname{det} B=(a-b)^{N-1}(a+4 b)+O\left(\frac{N}{L^{2}}\right) \neq 0, \quad L \gg N
$$

and

$$
\begin{aligned}
B^{-1} & =\frac{1}{(a-b)(a+(N-1) b)}\left(\begin{array}{cccc}
a+(N-2) b & -b & \cdots & -b \\
-b & a+(N-2) b & \cdots & -b \\
\vdots & \vdots & \ddots & \vdots \\
-b & -b & \cdots & a+(N-2) b
\end{array}\right)+O\left(\frac{N}{L^{2}}\right) \\
& :=B_{0}^{-1}+O\left(\frac{N}{L^{2}}\right) .
\end{aligned}
$$

That is, $\left\|B^{-1}-B_{0}^{-1}\right\| \leq \frac{C}{L}$, where $C=C(N)$ depends on $N$. Take $\bar{\Pi}=\left[\sqrt{\varepsilon_{0}}, 2 \sqrt{\varepsilon_{0}}\right]^{N}$. Then Measure $\bar{\Pi}=\varepsilon_{0}^{N / 2}$ and Diameter $\bar{\Pi}=\varepsilon_{0}^{1 / 2}$. Define $\Phi: \zeta \mapsto \xi$ by

$$
T B \zeta=\xi
$$

And let $\Pi=\Phi(\bar{\Pi})$. Thus,

$$
\frac{1}{C} \varepsilon_{0}^{N / 4} \leq \text { Measure } \Pi \leq C \varepsilon_{0}^{N / 4}, \quad \frac{1}{C} \varepsilon_{0} \leq \text { Diameter } \Pi \leq C \varepsilon_{0},
$$


and

$$
\begin{gathered}
\omega^{0}(\xi)=\lambda^{(N)}+\xi, \\
\Omega^{0}(\xi)=\lambda^{\infty}+S B^{-1} \xi:=\left(\Omega_{j}^{0}(\xi): j \in \mathbb{Z}_{+}^{d} \backslash J\right), \\
H_{0}=\left(\omega^{0}(\xi), y\right)+\sum_{j \in \mathbb{Z}_{+}^{d} \backslash J} \Omega_{j}^{0}(\xi) z_{z} \bar{z}_{j} .
\end{gathered}
$$

By 10.37, Assumption A is obviously fulfilled. Recall

$$
S=\left(s_{i l}=\frac{b}{2}\left(\sqrt{\lambda_{i} / \lambda_{l}}+\sqrt{\lambda_{l} / \lambda_{i}}\right): i \in \mathbb{Z}_{+}^{d} \backslash J, l \in J\right),
$$

where $k$ denotes the row index of $S$ and $l$ the column index of $S$ and recall

$$
\lambda^{\infty}=\left(\sqrt{\lambda_{j}}=\sqrt{\frac{\|j\|^{2}}{1+\left.|| j\right|^{2}}}=1-O\left(\frac{1}{|j|^{2}}\right): j \in \mathbb{Z}_{+}^{d} \backslash J\right)
$$

Write $B^{-1}=\left(b_{k l}: k, l \in J\right)$. By (10.38), (10.40) and 10.41), we have

$$
\Omega_{j}^{0}=\varpi+O\left(|j|^{-\kappa}\right), \kappa=2, \varpi=1+\frac{b}{2} \sum_{k, l \in J} b_{k l} \xi_{l} .
$$

This verifies that (1.24) and (1.25) of Assumption $\mathbf{B}^{\star}$ hold true.

For a matrix $X$, by $X(k)$ denote the $k-$ th row of $X$. Then by (10.35), (10.36) and $(10.40)$, we get

$$
\begin{aligned}
& \left(S B^{-1}\right)(i)=\left(S B_{0}^{-1}\right)(i)+O\left(\frac{N}{L^{2}}\right) \\
= & \frac{b\left(\lambda_{i}+1\right)}{2 \sqrt{\lambda_{i}}}\left(\left(\begin{array}{ccc}
1 & \cdots & 1 \\
1 & \cdots & 1 \\
\vdots & \ddots & \vdots \\
1 & \cdots & 1 \\
\vdots & \vdots & \vdots
\end{array}\right) B_{0}^{-1}\right)(i)+O\left(\frac{N}{L^{2}}\right) \\
= & \frac{b\left(1+\lambda_{i}\right)}{2 \sqrt{\lambda_{i}}(a-b)(a+(N-1) b)}(-b(N-1)+a+(N-2) b, \cdots,-b(N-1)+a+(N-2) b)+O\left(\frac{N}{L^{2}}\right) \\
= & \frac{b\left(1+\lambda_{i}\right)}{2 \sqrt{\lambda_{i}}(a+(N-1) b)}(1, \cdots, 1)+O\left(\frac{N}{L^{2}}\right),
\end{aligned}
$$

where we have used $\sqrt{\lambda_{l}}=1+O\left(\frac{1}{L^{2}}\right)$ with $l \in J$. For any $k \in \mathbb{Z}^{N} \backslash\{0\}$, we assume $k_{1} \neq 0$ without loss of generality. Arbitrarily take an infinite dimensional integer vector $l=\left(l_{j} \in \mathbb{Z}: j \in \mathbb{Z}_{+}^{d} \backslash J\right)$ with $|l|=\sum_{j \in \mathbb{Z}_{+}^{d} \backslash J}\left|l_{j}\right| \leq 2$. Let $\left\langle l, \Omega^{0}(\xi)\right\rangle=\sum_{j \in \mathbb{Z}_{+}^{d} \backslash J} l_{j} \Omega_{j}^{0}(\xi)$. Then

$$
\left|\frac{d}{d \xi_{1}}\left(\left\langle k, \omega^{0}(\xi)\right\rangle+\left\langle l, \Omega^{0}(\xi)\right\rangle\right)\right|=\left|k_{1}+\sum_{j \in \mathbb{Z}_{+}^{d} \backslash J} l_{j} \frac{b^{2}\left(1+\lambda_{j}\right)}{2 \sqrt{\lambda_{j}}(a+(N-1) b)}\right|+O\left(\frac{N}{L^{2}}\right) .
$$

Note $\|j\|^{2}=\tau_{1}^{2} j_{1}^{2}+\cdots+\tau_{d} j_{d}^{2} \geq 1$. So

$$
\left|\sum_{j \in \mathbb{Z}_{+}^{d} \backslash J} l_{j} \frac{b\left(1+\lambda_{j}\right)}{2 \sqrt{\lambda_{j}}(a+(N-1) b)}\right| \leq \frac{1}{2} \sqrt{\frac{7}{2}} \frac{b}{a+(N-1) b}<\frac{b}{a+(N-1) b} .
$$


Assume $N \geq 2$. We have

$$
\begin{aligned}
\left|\frac{d}{d \xi_{1}}\left(\left\langle k, \omega^{0}(\xi)\right\rangle+\left\langle l, \Omega^{0}(\xi)\right\rangle\right)\right| & \geq 1-\frac{b}{a+(N-1) b}+O\left(\frac{N}{L^{2}}\right) \\
& \geq \frac{a+(N-2) b}{a+(N-1) b}+O\left(\frac{N}{L^{2}}\right) \\
& \geq C(N)>0, \text { for } L \gg N .
\end{aligned}
$$

This verifies condition (1.26) of Assumption $\mathbf{B}^{\star}$. Let $q=p>d / 2$ and

$$
D_{p}:=D_{p}\left(\varepsilon_{0}\right)=\left\{(x, y, \hat{z}, \bar{z}) \in \mathbb{C}^{N} /(2 \pi \mathbb{Z})^{N} \times \mathbb{Z}^{N} \times h_{p} \times h_{p}:|\mathfrak{I} x| \leq s_{0},|y| \leq \varepsilon_{0},\|\hat{z}\|_{p} \leq \varepsilon_{0}^{1 / 3},\|\overline{\hat{z}}\|_{p} \leq \varepsilon_{0}^{1 / 3}\right\} .
$$

By (10.26), 10.27, (10.28) and (10.34) we verify Assumption $\mathbf{C}$ and

$$
\left|\left\lfloor X_{R^{0}}\right\rceil\right|_{q, D_{p} \times \Pi} \leq C \varepsilon_{0},\left|\left\lfloor\partial_{\xi} X_{R^{0}}\right\rceil\right|_{q, D_{p} \times \Pi} \leq C \sqrt{\varepsilon_{0}} .
$$

It follows from (10.21), 10.25) and (10.27) that Assumption $\mathbf{D}$ holds true. Finally, Assumption $\mathbf{E}$ holds true clearly, since $B=0$. Using Theorem 1.2 we have

Theorem 10.5. Assume $\left(\frac{2 \pi}{T_{j}}: j \in \mathbb{Z}_{+}^{d}\right)$ is in $\tilde{\Theta}$. Around the neighborhood of $u=0$, gPC equation 10.1 has many (the initial value set of $\mathrm{N}$-dimensional positive Lebesgue measure) smooth solutions which are quasi-periodic in time, linear stable and of zero Lyapunov exponent. More exactly, there exists $\varepsilon_{0}^{*}=\varepsilon_{0}^{*}(N, \tau, J)>0$ depending on $N, \tau, J$ such that for any $0<\varepsilon_{0}<\varepsilon_{0}^{*}$ there is a subset $\breve{\Pi}$ of the initial value set $\Pi_{0}:=\left[\sqrt{\varepsilon_{0}}, 2 \sqrt{\varepsilon_{0}}\right]^{N}$ with

$$
\operatorname{Leb} \breve{\Pi}=\left(\operatorname{Leb} \Pi_{0}\right)\left(1-C \frac{1}{\left|\log \varepsilon_{0}\right|}\right)
$$

and for any $\xi=\left(\xi_{l}: l=1, \ldots, N\right) \in \breve{\Pi}, g P C$ equation has a quasi-periodic solution $u(t, x)$ of frequency $\omega \in \mathbb{R}^{N}$ in time $t$

$$
u(t, x)=\sum_{k \in \mathbb{Z}^{N}, j \in \mathbb{Z} \backslash\{0\}} \hat{u}(k, j) e^{\mathbf{i}(k, \omega)} \phi_{j}(x)
$$

with

$$
\phi_{n}(x)=\sin n_{1} \tau_{1} x_{1} \cdots \sin n_{d} \tau_{d} x_{d}, \forall n=\left(n_{1}, \ldots, n_{d}\right) \in \mathbb{Z}_{+}^{d},
$$

satisfying

$$
\begin{gathered}
\left|\omega-\omega_{0}\right| \leq C \sqrt{\varepsilon_{0}}, \omega \in \mathbb{R}^{N}, \omega_{0}=\left(\sqrt{\frac{\left\|j_{l}\right\|^{2}}{1+\|\left. j_{l}\right|^{2}}}: j_{l} \in J\right) \in \mathbb{R}^{N}, \\
\left|\hat{u}\left(e_{l}, j_{l}\right)-\xi_{l}\right|<C \varepsilon_{0}^{1 / 3}, e_{l}-l^{\text {th }} \text { unit vector of } \mathbb{Z}^{N}, j_{l} \in J, l=1, \ldots, N,
\end{gathered}
$$

and

$$
\sum_{(k, j) \notin \mathscr{S}} \mid \hat{u}\left(k,\left.j\right|^{2} e^{|k| s_{0}+2 a|j|}|j|^{2 p}<C \varepsilon_{0}^{1 / 3}, \quad \mathscr{S}=\left(e_{l}, j_{l}\right): l=1, \ldots, N,\right.
$$

where some constants $s_{0}>0, a>0$ and $p>d / 2$.

Remark 13. Theorem 1.2 applies to more general PC equation:

$$
\left\{\begin{array}{l}
u_{t t}-\Delta u-\Delta u_{t t}+F(u, \Delta u)=0, x \in \Omega \subset \mathbb{R}^{d}, \\
\left.u\right|_{\partial \Omega}=0
\end{array}\right.
$$

where $F(u)=\sum_{j+j \geq 3} c_{i j} u^{i}(\Delta u)^{j}$ is an analytic function of $u$ and $\Delta u$ with $c_{i j} \in \mathbb{R}$. 


\section{Final Remark on global solutions to BBM and gPC}

Up to now there have been a lot of works on the existence and long-time asymptotic behavior as well as traveling solutions for BBM and gPC where the spatial variable is in the whole space $\mathbb{R}^{d}$. See [47, 48, 49, 50, 51] for BBM and [52, 53, 54, 55] for gPC and the references therein. According to our knowledge, there is not any results on the existence of solutions, let alone long-time behavior of solutions, for BBM and gPC when the spatial variable $x$ is in some compact space, $\mathbb{T}^{d}$, say.

By Theorems 9.5 and 10.5, 1-dimensional BBM and $d$-dimensional gPC equations subject to typical periodic boundary conditions (the spatial variable in compact space) have many quasi-periodic solutions with initial values of positive finite dimensional Lebesgue measure. These quasi-periodic solutions are of course global and of recurrent property. As done in [56], Those solutions whose initial date close to any quasi-periodic solution are almost global, that is, assuming $u_{0}(t, x)$ with initial datum $u_{0}(0, x)$ is a quasi-periodic solution for BBM or gPC equation subject the typical boundary conditions, then any solution $u(t, x)$ with initial value satisfying $\left\|u(0, x)-u_{0}(0, x)\right\|_{p}<\delta$ with any $0<\delta \ll 1$ obeys that the solution $u(t, x)$ exists for time $|t|<L \delta^{-1}$ and

$$
\left\|u(t, \cdot)-u_{0}(t, \cdot)\right\|_{p} \leq C \delta, \quad \forall|t|<\delta^{-1} .
$$

\section{Appendices}

Let $\tilde{p}, \tilde{q}>d / 2$. For a linear operator $L: h_{\tilde{p}} \rightarrow h_{\tilde{q}}$, denote by $L_{i j}$ 's the matrix elements of $L$. Given an index set $I \subset \mathbb{Z}^{d}$. Partition $L$ as follows

$$
L=\left(\begin{array}{ll}
L^{(11)} & L^{(12)} \\
L^{(21)} & L^{(22)}
\end{array}\right)
$$

where

$$
\begin{gathered}
L^{(11)}=\left(L_{i j}: i \in I, j \in I\right), L^{(12)}=\left(L_{i j}: i \in I, j \in \mathbb{Z}^{d} \backslash I\right), \\
L^{(21)}=\left(L_{i j}: i \in \mathbb{Z}^{d} \backslash I, j \in I\right), L^{(22)}=\left(L_{i j}: i \in \mathbb{Z}^{d} \backslash I, j \in \mathbb{Z}^{d} \backslash I\right) .
\end{gathered}
$$

Expand $L^{(i j)}(i, j \in\{1,2\})$ to $\tilde{L}^{(i j)}$ as follows

$$
\tilde{L}^{(11)}=\left(\begin{array}{cc}
L^{(11)} & 0 \\
0 & 0
\end{array}\right), \tilde{L}^{(12)}=\left(\begin{array}{cc}
0 & L^{(12)} \\
0 & 0
\end{array}\right), \tilde{L}^{(21)}=\left(\begin{array}{cc}
L^{(21)} & 0 \\
0 & 0
\end{array}\right), \tilde{L}^{(22)}=\left(\begin{array}{cc}
0 & 0 \\
0 & L^{(22)}
\end{array}\right) .
$$

Define

$$
\left\|L^{(i j)}\right\|_{h_{\tilde{p}}^{i} \rightarrow h_{\tilde{q}}^{j}}=\left\|\tilde{L}^{(i j)}\right\|_{h_{\tilde{p}} \rightarrow h_{\tilde{q}}}
$$

According to the partition of $L$, split the space $h_{\tilde{p}}$ :

$$
h_{\tilde{p}}=h_{\tilde{p}}^{1} \oplus h_{\tilde{p}}^{2},
$$

where $h_{\tilde{p}}^{1}=\left\{\left(z_{j} \in \mathbb{C}: j \in I\right)\right\}$ and $h_{\tilde{p}}^{2}=\left\{\left(z_{j} \in \mathbb{C}: j \in \mathbb{Z}^{d} \backslash I\right)\right\}$. And define

$$
\|z\|_{h_{\tilde{p}}^{1}}^{2}=\sum_{j \in I}|j|^{2 \tilde{p}}\left|z_{j}\right|^{2}, \quad z \in h_{\tilde{p}}^{1}
$$

and

$$
\|\left. z\right|_{h_{\tilde{p}}^{2}} ^{2}=\sum_{j \in \mathbb{Z}^{d} \backslash I}|j|^{2 \tilde{p}}\left|z_{j}\right|^{2}, \quad z \in h_{\tilde{p}}^{2} .
$$

Lemma 12.1. For any $\tilde{p}, \tilde{q} \in\{p, q=p+\kappa\}$ and any $i, j \in\{1,2\}$, we have

$$
\left\|L^{(i j)}\right\|_{h_{\tilde{p}}^{i} \rightarrow h_{\tilde{q}}^{j}} \leq\left.|| L\right|_{h_{\tilde{p}} \rightarrow h_{\tilde{q}}} .
$$


Proof. The proof can be found in P. 104, [45].

Lemma 12.2. Assuming that $X$ is self-adjoint in $\ell_{2}$ and assuming that $\operatorname{Dim} X<\infty$, we have

$$
\|X\|_{\ell_{2} \rightarrow \ell_{2}} \leq\|X\|_{h_{p} \rightarrow h_{p}}, \forall p>0
$$

Proof. Let $\lambda$ be any eigenvalue of $X$ and $x_{0}$ be the eigenvector with the eigenvalue $\lambda$. Since $X$ is self-adjoint, $\|X\|_{\ell_{2} \rightarrow \ell_{2}}=\sup \{|\lambda|\}$. Write $X=\left(X_{i j}: i, j \in \Xi\right)$. Let $I=\operatorname{diag}\left(|j|^{p}: j \in \Xi\right)$. Then

$$
\|X\|_{h_{p} \rightarrow h_{p}}=\sup _{x \neq 0} \frac{\|X x\|_{h_{p}}}{\|x\|_{h_{p}}}=\sup _{x \neq 0} \frac{\|I X x\|_{\ell_{2}}}{\|I x\|_{\ell_{2}}} \geq \frac{\left\|I X x_{0}\right\|_{\ell_{2}}}{\left\|I x_{0}\right\|_{\ell_{2}}}=\frac{\left\|I \lambda x_{0}\right\|_{\ell_{2}}}{\left\|I x_{0}\right\|_{\ell_{2}}}=|\lambda| .
$$

Thus

$$
\|X\|_{h_{p} \rightarrow h_{p}} \geq \sup \{|\lambda|\}=\|X\|_{\ell_{2} \rightarrow \ell_{2}}
$$

Lemma 12.3. Assume $\lfloor X\rceil$ and $\lfloor Y\rceil$ are two bounded operator from $h_{\tilde{p}}$ to $h_{\tilde{q}}$ where $\tilde{p}, \tilde{q} \in\{p, q, 0\}$. Then

$$
\|\lfloor X+Y\rceil\|_{h_{\tilde{p}} \rightarrow h_{\tilde{q}}} \leq\|\lfloor X\rceil\|_{h_{\tilde{p}} \rightarrow h_{\tilde{q}}}+\|\lfloor Y\rceil\|_{h_{\tilde{p}} \rightarrow h_{\tilde{q}}}
$$

and

$$
\|\lfloor X Y\rceil\|_{h_{\tilde{p}} \rightarrow h_{\tilde{p}}} \leq\|\lfloor X\rceil\|_{h_{\tilde{p}} \rightarrow h_{\tilde{p}}}\|\lfloor Y\rceil\|_{h_{\tilde{p}} \rightarrow h_{\tilde{p}}}
$$

Proof. The proof is easily verified by the definitions of $\|\cdot\|_{h_{\tilde{p}} \rightarrow h_{\tilde{q}}}$ and $\lfloor\cdot\rceil$. We omit it.

Lemma 12.4. For a finite dimensional matrix $X=\left(X_{i j}: i, j \in \mathbb{Z}^{d},|i|,|j| \leq \Gamma\right)$, where $\Gamma$ is a fixed constant, then

$$
\|\lfloor X\rceil\|_{h_{\tilde{p}} \rightarrow h_{\tilde{q}}} \leq \Gamma^{d / 2}\|X\|_{h_{\tilde{p}} \rightarrow h_{\tilde{q}}},
$$

where $h_{\tilde{p}}$ is a space of finite dimensional vectors:

$$
h_{\tilde{p}}=\left\{z=\left(z_{j} \in \mathbb{C}: j \in \mathbb{Z}^{d},|j| \leq \Gamma\right)\right\}
$$

with

$$
\|z\|_{h_{\tilde{p}}}^{2}=\sum_{|j| \leq \Gamma}|j|^{2 \tilde{p}}\left|z_{j}\right|^{2}
$$

Proof. Introducing a weight $w_{i j}=|i|^{\tilde{p}}|j|^{-\tilde{q}}$. Let $\tilde{X}_{i j}=w_{i j} X_{i j}$. Then

$$
\|\tilde{X}\|_{h_{0} \rightarrow h_{0}}=\|X\|_{h_{\tilde{p}} \rightarrow h_{\tilde{q}}},\|\lfloor\tilde{X}\rceil\|_{h_{0} \rightarrow h_{0}}=\|\lfloor X\rceil\|_{h_{\tilde{p}} \rightarrow h_{\tilde{q}}} .
$$

Let $\delta=\|\tilde{X}\|_{h_{0} \rightarrow h_{0}}$. Then

$$
\max _{|i| \leq \Gamma} \sum_{|j| \leq \Gamma}\left|\tilde{X}_{i j}\right|^{2} \leq \delta^{2}, \max _{|j| \leq \Gamma} \sum_{|i| \leq \Gamma}\left|\tilde{X}_{i j}\right|^{2} \leq \delta^{2} .
$$

For any $u=\left(u_{j}:|j| \leq \Gamma\right)$ with $\|u\|_{h_{0}}=1$,

$$
\begin{aligned}
\| \mid X u\rceil \|_{h_{0} \rightarrow h_{0}}^{2} & =\sum_{|i| \leq \Gamma}\left|\sum_{|j| \leq \Gamma}\right| \tilde{X}_{i k}\left|u_{j}\right|^{2} \\
& \leq \sum_{|i| \leq \Gamma}\left(\sum_{|j| \leq \Gamma}\left|\tilde{X}_{i j}\right|^{2}\right)\left(\sum_{|j| \leq \Gamma}\left|u_{j}\right|^{2}\right) \\
& \leq \sum_{|i| \leq \Gamma} \delta^{2} \\
& \leq \Gamma^{d} \delta^{2} .
\end{aligned}
$$

This completes the proof. 


\section{Acknowledgements}

I am very grateful to the referee for the invaluable suggestions. This article is a revised and updated version, replacing a $d$-dimensional generalized BBM equation by a $d$-dimensional generalized PC equation, of the 2017August version "KAM theorem with normal frequencies clustering at zero for some shallow water equations". The author began to conceive this article when visiting the Mittag-Leffler Institute in 2010 invited by Professor H. Eliasson and Professor J.-C. Yoccoz. In 2015, the author was invited by Professor S. Kuksin to report the early version of this article at the Euler Institute. In 2016, the author was invited by Professor F. Meng to visit Qufu Normal University where the author made some revisions. Then the author had a beneficial discussion with Professor J. Liu. In this long writing process, the author was also encouraged by Professor W. Craig, Professor D. Bambusi, and Professor B. Grebert. In addition, Professor M. Gao and Professor J. Li helped the author edit part of the manuscript with latex. The author would like to express his sincere gratitude to all the professors mentioned above. The author is also thankful to Dr. K. Zhang for her help in computing the normal form of BBM and to Professor H. Cong and Dr. Y. Shi for their reading the manuscript.

\section{References}

[1] Avrin, J., Goldstein, J.A., Global existence for the Benjamin-Bona-Mahony equation in arbitrary dimensions, Nonlinear Anal., 9:8 (1985) 861-865.

[2] Baldi, P., Berti, M. and Montalto, R., KAM for quasi-linear and fully nonlinear forced perturbations of Airy equation, Math. Ann., 359:1-2 (2014) 471-536.

[3] Baldi, P., Berti, M., Haus, E. and Montalto, R., Time quasi-periodic gravity water waves in finite depth, (2017), arXiv:1708.01517

[4] Baldi, P., Berti, M. and Montalto, R., KAM for autonomous quasi-linear perturbations of KdV, Ann. Inst. H. Poincaré Anal. Non Linéaire, 33:6 (2016) 1589-1638.

[5] Bambusi, D., Grébert, B., Birkhoff normal form for partial differential equations with tame modulus, Duke Math. J., 135:3 (2006) 507-567.

[6] Benjamin, T. B., Bona, J. L. and Mahony, J. J., Model equations for long waves in nonlinear dispersive systems, Philosophical Transactions of the Royal Society of London. Series A, Mathematical and Physical Sciences, 272:1220 (1972) 47-78.

[7] Berti, M., Biasco, L. and Procesi, M., KAM theory for the Hamiltonian derivative wave equation, Annales Scientifiques de l'Ecole Normale Superieure. Societe Mathematique de France, 46:2 (2013) 301-373.

[8] Berti, M, Montalto, R. Quasi-periodic standing wave solutions of gravity-capillary water waves, (2016) arXiv:1602.02411

[9] Piotr, B., Long time behavior of solutions of the generalized Benjamin-Bona-Mahony equation in two space dimensions. Differential Integral Equations 5: 4 (1992) 891-901.

[10] Bogolubsky,I.L., Some examples of inelastic soliton interaction, Computer Physics Communication, 13 (3), (1977) 149-155.

[11] Bourgain, J., Construction of quasi-periodic solutions for Hamiltonian perturbations of linear equations and application to nonlinear PDE, Int. Math. Research Notices, 11 (1994) 475-497.

[12] Bourgain, J., On Melnikov's persistency problem, Math. Research Letters, 4:4 (1997) 445-458. 
[13] Bourgain, J., Quasi-periodic solutions of Hamiltonian perturbations for 2D linear Schrödinger equation, Ann. Math., 148 (1998) 363-439.

[14] Bourgain, J., Periodic solutions of nonlinear wave equations, Harmonic analysis and partial equations, Chicago Univ. Press, 1999, pp.69-97.

[15] Bourgain, J., Recent progress on quasi-periodic lattice Schrodinger operators and Hamiltonian PDEs, Russian Math. Surveys, 59 :2 (2004) 231-46.

[16] Bourgain, J., Green function estimates for lattice Schrödinger operators and applications, Annals of Math. Studies, Princeton University Press, 2005.

[17] Clarkson, P. A., Le Vaque, R.J. and Saxton,R., Solitary wave interactions in elastic rods, Studies in Applied Mathematics 75, (1986) 95-121.

[18] Chree, C., Longitudinal vibrations of a circular bar, Quarterly Journal of Mathematics 21, (1886) 287-288.

[19] Craig, W., Wayne, C.E., Newton's method and periodic solutions of nonlinear wave equations, Commun. Pure. Appl. Math., 46:11 (1993) 1409-1498.

[20] Craig, W., Problemes de petits diviseurs dans les equations aux derivees partielles, Panoramas et Syntheses 9, Soc. Math. France, 2000

[21] Eliasson L H. Perturbations of stable invariant tori for Hamiltonian systems, Annali della Scuola Normale Superiore di Pisa-Classe di Scienze, 15: 1(1988) 115-147.

[22] Eliasson, L.H., Kuksin, S.B., KAM for the nonlinear Schrödinger equation, Ann. Math., 172 (2010) 371-435.

[23] Eliasson L H, Grébert B, Kuksin S B. KAM for the nonlinear beam equation, Geometric and Functional Analysis, 26:6(2016) 1588-1715.

[24] Feola, R., Procesi, M., Quasi-periodic solutions for fully nonlinear forced reversible Schrödinger equations, J. Differential Equations, 259:7 (2015) 3389-3447.

[25] Halmos, P.R., Sunder, V. S., Bounded integral operators on $L^{2}$ space, Springer-Verlag, Berlin Heidelberg New York, 1978.

[26] Goldstein, J.A., Wichnoski, B.J., On the Benjamin-Bona-Mahony equation in higher dimensions, Nonlinear Analysis, 4:4 (1980), 665-675.

[27] Kappeler, T., Pöschel, J., KdV and KAM, A Series of Modern Surveys in Mathematics, 45, Springer-Verlag, Berlin Heidelberg, 2003.

[28] Kato, T., Perturbation theory for linear operators, Corrected printing of the second edition Springer-Verlag, Berlin Heidelberg New York, 1980.

[29] Kuksin, S.B., Perturbations of quasiperiodic solutions of infinite-dimensional Hamiltonian systems, Izv. Akad. Nauk SSSR Ser. Mat. 52 (1988) 41-63, Engl. Transl. in Math. USSR Izv. 32:1 (1989).

[30] Kuksin, S.B., Nearly integrable infinite-dimensional Hamiltonian systems, Lecture Notes in Math., 1556, Springer-Verlag, New York, 1993.

[31] Kuksin, S.B., Analysis of Hamiltonian PDEs, Oxford University Press, 2000.

[32] Kuksin, S.B., Fifteen Years of KAM for PDE, Trans. Amer. Math. Soc., 212:2 (2004) 237-258. 
[33] Kuksin, S.B., Pöschel, J., Invariant Cantor manifolds of quasiperiodic oscillations for a nonlinear Schrödinger equation, Ann. Math., 143:1 (1996) 149-179.

[34] Lin, Zh., Zeng, Ch., Instability, index theorem, and exponential trichotomy for Linear Hamiltonian PDEs, arXiv:1703.04016, 2017.

[35] Liu, J., Yuan, X., A KAM Theorem for Hamiltonian Partial Differential Equations with Unbounded Perturbations, Commun. Math. Phys., 307 (2011) 629-673.

[36] Liu, J., Yuan, X., Spectrum for quantum Duffing oscillator and small-divisor equation with large variable coefficient. Commun. Pure. Appl. Math., 63:9 (2010) 1145-1172.

[37] Melnikov, V. K., On some cases of the conservation of conditionally periodic motions under a small change of the Hamiltonian function, Soviet Math. Dokl., 6 (1965) 1592-1596.

[38] Parker, A., On exact solutions of the regularized long-wave equation: a direct approach to partially integrable equations. I. Solitary wave and solitons, J. Math. Phys. 36, (1995) 3498. doi.org/10.1063/1.530976

[39] Parand, K. and Rad, J., Some solitary wave solutions of generalized PochhammerCChree equation via Expfunction method, World Academy of Science, Engineering and Technology 43, (2010) 423.

[40] Pochhammer, L., Ueber die Fortpflanzungsgeschwindigkeiten kleiner Schwingungen in einem unbegrenzten isotropen Kreiscylinder, Journal für die reine und angewandte Mathematik, 81 (1876): 324-336.

[41] Pöschel, J., A KAM-theorem for some nonlinear partial differential equations, Ann. Scuola Norm. Sup. Pisa, Cl. Sci., Seire IV, 23:1 (1996) 119-148.

[42] Pöschel, J., Quasi-periodic solutions for nonlinear wave equation, Commun. Math. Helvetici, 71:1 (1996) 269-296.

[43] Reed, M., Simon, B., Methods of modern mathematical physics, 1, Functional analysis, Academic Press, San Diego, New York, 1980.

[44] Wayne, C. E., Periodic and quasi-periodic solutions of nonlinear wave equations via KAM theory, Commun. Math. Phys., 127:3 (1990) 479-528.

[45] Yuan, X., A KAM theorem with applications to partial differential equations of higher dimensions, Comm. Math. Physics, 275:1 (2007) 97-137.

[46] Zhang, J., Gao, M. and Yuan, X., KAM tori for reversible partial differential equations, Nonlinearity 24:4 (2011) 1189-1228.

[47] Naher, H. and and Abdullah, F. A., The Modified Benjamin-Bona-Mahony equation via the extended generalized Riccati equation mapping method, Applied Mathematical Sciences, 6:111, (2012), 5495 - 5512

[48] Biler, P., Long time behavior of solutions of the generalized Benjamin-Bona-Mahony equation in two space dimensions, Differential and Integral Equations, 5: 4, (1992) 891-901.

[49] Wang, M., Long time dynamics for a damped Benjamin-Bona-Mahony equation in low regularity spaces,Nonlinear Analysis, 105 (2014) 134-144

[50] Albert., J., On the Decay of Solutions of the Generalized Benjamin-Bona-Mahony Equation, J. of mathematical analysis and applications, 141, (1989) 527-537

[51] Fang, Sh. and Guo, B., The decay rates of solutions of generalized Benjamin-Bona-Mahony equations in multi-dimensions, Nonlinear Analysis 69, (2008) 2230-2235 
[52] Triki, H., Benlalli, A., and Wazwaz, A. M., Exact solutions of the generalized Pochhammer-Chree equation with sixth-order dispersion. Rom. J. Phys, 60, (2015) 935-951.

[53] Shawagfeh, N., and D. Kaya, Series solution to the Pochhammer-Chreeequation and comparison with exact solutions, Computers and Mathematics with Applications 47:12 (2004) 1915-1920

[54] Zhao, Y. and Zhang, W., Qualitative analysis and travelling wave solutions for the generalized PochhammerCChree equation with a dissipation term, Studies in Applied Mathematics, 121:4, (2008) 369-394

[55] Xu, R. and Liu, Y. Global existence and blow-up of solutions for generalized Pochhammer-Chree equations, Acta Mathematica Scientia 30: 5 (2010) 1793-1807.

[56] Cong, H., Liu, J. and Yuan, X., Stability of KAM tori for nonlinear Schrödinger equation, 239: 1134 (2016), American Mathematical Society. 